\title{
Kyrgyz Republic: Statistical Appendix
}

This Statistical Appendix for the Kyrgyz Republic was prepared by a staff team of the International Monetary Fund as background documentation for the periodic consultation with the member country. It is based on the information available at the time it was completed on November 5, 2004. The views expressed in this document are those of the staff team and do not necessarily reflect the views of the government of the Kyrgyz Republic or the Executive Board of the IMF.

The policy of publication of staff reports and other documents by the IMF allows for the deletion of market-sensitive information.

To assist the IMF in evaluating the publication policy, reader comments are invited and may be sent by e-mail to publicationpolicy@imf.org.

Copies of this report are available to the public from

International Monetary Fund $\bullet$ Publication Services

700 19th Street, N.W. • Washington, D.C. 20431

Telephone: (202) 6237430 • Telefax: (202) 6237201

E-mail: publications@imf.org • Internet: http://www.imf.org

Price: $\$ 15.00$ a copy

\section{International Monetary Fund Washington, D.C.}





\section{INTERNATIONAL MONETARY FUND}

\section{KYRGYZ REPUBLIC}

\section{Statistical Appendix}

Prepared by a staff team consisting of Tapio Saavalainen (head), Brad McDonald,

Bakyt Dubashev, Nataliya Mylenko, Malina Savova (all MCD),

Kentaro Ogata (FAD), and Shaun Roache (PDR)

Approved by Middle East and Central Asia Department

November 5, 2004

Contents

Page

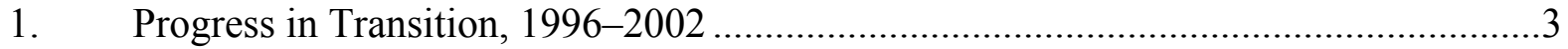

2. Value Added in the Main Production Sectors, 1995-2004 ........................................

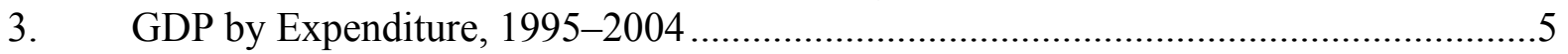

4. Industrial Production by Sectors, 1998-2004 ...............................................................

5. $\quad$ Output of Selected Industrial and Manufacturing Products, 1995-2004...................... 7

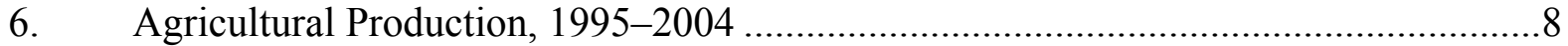

7. Agricultural and Animal Production by Farm Type, 2001-2003 ……….....................

8. Yields of Major Agricultural Commodities, 1995-2003 ……….................................10

9. Consumer and Producer Prices, 1998-2004 …….................................................11

10. Energy Sector Producer Prices, 1995-2004 ………................................................12

11. Nominal and Real Wages, 1995-2004 .................................................................13

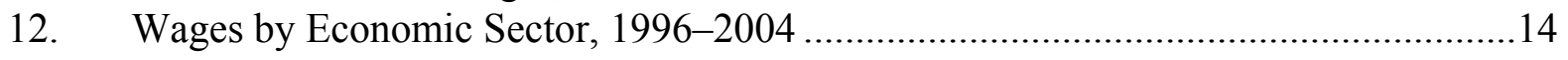

13. Labor Market, 1995-2003 ……………………...........................................15

14. Employment by Sector, 1995-2003 ………………………….............................16

15. Privatization by Sector and Type of Property, 1995-2004..........................................17

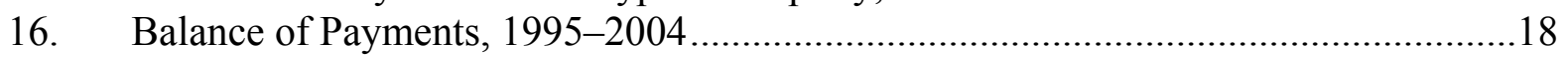

17. Exports of Goods by Product Category, 1995-2004 …………………….................19

18. Imports of Goods by Product Category, 1995-2004 ……………..........................2

19. Exports of Goods to CIS and Non-CIS Countries, 1995-2004 …….......................21

20. Imports of Goods from CIS and Non-CIS Countries, 1995-2004 ............................22

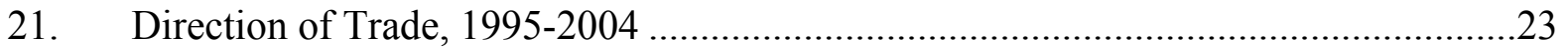

22. Production, Imports, and Exports of Energy Products, 1995-2004 ...........................24

23. Foreign Direct Investment by Country of Origin, 1995-2003 ..................................25

24. External Public Debt and Debt Service, 1995-2004 ................................................26

25a. State Government Finances (in millions of soms), 1995-2003 ………………..........2

25b. State Government Finances (in percent of GDP), 1995-2003 .................................28

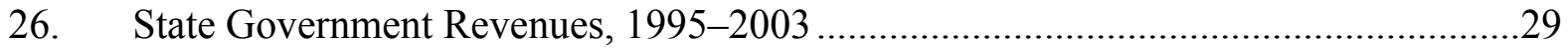


27. State Government Expenditure by Functional Classification, 1995-2003 _................30

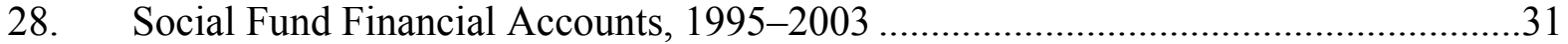

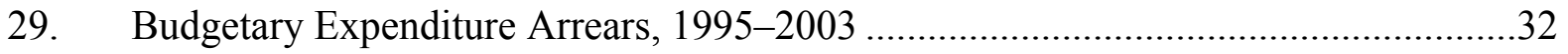

30. State Government Wage Bill by Sector, 1999-2003 …..........................................33

31. National Bank of Kyrgyz Republic Accounts, 1995-2004 …..................................34

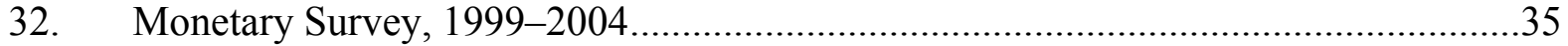

33. Key Central Bank Interest Rates, 1998-2004 ......................................................36

34. Interest Rates Developments, 1998-2004 ........................................................37

35. Foreign Exchange Auctions and Interbank Market, 1999-2004 ..............................38

36. Stock of Outstanding Government Securities, 1998-2004 .....................................39

37. Composition of Credits and Deposits to the Economy by Sectors,

Maturity, Currency and Bank, 1998-2004 ..........................................................40

38. Indicators of the Banking System's Financial Strength, 1999-2004.........................41

39. Banks with Licenses Revoked during 1996-2004 ..............................................42 
Table 1. Kyrgyz Republic: Progress in Transition, 1996-2002 1/

\begin{tabular}{|c|c|c|c|c|c|c|c|}
\hline & 1996 & 1997 & 1998 & 1999 & 2000 & 2001 & 2002 \\
\hline Private sector share of GDP (percent) 2/ & 40 & 50 & 60 & 60 & 60 & 60 & 60 \\
\hline \multicolumn{8}{|l|}{ Enterprises 3/ } \\
\hline Large-scale privatization & 4 & 3 & 3 & 3 & 3 & 3 & 3 \\
\hline Small-scale privatization & 4 & 4 & 4 & 4 & 4 & 4 & 4 \\
\hline Enterprise restructuring & 2 & 2 & 2 & 2 & 2 & 2 & 2 \\
\hline \multicolumn{8}{|l|}{ Markets and trade 4/ } \\
\hline Price liberalization & 3 & 3 & 3 & 3 & 3 & 3 & $4+$ \\
\hline Trade and foreign exchange system & 4 & 4 & 4 & 4 & 4 & 4 & $4+$ \\
\hline Competition policy & 2 & 2 & 2 & 2 & 2 & 2 & 2 \\
\hline \multicolumn{8}{|l|}{ Financial institutions 5/ } \\
\hline Banking reform and interest ratio liberalization & 2 & 2 & $3-$ & $3-$ & $2+$ & $2+$ & $2+$ \\
\hline Securities markets and non-bank financial institutions & 2 & 2 & 2 & 2 & 2 & 3 & 2 \\
\hline Infrastructure reform $6 /$ & $1+$ & $1+$ & $1+$ & $1+$ & $1+$ & $1+$ & $1+$ \\
\hline
\end{tabular}

Source: EBRD Transition Report.

1/ Based on the transition indicators produced by the EBRD, Table 2.1, 2002 Transition Report.

2/ EBRD mid-year estimate.

3/ For enterprises, in general a "1" ranking signals little private ownership or progress while a "4+" signals standards and performance typical of advanced industrial economics.

4/ For markets and trade, a "1" ranking signals extreme controls, while "4+" is typical of standards and performanceof advanced industrial countries.

5/ For financial institutes, a "1" ranking signals little progress, while "4+" is typical of standards and performance of advanced industrial countries.

6/ This rating is computed as an average of the reform process in telecommunications, electric power, water and waste water, roads and railways. 


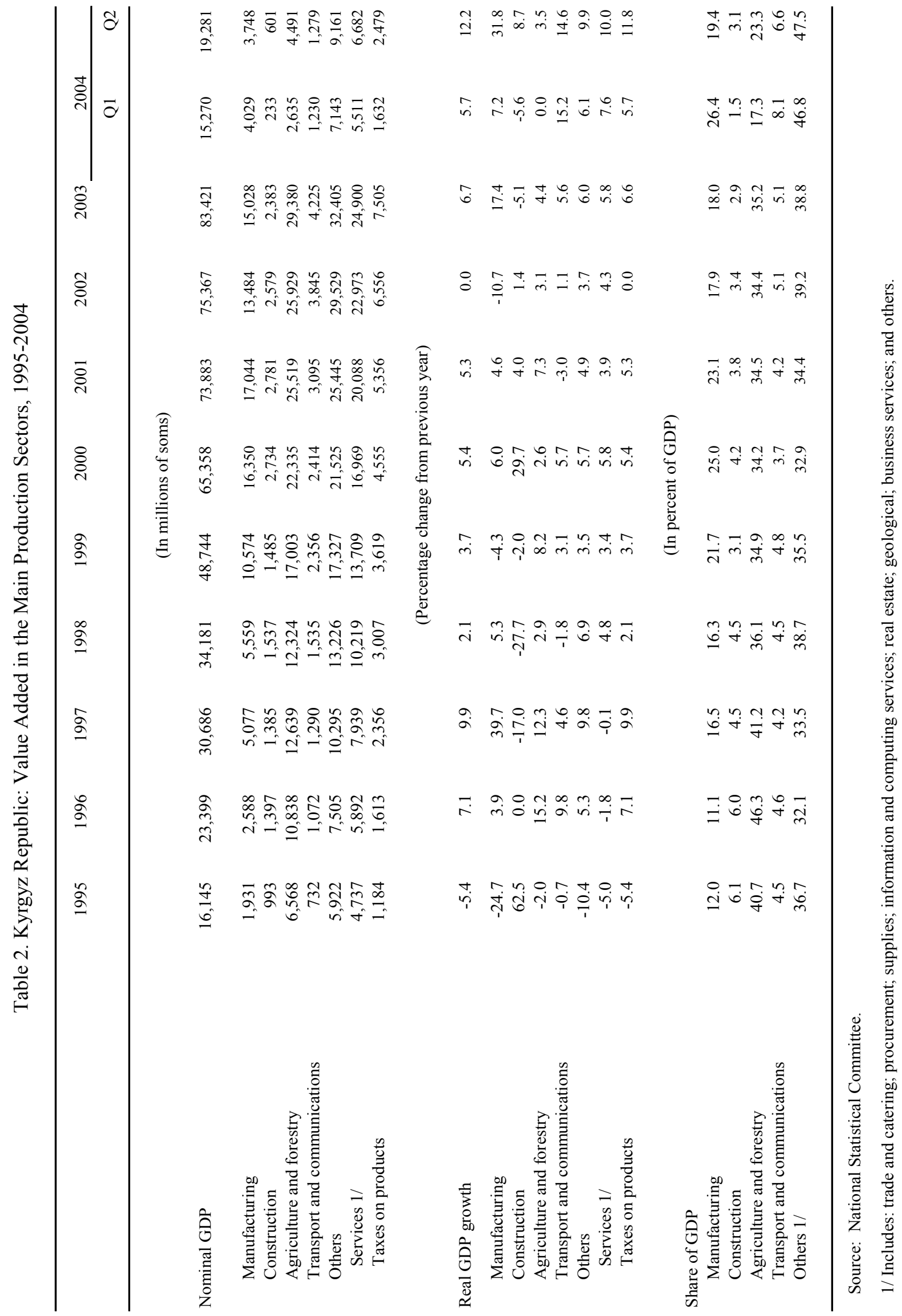




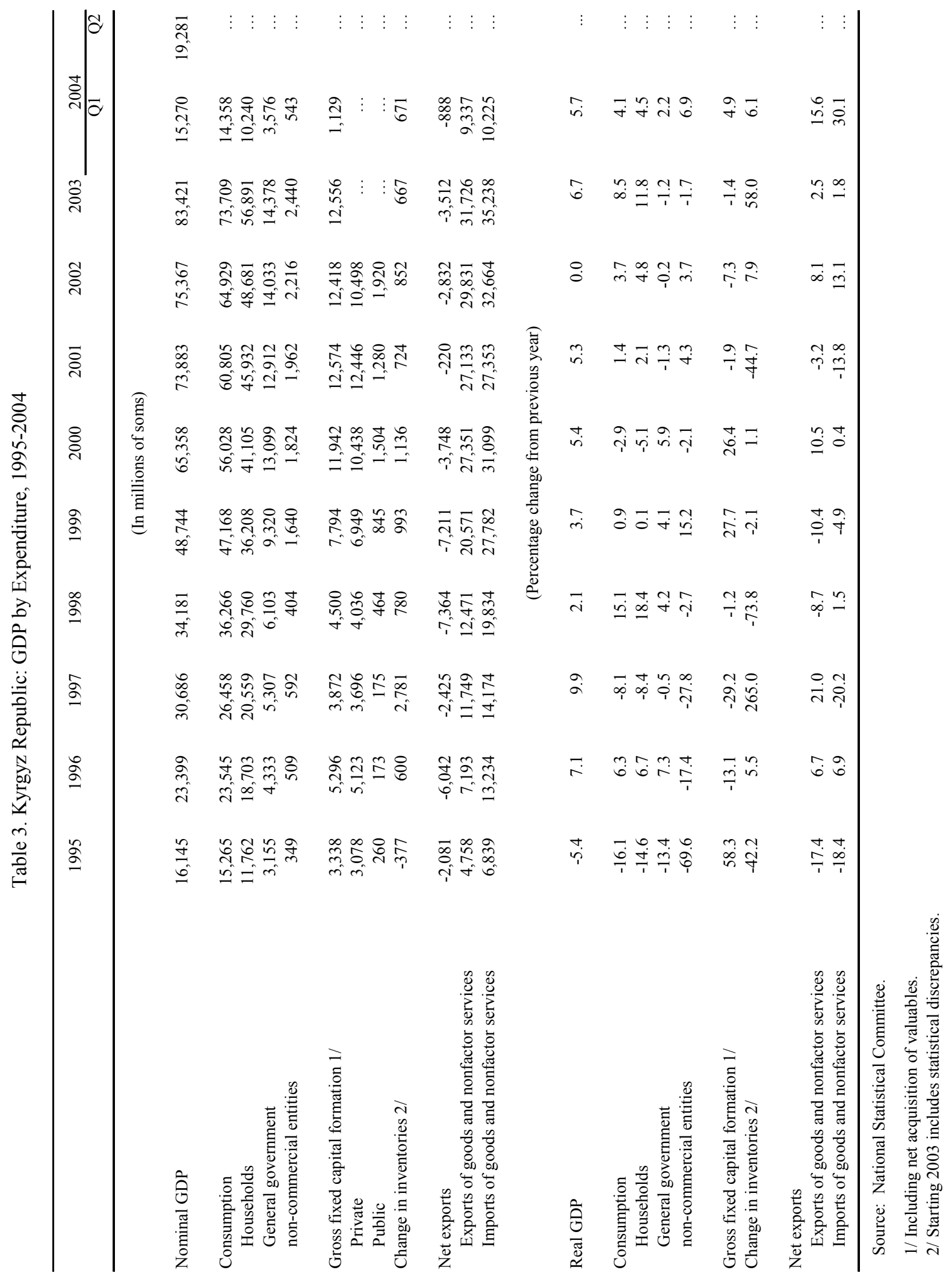




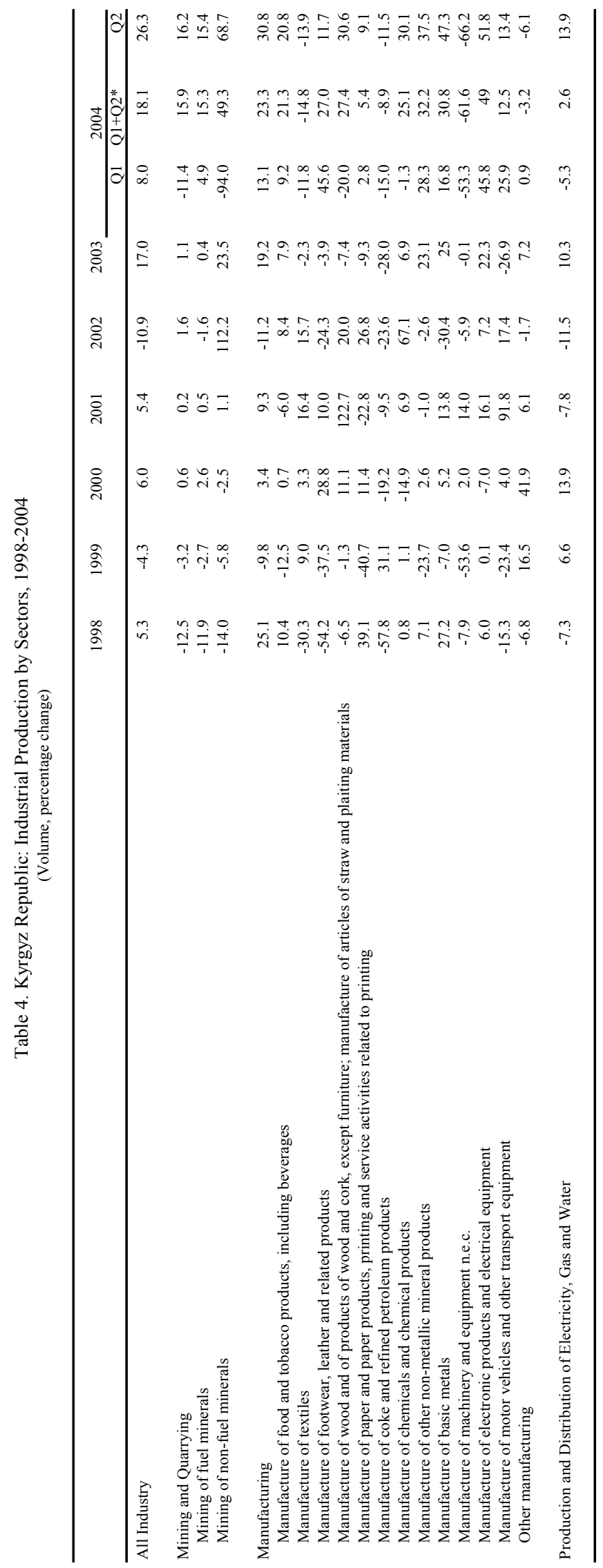




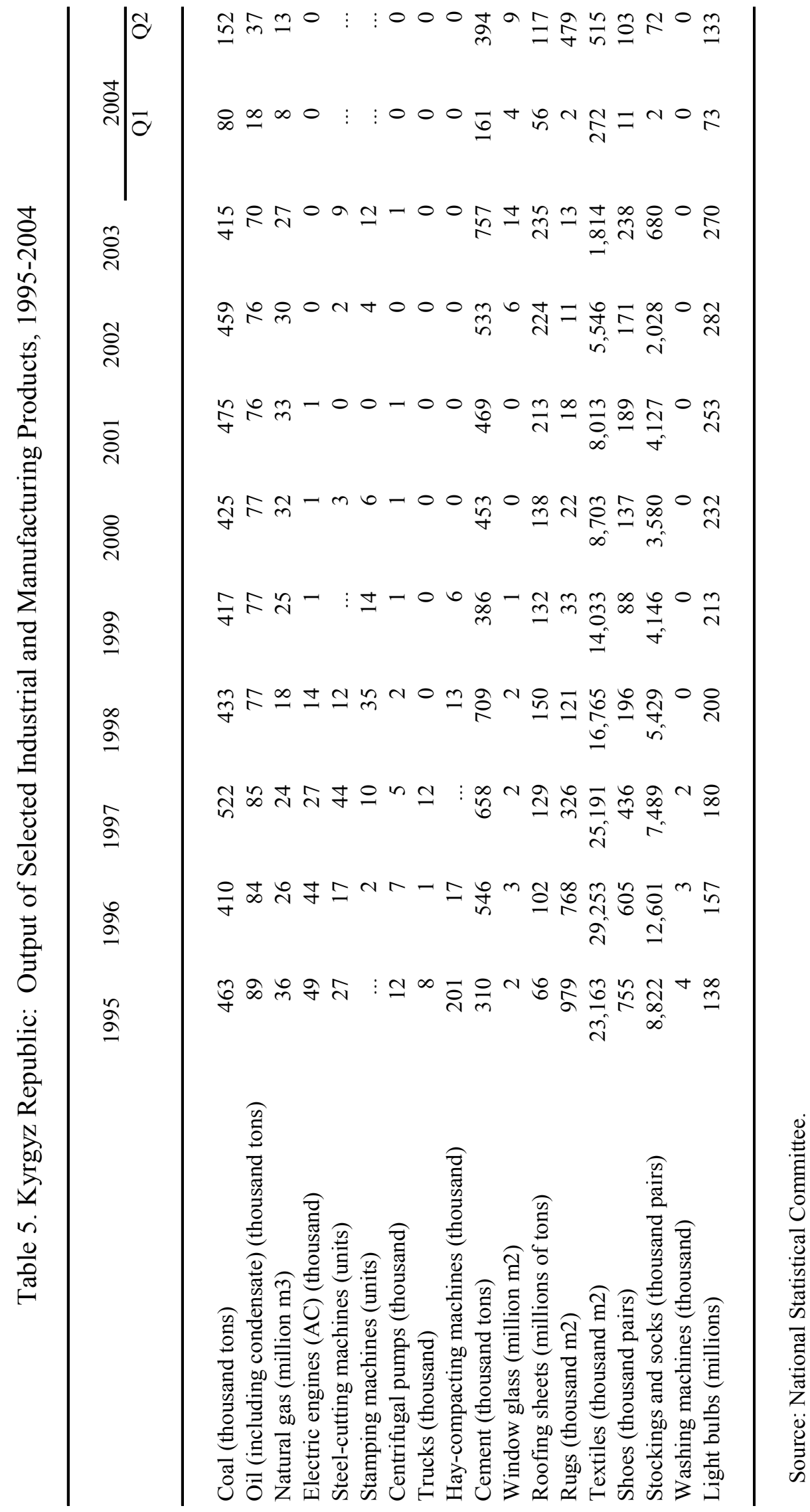


Table 6. Kyrgyz Republic: Agricultural Production, 1995-2004

\begin{tabular}{|c|c|c|c|c|c|c|c|c|c|c|c|}
\hline & \multirow{2}{*}{1995} & \multirow{2}{*}{1996} & \multirow{2}{*}{1997} & \multirow{2}{*}{1998} & \multirow{2}{*}{1999} & \multirow{2}{*}{2000} & \multirow{2}{*}{2001} & \multirow{2}{*}{2002} & \multirow{2}{*}{2003} & \multicolumn{2}{|c|}{2004} \\
\hline & & & & & & & & & & Q1 & Q2 \\
\hline & \multicolumn{11}{|c|}{ (In thousand of tons, except when otherwise noted) } \\
\hline Grains & 913 & 1,329 & 1,619 & 1,619 & 1,630 & 1,569 & 1,824 & 1,753 & 1,671 & $\ldots$ & 635 \\
\hline Wheat & 625 & 964 & 1,274 & 1,204 & 1,109 & 1,039 & 1,191 & 1,163 & 1,014 & $\ldots$ & 518 \\
\hline Barley & 159 & 166 & 152 & 162 & 180 & 150 & 140 & 149 & 198 & $\ldots$ & 113 \\
\hline Corn & 116 & 182 & 171 & 228 & 308 & 338 & 443 & 374 & 399 & $\ldots$ & $\ldots$ \\
\hline Rice & 7 & 9 & 12 & 11 & 15 & 19 & 17 & 21 & 18 & $\ldots$ & $\ldots$ \\
\hline Cotton & 75 & 73 & 62 & 78 & 87 & 88 & 98 & 106 & 106 & $\ldots$ & $\ldots$ \\
\hline Sugarbeet & 107 & 190 & 206 & 429 & 536 & 450 & 287 & 522 & 812 & $\ldots$ & $\ldots$ \\
\hline Tobacco & 18 & 18 & 26 & 28 & 30 & 35 & 24 & 6 & 9 & $\ldots$ & 0 \\
\hline Vegetable oil crop & 20 & 35 & 38 & 44 & 58 & 53 & 59 & 72 & 78 & $\ldots$ & 1 \\
\hline Potatoes & 432 & 562 & 678 & 774 & 957 & 1,046 & 1,168 & 1,244 & 1,308 & $\ldots$ & 82 \\
\hline Vegetables & 318 & 369 & 479 & 556 & 719 & 747 & 815 & 456 & 678 & $\ldots$ & 117 \\
\hline Melons & 23 & 41 & 38 & 47 & 63 & 65 & 84 & 42 & 85 & $\ldots$ & 19 \\
\hline Fruits and berries & 67 & 83 & 111 & 103 & 101 & 161 & 161 & 154 & 134 & $\ldots$ & 14 \\
\hline Grapes & 20 & 14 & 23 & 17 & 18 & 27 & 27 & 18 & 13 & $\ldots$ & 0 \\
\hline Meat (slaughtered) & 180 & 186 & 186 & 191 & 196 & 196 & 200 & 200 & 194 & 39 & 45 \\
\hline Milk & 864 & 885 & 912 & 973 & 1,064 & 1,105 & 1,142 & 1,173 & 1,192 & 193 & 344 \\
\hline Eggs (millions) & 147 & 160 & 164 & 176 & 193 & 207 & 228 & 243 & 268 & 55 & 96 \\
\hline \multirow[t]{2}{*}{ Wool } & 15 & 12 & 11 & 12 & 12 & 12 & 12 & 12 & 12 & - & 10 \\
\hline & \multicolumn{11}{|c|}{ (Percentage change from previous year) } \\
\hline Grains & -8.3 & 45.5 & 21.8 & 0.0 & 0.7 & -3.8 & 16.3 & -3.9 & -4.7 & $\ldots$ & 34.7 \\
\hline Wheat & 10.5 & 54.3 & 32.1 & -5.5 & -7.9 & -6.3 & 14.6 & -2.3 & -12.8 & $\ldots$ & 31.5 \\
\hline Barley & -44.9 & 4.7 & -8.9 & 6.7 & 11.2 & -16.5 & -6.8 & 6.7 & 32.6 & $\ldots$ & 48.8 \\
\hline Corn & -10.2 & 56.9 & -6.4 & 33.6 & 35.3 & 9.7 & 30.9 & -15.6 & 6.7 & $\ldots$ & $\ldots$ \\
\hline Rice & 71.8 & 37.3 & 27.2 & -6.0 & 36.4 & 26.7 & -10.5 & 25.3 & -12.0 & $\ldots$ & $\ldots$ \\
\hline Cotton & 39.3 & -1.9 & -14.6 & 24.7 & 11.7 & 1.2 & 11.5 & 8.4 & -0.5 & $\ldots$ & $\ldots$ \\
\hline Sugarbeet & -5.9 & 76.7 & 8.3 & 108.9 & 24.9 & -16.1 & -36.2 & 82.0 & 55.7 & $\ldots$ & $\ldots$ \\
\hline Tobacco & -51.6 & 1.7 & 43.6 & 9.3 & 6.0 & 16.1 & -30.6 & -74.6 & 42.6 & $\ldots$ & 257.9 \\
\hline Vegetable oil crop & 41.5 & 73.6 & 8.3 & 15.9 & 32.2 & -7.8 & 10.5 & 23.1 & 7.2 & $\ldots$ & 136.2 \\
\hline Potatoes & 38.8 & 30.3 & 20.6 & 14.1 & 23.7 & 9.2 & 11.7 & 6.5 & 5.2 & $\ldots$ & 26.2 \\
\hline Vegetables & 19.9 & 15.7 & 29.9 & 16.1 & 29.4 & 3.8 & 9.1 & -44.0 & 48.6 & $\ldots$ & 38.4 \\
\hline Melons & 23.3 & 73.8 & -5.4 & 21.7 & 34.8 & 4.0 & 28.6 & -49.5 & 102.1 & $\ldots$ & 63.5 \\
\hline Fruits and berries & -14.9 & 22.9 & 33.9 & -7.3 & -1.7 & 59.8 & -1.4 & -4.1 & -13.2 & $\ldots$ & 39.4 \\
\hline Grapes & 11.9 & -27.4 & 59.4 & -24.6 & 5.2 & 46.4 & 1.9 & -35.8 & -27.8 & $\ldots$ & 261.6 \\
\hline Meat (slaughtered) & -8.8 & 3.1 & 0.2 & 2.8 & 2.3 & 0.3 & 1.8 & 0.4 & -3.4 & -1.0 & 6.2 \\
\hline Milk & -0.8 & 2.4 & 3.0 & 6.7 & 9.4 & 3.8 & 3.3 & 2.7 & 1.6 & -0.9 & 0.2 \\
\hline Eggs & -27.2 & 8.8 & 2.7 & 7.3 & 9.8 & 7.5 & 9.8 & 6.7 & 10.1 & 9.9 & 7.1 \\
\hline Wool & -30.2 & -17.6 & -6.6 & 0.9 & 1.7 & 0.0 & 0.0 & -0.9 & 0.0 & 0.0 & -4.6 \\
\hline
\end{tabular}

Source: National Statistical Committee. 


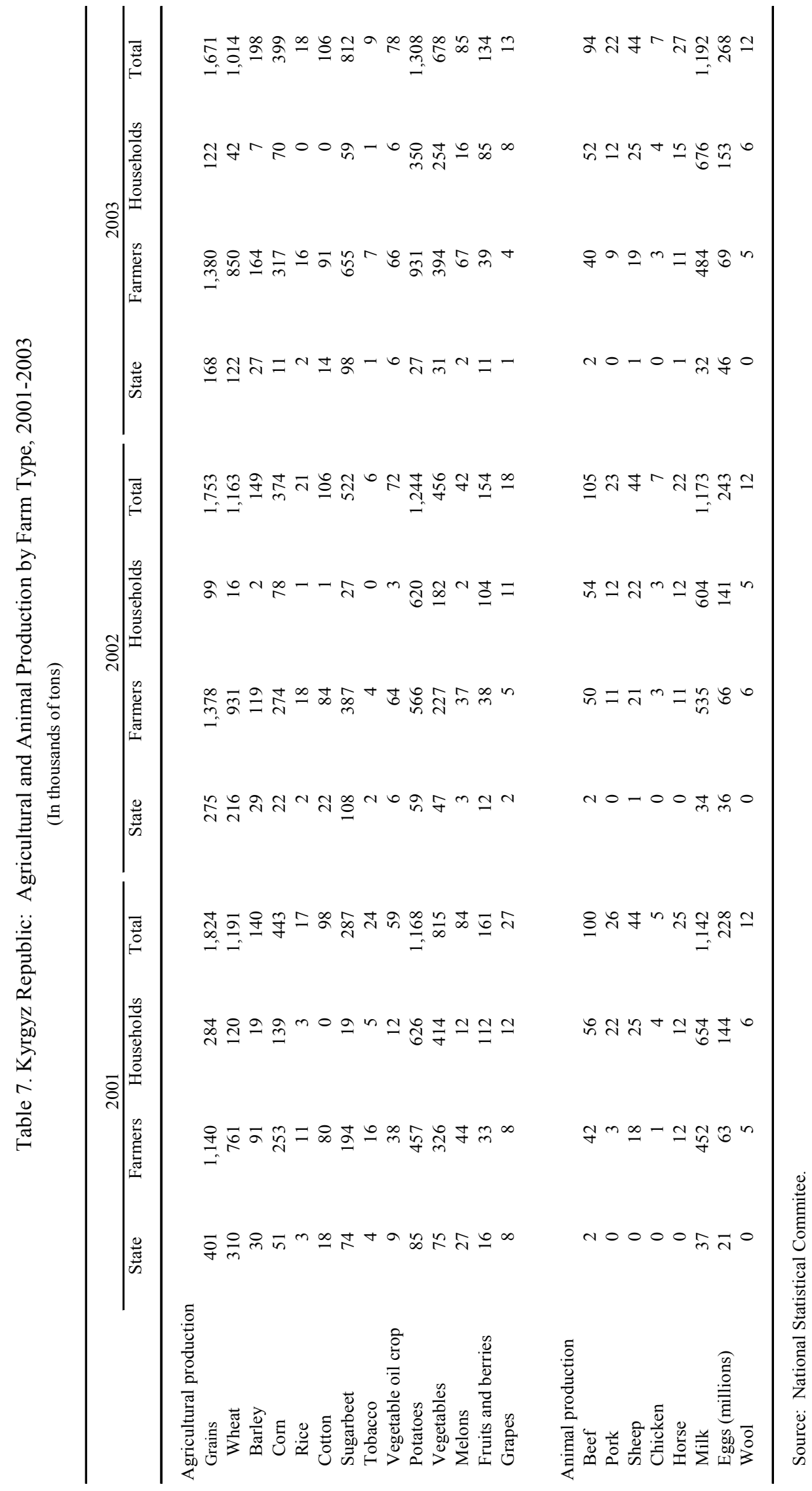


Table 8. Kyrgyz Republic: Yields of Major Agricultural Commodities, 1995-2003 (100 Kilogram per hectare)

\begin{tabular}{|c|c|c|c|c|c|c|c|c|c|}
\hline & 1995 & 1996 & 1997 & 1998 & 1999 & 2000 & 2001 & 2002 & 2003 \\
\hline Grains & 18 & 23 & 24 & 26 & 26 & 26 & 28 & 28 & 27 \\
\hline Wheat & 18 & 22 & 24 & 25 & 24 & 23 & 25 & 25 & 24 \\
\hline Barley & 13 & 17 & 19 & 22 & 20 & 21 & 21 & 24 & 23 \\
\hline Corn & 37 & 43 & 46 & 49 & 53 & 56 & 60 & 57 & 59 \\
\hline Rice & 16 & 18 & 19 & 22 & 25 & 26 & 27 & 27 & 28 \\
\hline Cotton & 22 & 23 & 25 & 25 & 25 & 26 & 26 & 27 & 26 \\
\hline Sugarbeet & 123 & 152 & 181 & 200 & 203 & 191 & 164 & 204 & 260 \\
\hline Tobacco & 21 & 21 & 21 & 22 & 25 & 24 & 25 & 25 & 24 \\
\hline Vegetable oil & 5 & 5 & 7 & 8 & 9 & 9 & 10 & 11 & 11 \\
\hline Potatoes & 99 & 114 & 121 & 131 & 150 & 151 & 157 & 158 & 155 \\
\hline Vegetables & 103 & 113 & 132 & 143 & 152 & 157 & 165 & 175 & 178 \\
\hline Melons & 65 & 83 & 121 & 123 & 150 & 164 & 162 & 183 & 192 \\
\hline Fruits and berries & 21 & 24 & 27 & 25 & 24 & 38 & 37 & 36 & 32 \\
\hline Grapes & 30 & 22 & 31 & 23 & 24 & 37 & 37 & 24 & 20 \\
\hline
\end{tabular}

Source: National Statistical Committee. 
Table 9. Kyrgyz Republic: Consumer and Producer Prices, 1998-2004 1/

\begin{tabular}{|c|c|c|c|c|}
\hline & \multicolumn{2}{|c|}{ Consumer Price Index } & \multicolumn{2}{|c|}{ Producer Price Index } \\
\hline & $\begin{array}{l}\text { Percent } \\
\text { change }\end{array}$ & $\begin{array}{c}\text { Index } \\
(1994=100)\end{array}$ & $\begin{array}{l}\text { Percent } \\
\text { change }\end{array}$ & $\begin{array}{c}\text { Index } \\
(1994=100)\end{array}$ \\
\hline 1998 Average 1/ & 10.5 & 258.1 & 9.0 & 200.0 \\
\hline 1999 Average 1/ & 35.9 & 350.8 & 51.3 & 302.6 \\
\hline 2000 Average 1/ & 18.7 & 416.4 & 29.7 & 392.4 \\
\hline 2001 January & 0.9 & 435.4 & -1.0 & 413.5 \\
\hline February & 1.3 & 441.1 & 0.6 & 416.0 \\
\hline March & 0.6 & 443.6 & 3.1 & 429.1 \\
\hline April & 1.7 & 450.9 & -0.2 & 428.1 \\
\hline May & 2.3 & 461.2 & 1.2 & 433.1 \\
\hline June & -0.9 & 457.1 & 0.4 & 435.0 \\
\hline July & -1.8 & 448.6 & 0.6 & 437.5 \\
\hline August & -2.4 & 437.9 & -0.6 & 434.9 \\
\hline September & 0.0 & 438.0 & 0.2 & 435.8 \\
\hline October & 0.4 & 439.7 & -0.5 & 433.7 \\
\hline November & 0.3 & 441.1 & -0.4 & 431.8 \\
\hline December & 1.4 & 447.4 & 0.2 & 432.6 \\
\hline 2001 Average 1/ & 6.9 & 445.2 & 9.6 & 430.1 \\
\hline 2002 January & 0.6 & 450.0 & 0.9 & 436.4 \\
\hline February & 0.3 & 451.3 & 0.1 & 436.8 \\
\hline March & -0.1 & 451.1 & 1.8 & 444.8 \\
\hline April & 0.7 & 454.2 & 1.0 & 449.1 \\
\hline May & 1.1 & 459.4 & 1.3 & 454.8 \\
\hline June & 0.4 & 461.4 & 1.8 & 463.2 \\
\hline July & -1.2 & 456.0 & 0.0 & 463.2 \\
\hline August & -0.8 & 452.6 & -2.3 & 452.6 \\
\hline September & 0.1 & 453.1 & 1.5 & 459.6 \\
\hline October & -0.3 & 451.9 & 0.0 & 459.5 \\
\hline November & 0.3 & 453.2 & 0.2 & 460.4 \\
\hline December & 1.0 & 457.7 & 0.7 & 463.6 \\
\hline 2002 Average 1/ & 2.1 & 454.3 & 5.5 & 453.7 \\
\hline 2003 January & 0.9 & 461.8 & 3.3 & 478.9 \\
\hline February & 0.4 & 463.9 & 2.3 & 489.7 \\
\hline March & 0.7 & 466.9 & -2.0 & 479.8 \\
\hline April & 1.0 & 471.6 & -2.2 & 469.2 \\
\hline May & 1.4 & 478.2 & 0.3 & 470.7 \\
\hline June & -1.9 & 469.2 & -0.3 & 469.1 \\
\hline July & -1.7 & 461.0 & 1.4 & 475.8 \\
\hline August & -0.7 & 457.9 & 1.9 & 484.9 \\
\hline September & -0.1 & 457.4 & 1.5 & 492.0 \\
\hline October & 2.6 & 469.4 & 3.1 & 507.1 \\
\hline November & 2.1 & 479.2 & -0.1 & 506.8 \\
\hline December & 0.8 & 483.2 & 3.8 & 526.3 \\
\hline 2003 Average 1/ & 3.1 & 468.3 & 7.5 & 487.5 \\
\hline 2004 January & 1.0 & 488.1 & 0.9 & 530.9 \\
\hline February & 0.2 & 489.1 & -2.6 & 517.2 \\
\hline March & 0.1 & 489.6 & 1.4 & 524.4 \\
\hline April & -0.7 & 486.4 & 2.1 & 535.2 \\
\hline May & 0.0 & 486.6 & -1.3 & 528.0 \\
\hline June & 0.3 & 488.0 & -1.1 & 522.1 \\
\hline July & -0.2 & 487.0 & 1.2 & 528.2 \\
\hline August & -0.9 & 482.6 & -0.3 & 526.8 \\
\hline
\end{tabular}

Source: National Statistical Committee.

$1 /$ Changes refer to annual average data. 


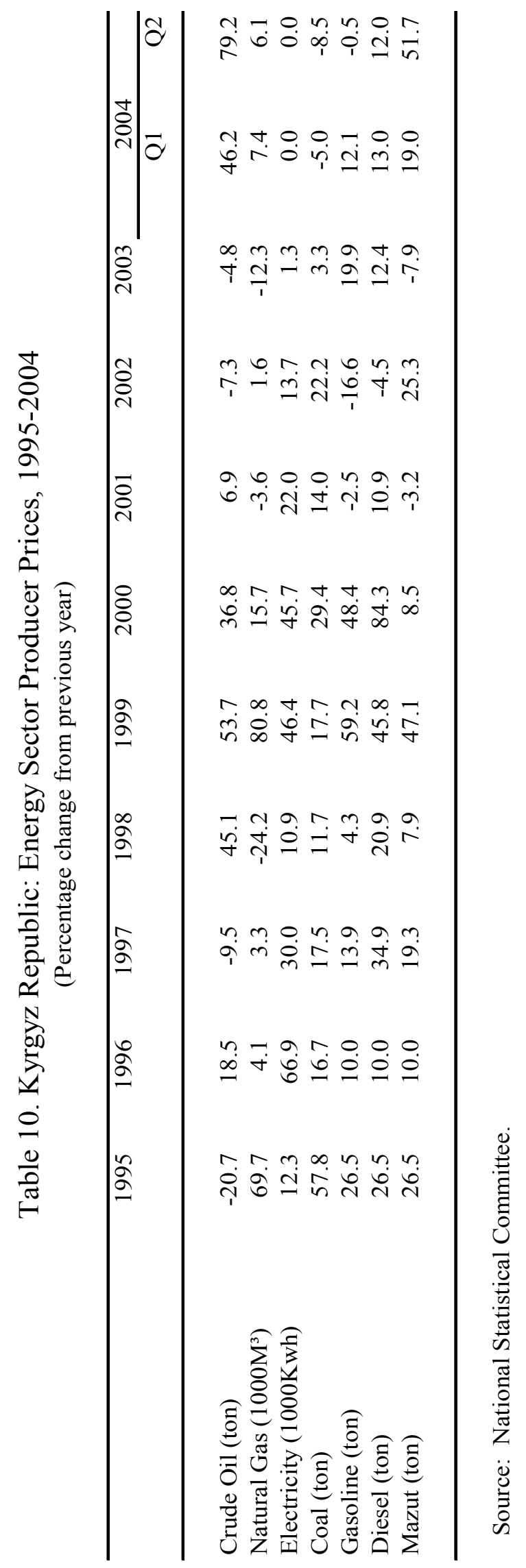


Table 11. Kyrgyz Republic: Nominal and Real Wages, 1995-2004

\begin{tabular}{|c|c|c|c|c|}
\hline & \multicolumn{2}{|c|}{$\begin{array}{l}\text { Nominal wages } \\
\text { (In soms) } 1 /\end{array}$} & \multicolumn{2}{|c|}{$\begin{array}{c}\text { Index of real wages } \\
(1994=100)\end{array}$} \\
\hline & Average & Minimum & Average & Minimum \\
\hline 1995 Average 2/ & 390.3 & 69.2 & 117.4 & 82.9 \\
\hline Average 3/ & 368.2 & $\ldots$ & 110.0 & .. \\
\hline 1996 Average 2/ & 493.7 & 75.0 & 112.8 & 68.1 \\
\hline Average 3/ & 490.9 & $\ldots$ & 111.1 & $\cdots$ \\
\hline 1997 Average 2/ & 630.4 & 82.5 & 116.4 & 60.7 \\
\hline Average 3/ & 680.2 & $\ldots$ & 124.7 & $\ldots$ \\
\hline 1998 Average 2/ & 785.2 & 100.0 & 131.0 & 66.6 \\
\hline Average 3/ & 840.6 & 100.0 & 139.6 & 66.6 \\
\hline 1999 Average 2/ & 976.3 & 100.0 & 120.1 & 49.0 \\
\hline Average 3/ & $1,049.9$ & 100.0 & 128.2 & 49.0 \\
\hline 2000 Average 2/ & $1,176.6$ & 100.0 & 123.3 & 41.3 \\
\hline Average 3/ & $1,227.0$ & 100.0 & 126.3 & 41.3 \\
\hline 2001 Average 2/ & $1,392.7$ & 100.0 & 134.3 & 33.0 \\
\hline Average 3/ & $1,455.1$ & 100.0 & 140.0 & 33.0 \\
\hline 20021 Quarter & $1,423.3$ & 100.0 & 135.3 & 32.6 \\
\hline 2 Quarter & $1,574.5$ & 100.0 & 147.2 & 32.1 \\
\hline 3 Quarter & $1,623.1$ & 100.0 & 153.2 & 32.4 \\
\hline 4 Quarter & $1,841.7$ & 100.0 & 173.7 & 32.4 \\
\hline Average 2/ & $1,617.6$ & 100.0 & 152.6 & 32.4 \\
\hline Average 3/ & $1,684.4$ & 100.0 & 158.9 & 32.4 \\
\hline 20031 Quarter & $1,683.1$ & 100.0 & 155.3 & 31.7 \\
\hline 2 Quarter & $1,886.9$ & 100.0 & 170.9 & 31.1 \\
\hline 3 Quarter & $1,952.2$ & 100.0 & 182.3 & 32.0 \\
\hline 4 Quarter & $2,103.6$ & 100.0 & 188.8 & 30.8 \\
\hline Average 2/ & $1,901.5$ & 100.0 & 174.0 & 31.4 \\
\hline Average 3/ & $1,916.0$ & 100.0 & 175.3 & 31.4 \\
\hline 2004 January & $1,871.6$ & 100.0 & 164.3 & 30.1 \\
\hline February & $1,947.5$ & 100.0 & 170.6 & 30.1 \\
\hline March & $2,067.1$ & 100.0 & 180.9 & 30.0 \\
\hline April & $2,049.7$ & 100.0 & 180.5 & 30.2 \\
\hline May & $2,071.8$ & 100.0 & 182.4 & 30.2 \\
\hline June & $2,306.6$ & 100.0 & 202.5 & 30.1 \\
\hline July & $2,248.7$ & 100.0 & 197.8 & 30.2 \\
\hline
\end{tabular}

Source: National Statistical Committee.

1/ The December average wage reflects year-end bonus, typically one month's wage.

2/ Based on the monthly wage statistics.

3/ Based on annual wage statistics with a broader coverage of the sectors than the monthly statistics, particularly with respect to agriculture. 


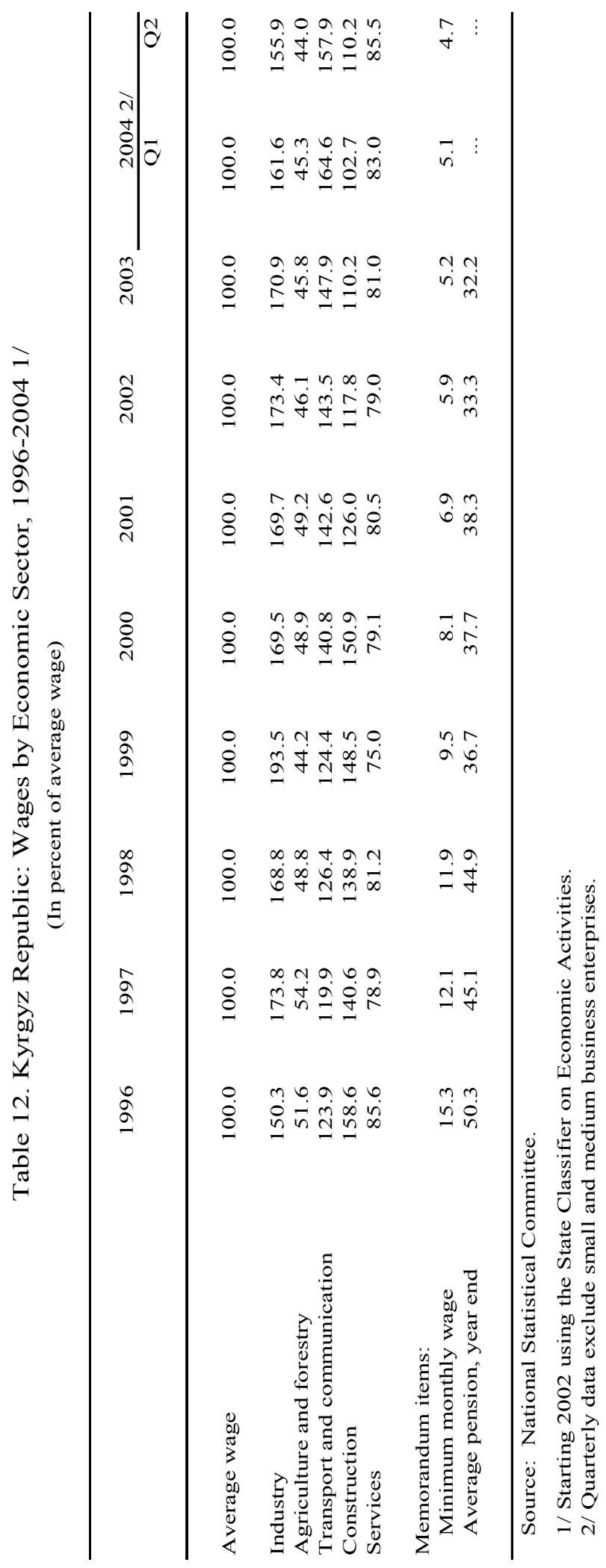




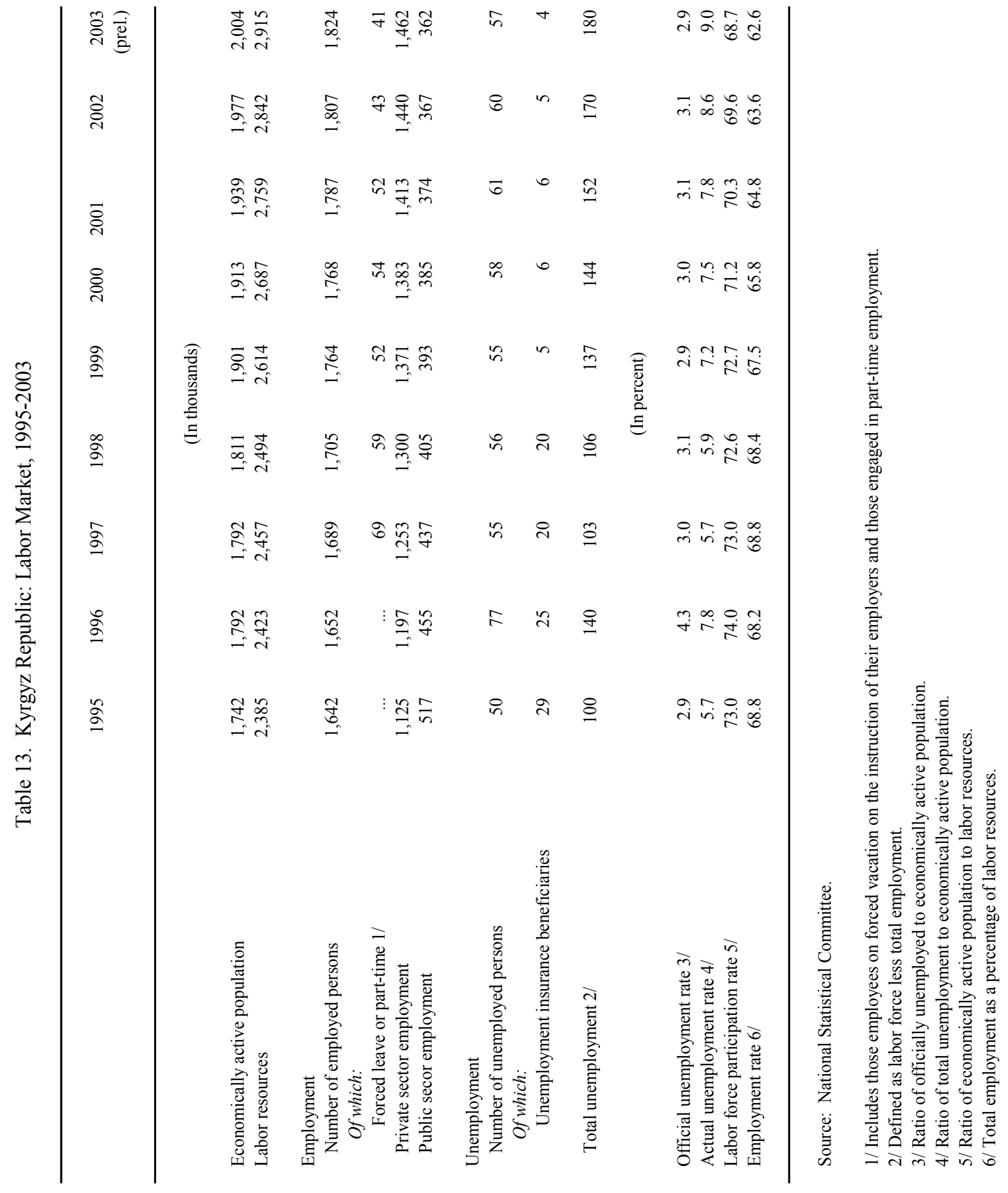




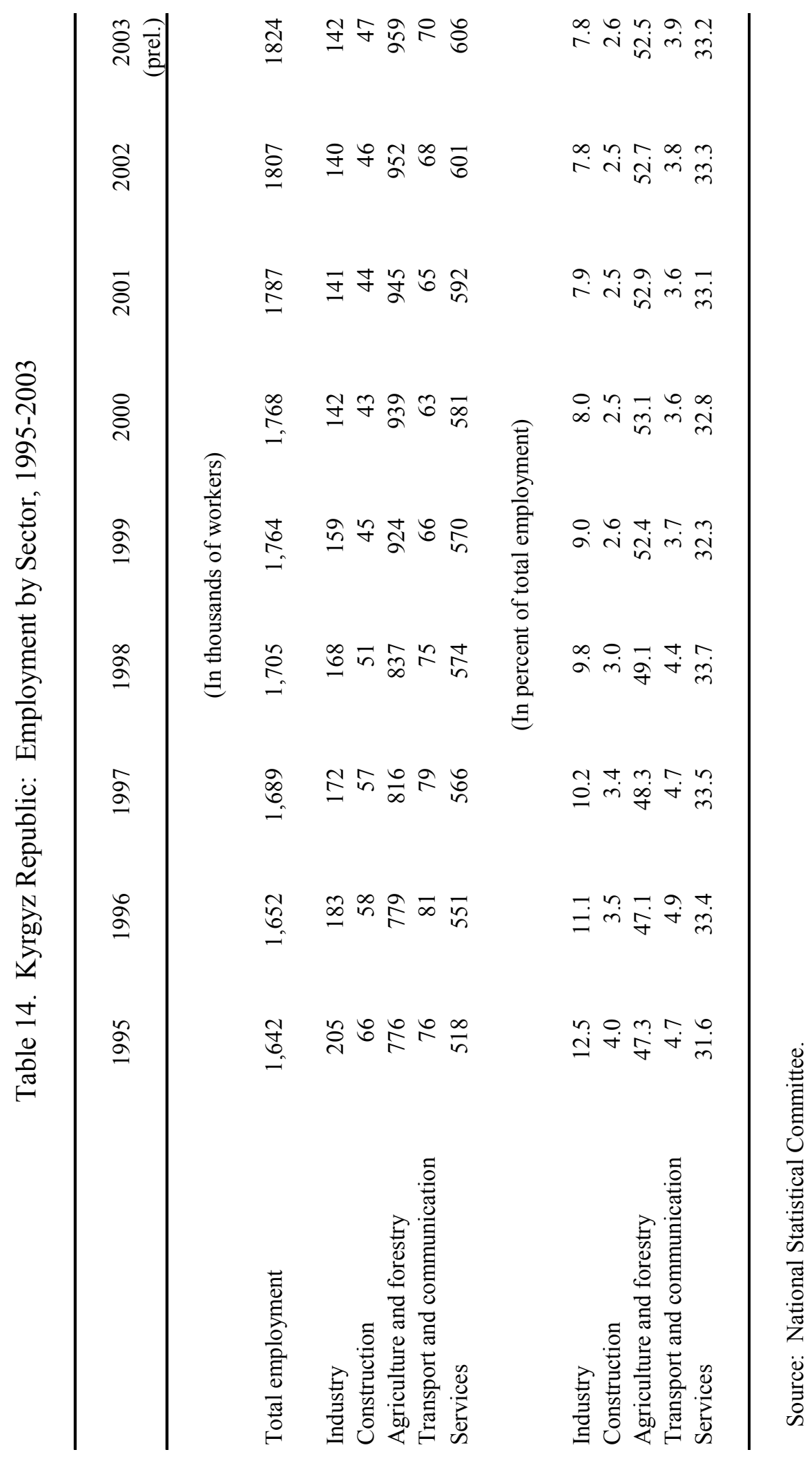




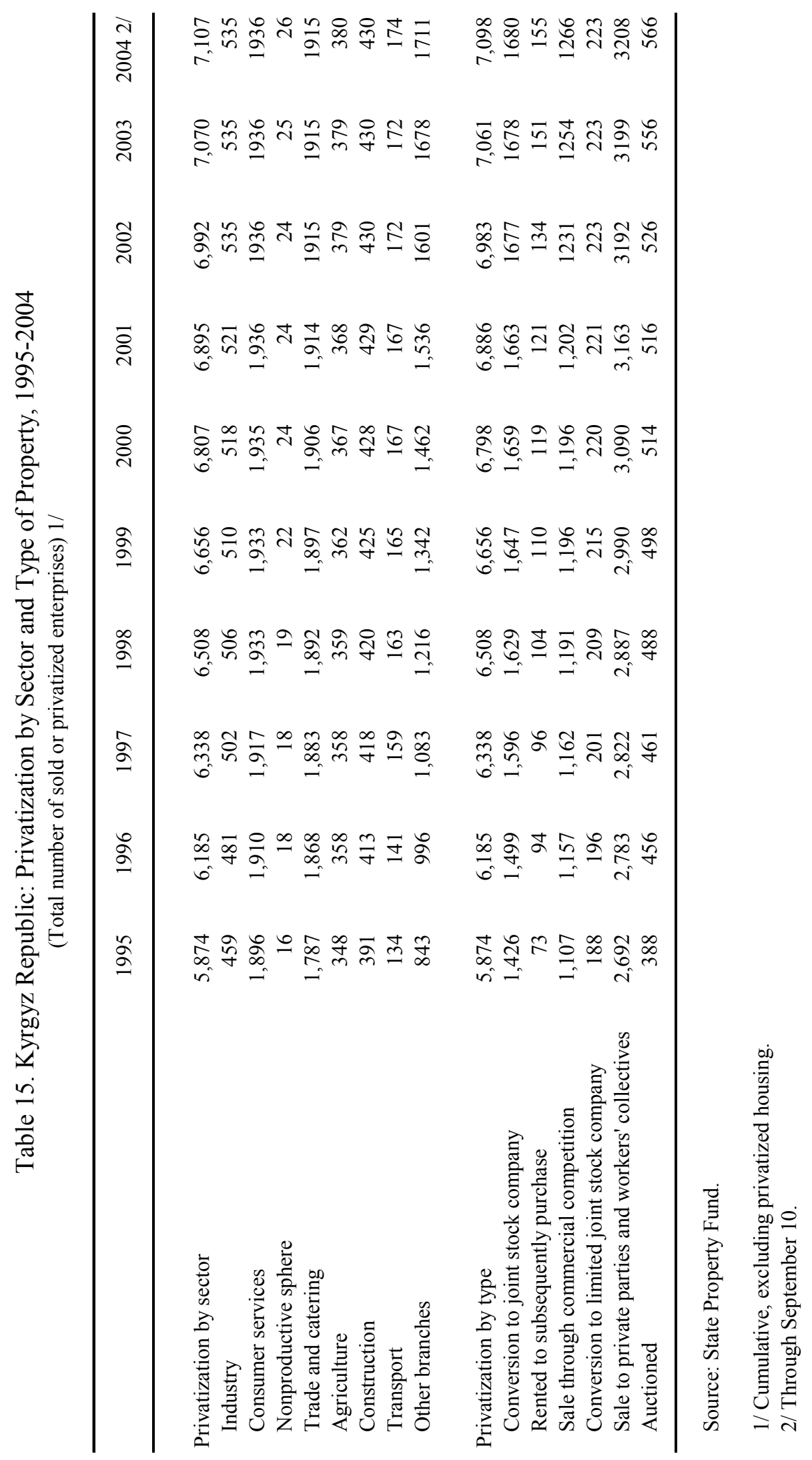


Table 16. Kyrgyz Republic: Balance of Payments 1995-2004 (Q2) (In millions of U.S. dollars)

\begin{tabular}{|c|c|c|c|c|c|c|c|c|c|c|c|c|c|c|c|c|}
\hline & \multirow[t]{2}{*}{1995} & \multirow[t]{2}{*}{1996} & \multirow[t]{2}{*}{1997} & \multirow[t]{2}{*}{1998} & \multirow[t]{2}{*}{1999} & \multirow[t]{2}{*}{2000} & \multirow[t]{2}{*}{2001} & \multirow[t]{2}{*}{2002} & \multirow[t]{2}{*}{2003} & \multicolumn{4}{|c|}{2003} & \multirow[t]{2}{*}{2004} & \multicolumn{2}{|c|}{2004} \\
\hline & & & & & & & & & & Q1 & Q2 & Q3 & $\overline{\mathrm{Q} 4}$ & & Q1 & $\mathrm{Q} 2^{2}$ \\
\hline Current account balance 1/ & -263.8 & -440.7 & -146.7 & -372.0 & -199.1 & -91.0 & -51.0 & -37.6 & -26.7 & 16.2 & -42.3 & -9.0 & 8.5 & -14.6 & -8.5 & -6.1 \\
\hline Trade balance $2 /$ & -122.0 & -251.7 & -15.2 & -220.7 & -88.6 & 4.0 & 39.9 & -54.0 & -82.7 & 3.4 & -41.0 & -39.6 & -5.5 & -51.0 & -24.7 & -26.3 \\
\hline Exports, fob & 408.9 & 531.2 & 630.8 & 535.1 & 462.6 & 510.9 & 480.3 & 498.1 & 590.3 & 121.3 & 115.4 & 142.1 & 211.6 & 342.6 & 159.0 & 183.7 \\
\hline CIS countries & 269.2 & 393.9 & 346.3 & 252.0 & 191.5 & 213.7 & 172.2 & 170.3 & 202.4 & 39.8 & 38.7 & 48.6 & 75.3 & 116.6 & 54.8 & 61.8 \\
\hline Energy & 45.6 & 77.9 & 86.5 & 28.3 & 53.1 & 82.0 & 51.5 & 28.0 & 25.9 & 3.0 & 2.7 & 11.8 & 8.4 & 8.5 & 2.4 & 6.2 \\
\hline Other & 223.6 & 315.9 & 259.8 & 223.8 & 138.4 & 131.7 & 120.7 & 142.3 & 176.4 & 36.9 & 36.0 & 36.8 & 66.8 & 108.1 & 52.4 & 55.7 \\
\hline Of which: Gold & 0.0 & 0.0 & 184.4 & 195.8 & 183.1 & 195.3 & 224.6 & 162.8 & 259.6 & 53.6 & 46.4 & 68.1 & 91.6 & 150.0 & 67.3 & 82.7 \\
\hline Imports, fob & 530.9 & 782.9 & 646.0 & 755.7 & 551.1 & 506.9 & 440.4 & 552.1 & 673.0 & 117.9 & 156.3 & 181.7 & 217.1 & 393.6 & 183.6 & 210.0 \\
\hline CIS countries & 313.1 & 431.1 & 396.0 & 391.7 & 248.7 & 279.7 & 250.0 & 311.7 & 394.5 & 64.3 & 91.8 & 110.6 & 127.7 & 244.3 & 113.7 & 130.7 \\
\hline Energy & 163.1 & 207.7 & 177.4 & 173.8 & 113.2 & 120.4 & 116.7 & 145.3 & 172.4 & 30.1 & 37.9 & 43.7 & 60.6 & 107.3 & 56.5 & 50.8 \\
\hline Other & 150.0 & 223.4 & 218.5 & 217.8 & 135.6 & 159.3 & 133.4 & 166.4 & 222.1 & 34.2 & 53.9 & 66.9 & 67.2 & 137.0 & 57.2 & 79.8 \\
\hline Other countries & 217.8 & 351.7 & 250.1 & 364.1 & 302.4 & 227.2 & 190.3 & 240.3 & 278.6 & 53.5 & 64.5 & 71.1 & 89.3 & 149.3 & 70.0 & 79.3 \\
\hline Of which: Gold (Kumtor) & 44.4 & 77.1 & 25.0 & 24.2 & 45.2 & 10.7 & 0.0 & & & & & & & & & \\
\hline Services (net) & -191.4 & -257.0 & -190.8 & -192.0 & -163.4 & -171.0 & -110.0 & -64.1 & -54.8 & -10.3 & -18.8 & 3.3 & -29.1 & -42.9 & -26.1 & -16.8 \\
\hline Non-interest service & -156.0 & -217.5 & -126.1 & -112.8 & -89.4 & -86.9 & -44.4 & -5.4 & 7.0 & -3.7 & -2.2 & 9.3 & 3.7 & -4.7 & 0.8 & -5.5 \\
\hline Receipts & 39.1 & 31.5 & 45.0 & 62.8 & 64.9 & 61.8 & 80.3 & 142.0 & 154.9 & 27.3 & 34.3 & 46.7 & 46.7 & 80.3 & 43.2 & 37.1 \\
\hline Of which: Technical assistance & -48.3 & -44.8 & -23.3 & -19.8 & -18.5 & -20.4 & -21.3 & -21.0 & -20.2 & -5.1 & -5.0 & -5.1 & -5.0 & -10.4 & -5.1 & -5.2 \\
\hline Interest payments (scheduled) & -29.7 & -34.3 & -56.7 & -65.7 & -67.3 & -51.2 & -39.4 & -32.0 & -27.1 & -2.4 & -10.5 & -2.7 & -11.5 & -20.2 & -8.2 & -11.9 \\
\hline Other net income & -5.7 & -5.1 & -8.0 & -13.4 & -6.7 & -32.8 & -26.2 & -26.7 & -34.8 & -4.2 & -6.0 & -3.3 & -21.3 & -18.1 & -18.7 & 0.7 \\
\hline Transfers (net) & 49.6 & 68.0 & 59.3 & 40.6 & 52.8 & 76.0 & 19.1 & 80.4 & 110.8 & 23.0 & 17.4 & 27.3 & 43.0 & 79.2 & 42.3 & 37.0 \\
\hline Official $3 /$ & 48.8 & 66.2 & 57.1 & 51.0 & 73.9 & 55.6 & -0.4 & 21.9 & 16.2 & 7.6 & 1.5 & 4.3 & 2.8 & 15.7 & 11.8 & 3.9 \\
\hline Private & 0.8 & 1.8 & 2.2 & -10.4 & -21.1 & 20.4 & 19.6 & 58.5 & 94.6 & 15.5 & 15.9 & 23.1 & 40.2 & 63.6 & 30.5 & 33.1 \\
\hline Capital account balance & 260.0 & 362.3 & 249.9 & 284.4 & 220.6 & 65.3 & 32.9 & 26.5 & -25.5 & 3.2 & 1.4 & -10.5 & -19.6 & 73.3 & -7.1 & 80.4 \\
\hline Commercial banks & -4.5 & -0.5 & -4.7 & 10.6 & -4.2 & -1.9 & -12.5 & -9.6 & -13.5 & -5.3 & -2.5 & -13.9 & 8.1 & -12.8 & -9.9 & -2.9 \\
\hline Medium-and long-term loans, net & 192.1 & 308.5 & 148.1 & 105.6 & 133.3 & 41.7 & 17.0 & 34.4 & -22.9 & 9.0 & 1.1 & 23.3 & -56.2 & -13.5 & -15.8 & 2.2 \\
\hline Disbursement & 264.4 & 359.0 & 163.2 & 158.6 & 196.6 & 131.3 & 118.2 & 90.7 & 80.4 & 15.8 & 14.5 & 28.7 & 21.4 & 27.9 & 8.4 & 19.5 \\
\hline Amortization (scheduled) & -72.4 & -50.5 & -15.1 & -52.9 & -63.3 & -89.5 & -101.2 & -56.3 & -103.3 & -6.8 & -13.4 & -5.5 & -77.6 & -41.4 & -24.1 & -17.3 \\
\hline Foreign direct investment & 96.1 & 46.8 & 83.0 & 86.6 & 38.4 & -6.9 & -1.1 & 4.7 & 45.5 & 4.2 & 1.5 & 5.9 & 33.9 & 101.7 & 15.8 & 85.9 \\
\hline Other assets 4 / & -23.7 & 7.5 & 23.5 & 81.6 & 53.1 & 32.3 & 29.5 & 57.3 & -34.6 & -4.7 & 1.2 & -25.9 & -5.3 & -2.0 & 2.8 & -4.8 \\
\hline Errors and omissions and short-term capital & -78.3 & 57.9 & -57.6 & 63.6 & -3.4 & 9.6 & -24.2 & 32.4 & 54.3 & -36.4 & 32.2 & 26.9 & 31.5 & 3.6 & 16.2 & -12.6 \\
\hline Overall balance & -82.1 & -20.5 & 45.6 & -24.0 & 18.1 & -16.2 & -42.3 & 21.3 & 2.2 & -16.9 & -8.7 & 7.4 & 20.4 & 62.3 & 0.6 & 61.6 \\
\hline Financing & 82.1 & 20.5 & -45.6 & 24.0 & -18.1 & 16.2 & 42.3 & -21.3 & -2.2 & 16.9 & 8.7 & -7.4 & -20.4 & -62.3 & -0.6 & -61.6 \\
\hline Purchases and disbursements & 46.6 & 23.5 & 43.9 & 14.2 & 26.8 & 18.7 & 14.9 & 15.4 & 29.5 & 16.1 & 0.0 & 13.4 & 0.0 & 14.0 & 14.0 & 0.0 \\
\hline Repurchases and repayments & 0.0 & -3.9 & -9.5 & -11.6 & -7.3 & -11.4 & -17.0 & -21.4 & -30.4 & -9.0 & -8.7 & -3.6 & -9.1 & -14.6 & -5.4 & -9.2 \\
\hline Exceptional financing (including arrears) & 34.0 & 18.8 & 2.6 & 15.5 & 23.7 & 30.1 & 19.7 & 20.9 & 44.4 & 6.0 & 14.8 & 5.7 & 17.9 & 24.6 & 7.6 & 17.0 \\
\hline BOP support loans (ADB) 6/ & 0.0 & 0.0 & 0.0 & 0.0 & 0.0 & 0.0 & 33.4 & 7.6 & 0.0 & 0.0 & 0.0 & 0.0 & 0.0 & 0.0 & 0.0 & 0.0 \\
\hline BoP support loans (WB) 6/ & 0.0 & 0.0 & 0.0 & 0.0 & 0.0 & 0.0 & 7.5 & 0.0 & 5.2 & 0.0 & 0.0 & 5.2 & 0.0 & 0.0 & 0.0 & 0.0 \\
\hline
\end{tabular}

Sources: Kyrgyz authorities.

$1 /$ Including transfers.

2/ Excluding any import adjustment made to account for unrecorded imports.

3 / Technical assistance is shown in services rather than transfers.

4/ Including accounts payable and receivable.

5/ Valued at end-year exchange rates. Gross reserves exclude NBKR pledges to secure government-guaranteed loans and blocked deposits.

6/ BOP support loans before 2001 are shown as disbursements under the capital account. 
Table 17. Kyrgyz Republic: Exports of Goods by Product Category, 1995-2004

\begin{tabular}{|c|c|c|c|c|c|c|c|c|c|c|}
\hline & 1995 & 1996 & 1997 & 1998 & 1999 & 2000 & 2001 & 2002 & 2003 & $\begin{array}{r}2004 \\
\text { first half } \\
\end{array}$ \\
\hline & \multicolumn{10}{|c|}{ (In millions of U.S. dollars) } \\
\hline Total exports $1 /$ & 408.9 & 505.4 & 603.8 & 513.6 & 453.8 & 504.5 & 476.2 & 485.5 & 581.7 & 338.3 \\
\hline Industry & 366.1 & 442.1 & 558.6 & 461.1 & 396.9 & 457.5 & 430.8 & 429.3 & 551.1 & 321.5 \\
\hline Electric energy & 41.0 & 73.6 & 83.2 & 25.6 & 52.0 & 79.8 & 46.8 & 22.0 & 19.2 & 6.2 \\
\hline Oil and gas industry & 1.5 & 2.2 & 2.3 & 2.9 & 1.4 & 2.5 & 8.0 & 35.4 & 47.9 & 23.4 \\
\hline Coal industry & 3.1 & 2.0 & 1.8 & 0.5 & 0.2 & 0.3 & 0.3 & 0.8 & 0.2 & 0.2 \\
\hline Ferrous metallurgy & 10.4 & 6.9 & 3.0 & 2.9 & 1.6 & 5.4 & 6.0 & 5.2 & 6.4 & 9.6 \\
\hline Nonferrous metallurgy & 62.7 & 56.7 & 216.2 & 221.0 & 217.2 & 233.9 & 246.0 & 197.2 & 278.7 & 168.0 \\
\hline Chemical and petrochemical & 20.3 & 13.8 & 14.8 & 8.6 & 6.9 & 12.4 & 6.8 & 14.0 & 15.8 & 9.0 \\
\hline Machine building & 44.5 & 56.2 & 61.6 & 70.1 & 46.8 & 50.7 & 57.3 & 53.6 & 45.1 & 23.0 \\
\hline Lumber and paper & 1.8 & 2.4 & 3.6 & 4.6 & 0.7 & 1.0 & 0.6 & 1.3 & 2.3 & 1.7 \\
\hline Industrial construction materials & 11.6 & 21.8 & 26.9 & 24.0 & 8.2 & 8.4 & 8.4 & 12.7 & 33.3 & 20.8 \\
\hline Light industry & 82.5 & 74.4 & 60.7 & 40.8 & 32.3 & 43.9 & 32.1 & 64.9 & 73.9 & 34.8 \\
\hline Food industry & 79.1 & 127.0 & 79.6 & 54.5 & 19.1 & 13.6 & 12.5 & 17.0 & 24.6 & 22.2 \\
\hline Other industry & 7.6 & 5.2 & 5.0 & 5.6 & 10.4 & 5.7 & 6.1 & 5.1 & 3.6 & 2.6 \\
\hline Agriculture & 42.9 & 63.2 & 45.2 & 52.5 & 56.8 & 46.9 & 45.3 & 56.1 & 30.4 & 16.8 \\
\hline \multirow[t]{2}{*}{ Other } & 0.0 & 0.1 & 0.0 & 0.0 & 0.2 & 0.1 & 0.1 & 0.2 & 0.1 & 0.1 \\
\hline & \multicolumn{10}{|c|}{ (In percent of total) } \\
\hline Total exports & 100.0 & 100.0 & 100.0 & 100.0 & 100.0 & 100.0 & 100.0 & 100.0 & 100.0 & 100.0 \\
\hline Industry & 89.5 & 87.5 & 92.5 & 89.8 & 87.4 & 90.7 & 90.5 & 88.4 & 94.7 & 95.0 \\
\hline Electric energy & 10.0 & 14.6 & 13.8 & 5.0 & 11.5 & 15.8 & 9.8 & 4.5 & 3.3 & 1.8 \\
\hline Oil and gas industry & 0.4 & 0.4 & 0.4 & 0.6 & 0.3 & 0.5 & 1.7 & 7.3 & 8.2 & 6.9 \\
\hline Coal industry & 0.8 & 0.4 & 0.3 & 0.1 & 0.0 & 0.1 & 0.1 & 0.2 & 0.0 & 0.1 \\
\hline Ferrous metallurgy & 2.5 & 1.4 & 0.5 & 0.6 & 0.3 & 1.1 & 1.3 & 1.1 & 1.1 & 2.8 \\
\hline Nonferrous metallurgy & 15.3 & 11.2 & 35.8 & 43.0 & 47.9 & 46.4 & 51.7 & 40.6 & 47.9 & 49.6 \\
\hline Chemical and petrochemical & 5.0 & 2.7 & 2.4 & 1.7 & 1.5 & 2.5 & 1.4 & 2.9 & 2.7 & 2.7 \\
\hline Machine building & 10.9 & 11.1 & 10.2 & 13.6 & 10.3 & 10.1 & 12.0 & 11.0 & 7.8 & 6.8 \\
\hline Lumber and paper & 0.4 & 0.5 & 0.6 & 0.9 & 0.2 & 0.2 & 0.1 & 0.3 & 0.4 & 0.5 \\
\hline Industrial construction materials & 2.8 & 4.3 & 4.4 & 4.7 & 1.8 & 1.7 & 1.8 & 2.6 & 5.7 & 6.1 \\
\hline Light industry & 20.2 & 14.7 & 10.0 & 7.9 & 7.1 & 8.7 & 6.7 & 13.4 & 12.7 & 10.3 \\
\hline Food industry & 19.3 & 25.1 & 13.2 & 10.6 & 4.2 & 2.7 & 2.6 & 3.5 & 4.2 & 6.6 \\
\hline Other industry & 1.9 & 1.0 & 0.8 & 1.1 & 2.3 & 1.1 & 1.3 & 1.1 & 0.6 & 0.8 \\
\hline Agriculture & 10.5 & 12.5 & 7.5 & 10.2 & 12.5 & 9.3 & 9.5 & 11.6 & 5.2 & 5.0 \\
\hline Other & 0.0 & 0.0 & 0.0 & 0.0 & 0.0 & 0.0 & 0.0 & 0.0 & 0.0 & 0.0 \\
\hline
\end{tabular}

Sources: Kyrgyz authorities; and Fund staff estimates.

1/ Data for 1995 and 1996 do not include any estimates of unrecorded exports to other CIS countries due to incomplete coverage. 
Table 18. Kyrgyz Republic: Imports of Goods by Product Category, 1995-2004

\begin{tabular}{|c|c|c|c|c|c|c|c|c|c|c|}
\hline & 1995 & 1996 & 1997 & 1998 & 1999 & 2000 & 2001 & 2002 & 2003 & $\begin{array}{r}2004 \\
\text { first half } \\
\end{array}$ \\
\hline & \multicolumn{10}{|c|}{ (In millions of U.S. dollars) } \\
\hline Total imports (c.i.f.) $1 /$ & 522.3 & 837.7 & 709.3 & 841.5 & 599.7 & 554.6 & 467.2 & 586.8 & 717.0 & 421.8 \\
\hline Industry & 505.4 & 809.9 & 674.7 & 822.9 & 568.3 & 513.7 & 453.1 & 569.4 & 704.0 & 414.5 \\
\hline Electric energy & 8.6 & 26.7 & 23.8 & 7.9 & 2.8 & 7.6 & 9.8 & 9.7 & 0.5 & 0.0 \\
\hline Oil and gas industry & 162.6 & 187.6 & 175.9 & 181.5 & 99.1 & 110.8 & 105.5 & 129.5 & 159.0 & 109.3 \\
\hline Coal industry & 17.0 & 25.1 & 7.0 & 17.5 & 19.6 & 10.8 & 6.2 & 13.6 & 21.4 & 4.6 \\
\hline Ferrous metallurgy & 17.8 & 15.0 & 9.9 & 21.6 & 9.5 & 11.0 & 9.6 & 14.5 & 19.4 & 11.0 \\
\hline Nonferrous metallurgy & 11.2 & 8.7 & 22.4 & 30.6 & 22.9 & 12.5 & 24.0 & 27.7 & 17.5 & 16.1 \\
\hline Chemical and petrochemical & 30.1 & 51.0 & 59.8 & 75.5 & 38.8 & 48.1 & 44.4 & 73.3 & 73.6 & 43.0 \\
\hline Machine building & 103.6 & 230.5 & 154.0 & 219.3 & 202.4 & 144.9 & 98.2 & 106.5 & 144.3 & 84.6 \\
\hline Lumber and paper & 19.8 & 26.2 & 29.2 & 35.3 & 19.1 & 26.8 & 20.9 & 22.3 & 34.7 & 19.8 \\
\hline Industrial construction materials & 10.1 & 15.8 & 13.3 & 16.0 & 11.0 & 8.7 & 10.0 & 20.5 & 19.5 & 11.3 \\
\hline Light industry & 23.2 & 16.6 & 48.4 & 60.3 & 38.6 & 38.8 & 32.4 & 43.0 & 52.3 & 22.1 \\
\hline Food industry & 84.3 & 162.0 & 83.3 & 106.6 & 54.2 & 46.9 & 54.8 & 62.8 & 90.5 & 56.2 \\
\hline Other industry & 17.3 & 44.6 & 47.7 & 50.7 & 50.2 & 46.7 & 37.3 & 45.9 & 71.5 & 36.4 \\
\hline Agriculture & 17.0 & 27.7 & 34.6 & 18.6 & 31.4 & 40.9 & 14.2 & 17.4 & 12.9 & 7.4 \\
\hline \multirow[t]{2}{*}{ Other } & 0.0 & 0.0 & 0.0 & 0.0 & 0.0 & 0.0 & 0.0 & 0.0 & 0.0 & 0.0 \\
\hline & \multicolumn{10}{|c|}{ (In percent of total) } \\
\hline Total imports & 100.0 & 100.0 & 100.0 & 100.0 & 100.0 & 100.0 & 100.0 & 100.0 & 100.0 & 100.0 \\
\hline Industry & 96.7 & 96.7 & 95.1 & 97.8 & 94.8 & 92.6 & 97.0 & 97.0 & 98.2 & 98.3 \\
\hline Electric energy & 1.6 & 3.2 & 3.4 & 0.9 & 0.5 & 1.4 & 2.1 & 1.7 & 0.1 & 0.0 \\
\hline Oil and gas industry & 31.1 & 22.4 & 24.8 & 21.6 & 16.5 & 20.0 & 22.6 & 22.1 & 22.2 & 25.9 \\
\hline Coal industry & 3.3 & 3.0 & 1.0 & 2.1 & 3.3 & 2.0 & 1.3 & 2.3 & 3.0 & 1.1 \\
\hline Ferrous metallurgy & 3.4 & 1.8 & 1.4 & 2.6 & 1.6 & 2.0 & 2.0 & 2.5 & 2.7 & 2.6 \\
\hline Nonferrous metallurgy & 2.1 & 1.0 & 3.2 & 3.6 & 3.8 & 2.2 & 5.1 & 4.7 & 2.4 & 3.8 \\
\hline Chemical and petrochemical & 5.8 & 6.1 & 8.4 & 9.0 & 6.5 & 8.7 & 9.5 & 12.5 & 10.3 & 10.2 \\
\hline Machine building & 19.8 & 27.5 & 21.7 & 26.1 & 33.8 & 26.1 & 21.0 & 18.1 & 20.1 & 20.1 \\
\hline Lumber and paper & 3.8 & 3.1 & 4.1 & 4.2 & 3.2 & 4.8 & 4.5 & 3.8 & 4.8 & 4.7 \\
\hline Industrial construction materials & 1.9 & 1.9 & 1.9 & 1.9 & 1.8 & 1.6 & 2.1 & 3.5 & 2.7 & 2.7 \\
\hline Light industry & 4.4 & 2.0 & 6.8 & 7.2 & 6.4 & 7.0 & 6.9 & 7.3 & 7.3 & 5.2 \\
\hline Food industry & 16.1 & 19.3 & 11.7 & 12.7 & 9.0 & 8.5 & 11.7 & 10.7 & 12.6 & 13.3 \\
\hline Other industry & 3.3 & 5.3 & 6.7 & 6.0 & 8.4 & 8.4 & 8.0 & 7.8 & 10.0 & 8.6 \\
\hline Agriculture & 3.3 & 3.3 & 4.9 & 2.2 & 5.2 & 7.4 & 3.0 & 3.0 & 1.8 & 1.7 \\
\hline Other & 0.0 & 0.0 & 0.0 & 0.0 & 0.0 & 0.0 & 0.0 & 0.0 & 0.0 & 0.0 \\
\hline
\end{tabular}

Sources: Kyrgyz authorities; and Fund staff estimates.

1/ Import data are recorded on a CIF basis and do not incorporate estimates of unrecorded imports. 
Table 19. Kyrgyz Republic: Exports of Goods to CIS and Non-CIS Countries, 1995-2004

(In millions of U.S. dollars)

\begin{tabular}{|c|c|c|c|c|c|c|c|c|c|c|}
\hline & 1995 & 1996 & 1997 & 1998 & 1999 & 2000 & 2001 & 2002 & 2003 & $\begin{array}{r}2004 \\
\text { first half }\end{array}$ \\
\hline Total exports & 408.9 & 505.4 & 603.8 & 513.6 & 453.8 & 504.5 & 476.2 & 485.5 & 581.7 & 338.3 \\
\hline Exports to CIS countries 1/ & 269.2 & 393.4 & 319.3 & 230.6 & 183.3 & 207.4 & 168.5 & 168.8 & 201.4 & 116.4 \\
\hline Industry & 231.1 & 345.7 & 285.7 & 187.7 & 135.6 & 171.8 & 137.8 & 139.1 & 181.3 & 105.4 \\
\hline Electric energy & 41.0 & 73.6 & 83.2 & 25.6 & 52.0 & 79.8 & 46.8 & 22.0 & 19.2 & 6.2 \\
\hline Oil and gas industry & 1.5 & 1.9 & 1.5 & 2.2 & 0.9 & 1.9 & 4.4 & 5.7 & 6.6 & 2.3 \\
\hline Coal industry & 3.1 & 2.0 & 1.8 & 0.5 & 0.2 & 0.3 & 0.3 & 0.3 & 0.2 & 0.1 \\
\hline Ferrous metallurgy & 3.0 & 4.5 & 1.3 & 1.7 & 0.3 & 0.5 & 0.7 & 0.5 & 0.6 & 1.9 \\
\hline Nonferrous metallurgy & 11.9 & 22.6 & 10.4 & 6.6 & 2.5 & 1.3 & 2.3 & 2.1 & 1.4 & 0.3 \\
\hline Chemical and petrochemical & 9.1 & 7.9 & 9.6 & 5.2 & 5.5 & 8.3 & 5.4 & 7.1 & 9.3 & 6.4 \\
\hline Machine building & 39.5 & 48.6 & 49.8 & 53.7 & 33.5 & 39.6 & 36.4 & 32.5 & 36.6 & 18.4 \\
\hline Lumber and paper & 1.3 & 2.2 & 2.8 & 4.0 & 0.5 & 0.6 & 0.4 & 1.0 & 2.1 & 1.6 \\
\hline Industrial construction materials & 11.4 & 21.5 & 26.7 & 24.0 & 8.1 & 8.4 & 8.3 & 12.5 & 32.6 & 20.6 \\
\hline Light industry & 28.2 & 38.4 & 22.7 & 14.8 & 14.0 & 18.8 & 18.3 & 38.8 & 49.9 & 25.7 \\
\hline Food industry & 74.2 & 117.7 & 72.1 & 45.3 & 15.6 & 8.6 & 10.5 & 12.7 & 19.9 & 19.6 \\
\hline Other industry & 7.0 & 4.9 & 3.9 & 4.2 & 2.5 & 3.8 & 4.0 & 3.8 & 3.0 & 2.2 \\
\hline Agriculture & 38.2 & 47.7 & 33.5 & 42.9 & 47.5 & 35.7 & 30.7 & 29.7 & 20.1 & 11.0 \\
\hline Other & 0.0 & 0.0 & 0.0 & 0.0 & 0.2 & 0.0 & 0.0 & 0.0 & 0.0 & 0.0 \\
\hline Exports to non-CIS countries & 139.7 & 112.0 & 284.5 & 283.1 & 270.5 & 297.1 & 307.6 & 316.8 & 380.3 & 221.9 \\
\hline Industry & 135.0 & 96.4 & 272.9 & 273.4 & 261.3 & 285.8 & 293.0 & 290.2 & 369.8 & 216.1 \\
\hline Ferrous metallurgy & 7.4 & 2.4 & 1.7 & 1.2 & 1.3 & 4.9 & 5.3 & 4.7 & 5.8 & 7.7 \\
\hline Nonferrous metallurgy & 50.7 & 34.1 & 205.9 & 214.4 & 214.8 & 232.6 & 243.7 & 195.1 & 277.4 & 167.6 \\
\hline Chemical and petrochemical & 11.2 & 5.8 & 5.2 & 3.4 & 1.4 & 4.1 & 1.4 & 6.9 & 6.5 & 2.6 \\
\hline Machine building & 5.0 & 7.6 & 11.8 & 16.4 & 13.3 & 11.1 & 20.9 & 21.1 & 8.5 & 4.6 \\
\hline Lumber and paper & 0.6 & 0.2 & 0.7 & 0.6 & 0.3 & 0.4 & 0.2 & 0.3 & 0.3 & 0.1 \\
\hline Industrial construction materials & 0.2 & 0.3 & 0.2 & 0.0 & 0.1 & 0.1 & 0.0 & 0.2 & 0.7 & 0.2 \\
\hline Light industry & 54.3 & 36.1 & 38.0 & 26.0 & 18.3 & 25.1 & 13.8 & 26.0 & 24.0 & 9.1 \\
\hline Food industry & 4.9 & 9.4 & 7.5 & 9.2 & 3.5 & 5.0 & 2.0 & 4.4 & 4.7 & 2.6 \\
\hline Other industry & 0.6 & 0.6 & 2.0 & 2.1 & 8.4 & 2.5 & 5.8 & 31.6 & 42.1 & 21.6 \\
\hline Agriculture & 4.7 & 15.6 & 11.7 & 9.7 & 9.3 & 11.2 & 14.5 & 26.4 & 10.4 & 5.8 \\
\hline Other & 0.0 & 0.0 & 0.0 & 0.0 & 0.0 & 0.1 & 0.1 & 0.2 & 0.1 & 0.1 \\
\hline \multicolumn{11}{|l|}{ Memorandum items: } \\
\hline Exports associted with "shuttle trade" & $\ldots$ & $\ldots$ & 58.4 & 42.2 & 21.9 & 20.3 & 28.1 & 23.4 & 22.9 & 9.4 \\
\hline Included in official statistics & $\ldots$ & $\ldots$ & 31.4 & 20.8 & 13.2 & 13.9 & 24.0 & 21.6 & 21.9 & 9.2 \\
\hline Estimated (added to official statistics) & $\ldots$ & $\ldots$ & 27.0 & 21.4 & 8.7 & 6.4 & 4.1 & 1.8 & 1.0 & 0.2 \\
\hline
\end{tabular}

Sources: Kyrgyz authorities; and Fund staff estimates.

1/ Data for 1995 and 1996 do not include any estimates of unrecorded exports to other CIS countries. due to incomplete coverage. 
Table 20. Kyrgyz Republic: Imports of Goods from CIS and Non-CIS Countries, 1995-2004

(In millions of U.S. dollars)

\begin{tabular}{|c|c|c|c|c|c|c|c|c|c|c|}
\hline & 1995 & 1996 & 1997 & 1998 & 1999 & 2000 & 2001 & 2002 & 2003 & $\begin{array}{r}2004 \\
\text { first half }\end{array}$ \\
\hline Total imports & 522.3 & 837.7 & 709.3 & 841.5 & 599.7 & 554.6 & 467.2 & 586.8 & 717.0 & 421.8 \\
\hline Imports from CIS (c.i.f.) 1/ & 353.6 & 486.9 & 435.8 & 440.7 & 259.3 & 299.0 & 257.0 & 322.6 & 410.5 & 256.8 \\
\hline Industry & 349.9 & 473.6 & 423.0 & 428.6 & 248.8 & 283.9 & 245.2 & 307.7 & 401.9 & 251.5 \\
\hline Electric energy & 8.6 & 26.7 & 23.8 & 7.9 & 2.8 & 7.6 & 9.8 & 9.7 & 0.5 & 0.0 \\
\hline Oil and gas industry & 160.0 & 183.1 & 172.7 & 176.1 & 96.8 & 107.0 & 102.4 & 124.6 & 154.3 & 106.8 \\
\hline Coal industry & 17.0 & 25.1 & 6.9 & 17.4 & 19.6 & 10.8 & 6.2 & 13.6 & 21.4 & 4.6 \\
\hline Ferrous metallurgy & 16.3 & 14.3 & 9.1 & 17.0 & 6.0 & 10.2 & 8.5 & 10.4 & 17.6 & 10.3 \\
\hline Nonferrous metallurgy & 10.1 & 7.1 & 16.9 & 25.7 & 12.8 & 5.4 & 11.2 & 16.3 & 8.7 & 13.1 \\
\hline Chemical and petrochemical & 22.7 & 40.3 & 41.3 & 46.8 & 19.0 & 27.0 & 18.6 & 27.8 & 40.0 & 21.6 \\
\hline Machine building & 46.6 & 69.3 & 58.0 & 56.9 & 39.4 & 46.8 & 30.8 & 30.9 & 43.7 & 22.6 \\
\hline Lumber and paper & 15.8 & 18.6 & 18.8 & 21.2 & 10.0 & 15.3 & 12.1 & 13.8 & 21.2 & 13.0 \\
\hline Industrial construction materials & 8.2 & 10.9 & 10.6 & 11.5 & 7.6 & 6.5 & 7.6 & 9.0 & 14.6 & 8.9 \\
\hline Light industry & 16.0 & 10.7 & 11.7 & 5.6 & 3.0 & 2.0 & 1.6 & 1.5 & 2.7 & 1.6 \\
\hline Food industry & 22.2 & 57.3 & 41.9 & 21.2 & 25.6 & 30.0 & 29.2 & 42.3 & 62.3 & 40.0 \\
\hline Other industry & 6.4 & 10.1 & 11.2 & 21.3 & 6.1 & 15.3 & 7.2 & 7.7 & 14.9 & 9.0 \\
\hline Agriculture & 3.7 & 13.2 & 12.8 & 12.0 & 10.5 & 15.2 & 11.8 & 14.9 & 8.6 & 5.2 \\
\hline Other & 0.0 & 0.0 & 0.0 & 0.0 & 0.0 & 0.0 & 0.0 & 0.0 & 0.0 & 0.0 \\
\hline Imports from non-CIS (c.i.f.) 1/ & 168.8 & 350.8 & 273.5 & 400.8 & 340.5 & 255.6 & 210.3 & 264.2 & 306.5 & 165.1 \\
\hline Industry & 155.5 & 336.3 & 251.7 & 394.2 & 319.5 & 229.9 & 207.9 & 261.7 & 302.2 & 162.9 \\
\hline Electric energy & 0.0 & 0.0 & 0.0 & 0.0 & 0.0 & 0.0 & 0.0 & 0.0 & 0.0 & 0.0 \\
\hline Oil and gas industry & 2.6 & 4.5 & 3.2 & 5.4 & 2.3 & 3.8 & 3.1 & 4.9 & 4.7 & 2.5 \\
\hline Coal industry & 0.0 & 0.0 & 0.1 & 0.0 & 0.0 & 0.0 & 0.0 & 0.0 & 0.0 & 0.0 \\
\hline Ferrous metallurgy & 1.5 & 0.7 & 0.8 & 4.6 & 3.5 & 0.8 & 1.1 & 4.1 & 1.8 & 0.7 \\
\hline Nonferrous metallurgy & 1.1 & 1.6 & 5.5 & 4.9 & 10.1 & 7.1 & 12.8 & 11.4 & 8.9 & 3.0 \\
\hline Chemical and petrochemical & 7.3 & 10.7 & 18.6 & 28.7 & 19.7 & 21.1 & 25.8 & 45.5 & 33.5 & 21.4 \\
\hline Machine building & 57.0 & 161.2 & 96.0 & 162.4 & 163.0 & 98.2 & 67.4 & 75.6 & 100.6 & 62.1 \\
\hline Lumber and paper & 4.0 & 7.6 & 10.4 & 14.1 & 9.1 & 11.5 & 8.9 & 8.5 & 13.5 & 6.7 \\
\hline Industrial construction materials & 1.9 & 5.0 & 2.7 & 4.5 & 3.4 & 2.3 & 2.4 & 11.5 & 4.9 & 2.4 \\
\hline Light industry & 7.2 & 5.9 & 36.6 & 54.7 & 35.7 & 36.8 & 30.8 & 41.5 & 49.6 & 20.4 \\
\hline Food industry & 62.0 & 104.7 & 41.4 & 85.4 & 28.7 & 16.9 & 25.6 & 20.5 & 28.2 & 16.2 \\
\hline Other industry & 10.9 & 34.5 & 36.5 & 29.4 & 44.1 & 31.4 & 30.2 & 38.2 & 56.6 & 27.4 \\
\hline Agriculture & 13.3 & 14.5 & 21.8 & 6.6 & 21.0 & 25.7 & 2.4 & 2.5 & 4.3 & 2.1 \\
\hline \multicolumn{11}{|l|}{ Memorandum items: } \\
\hline Imports associted with "shuttle trade" & $\ldots$ & $\ldots$ & 82.4 & 100.5 & 58.3 & 56.7 & 50.9 & 59.5 & 80.4 & 39.4 \\
\hline Included in official statistics & $\ldots$ & $\ldots$ & 59.4 & 72.7 & 44.8 & 52.7 & 46.6 & 56.6 & 75.6 & 38.3 \\
\hline Estimated (added to official statistics) & $\ldots$ & $\ldots$ & 23.0 & 27.8 & 13.5 & 3.9 & 4.3 & 2.9 & 4.8 & 1.1 \\
\hline
\end{tabular}

Sources: Kyrgyz authorities; and Fund staff estimates.

1/ Import data are recorded on a CIF basis and do not incorporate estimates of unrecorded imports. 
Table 21. Kyrgyz Republic: Direction of Trade, 1995-2004

(In millions of U.S. dollars)

\begin{tabular}{|c|c|c|c|c|c|c|c|c|c|c|}
\hline & 1995 & 1996 & 1997 & 1998 & 1999 & 2000 & 2001 & 2002 & 2003 & $\begin{array}{r}2004 \\
\text { first half }\end{array}$ \\
\hline & \multicolumn{10}{|c|}{ Exports } \\
\hline Total non-CIS & 139.7 & 112.0 & 284.5 & 283.1 & 270.5 & 297.1 & 307.6 & 316.8 & 380.3 & 221.9 \\
\hline 9156 China & 68.5 & 36.4 & 31.6 & 15.7 & 25.3 & 44.1 & 19.4 & 41.1 & 23.3 & 19.6 \\
\hline 9826 United Kingdom & 27.4 & 5.3 & 1.4 & 1.6 & 12.4 & 18.8 & 14.1 & 0.9 & 0.1 & 0.1 \\
\hline 9840 United States & 4.0 & 17.6 & 17.9 & 7.6 & 11.2 & 2.8 & 7.1 & 36.1 & 6.5 & 1.3 \\
\hline 9250 France & 3.6 & 1.8 & 0.3 & 7.0 & 8.2 & 3.3 & 1.8 & 5.6 & 0.2 & 0.2 \\
\hline 9792 Turkey & 3.2 & 5.3 & 8.0 & 7.4 & 4.6 & 7.2 & 13.8 & 16.4 & 11.0 & 6.2 \\
\hline 9380 Italy & 2.9 & 2.5 & 2.6 & 2.6 & 0.3 & 1.0 & 1.4 & 1.0 & 0.4 & 0.0 \\
\hline 9276 Germany & 2.1 & 2.9 & 18.1 & 192.2 & 148.2 & 144.6 & 94.4 & 1.8 & 3.0 & 1.0 \\
\hline 9616 Poland & 1.3 & 0.5 & 1.4 & 1.0 & 0.5 & 0.9 & 0.4 & 0.3 & 0.2 & 0.2 \\
\hline 9756 Switzerland & 1.8 & 0.9 & 162.3 & 1.1 & 18.1 & 34.1 & 124.2 & 96.4 & 117.9 & 56.8 \\
\hline Other & 24.9 & 38.8 & 40.9 & 47.0 & 41.7 & 40.3 & 31.1 & 117.4 & 217.7 & 136.6 \\
\hline Total CIS & 269.2 & 393.4 & 319.3 & 230.6 & 183.3 & 207.4 & 168.5 & 168.8 & 201.4 & 116.5 \\
\hline 3700 Armenia & 0.0 & 0.0 & 0.0 & 0.0 & 60.1 & 0.9 & 0.0 & 0.1 & 0.0 & 0.0 \\
\hline 2400 Azerbaijan & 2.1 & 3.2 & 2.8 & 2.6 & 1.5 & 4.0 & 2.1 & 5.6 & 2.0 & 0.8 \\
\hline 1500 Belarus & 5.0 & 5.6 & 8.6 & 5.3 & 4.9 & 3.0 & 3.2 & 1.3 & 1.5 & 0.8 \\
\hline 2200 Georgia & 0.7 & 0.1 & 0.6 & 0.5 & 0.4 & 0.2 & 0.2 & 0.1 & 0.3 & 0.2 \\
\hline 1900 Kazakhstan & 66.8 & 112.5 & 87.1 & 85.5 & 45.0 & 33.4 & 39.0 & 36.8 & 57.1 & 36.5 \\
\hline 2800 Moldova & 1.0 & 0.8 & 0.0 & 0.2 & 0.5 & 0.0 & 0.3 & 0.1 & 0.5 & 0.1 \\
\hline 1100 Russia & 104.8 & 134.6 & 98.8 & 83.7 & 70.7 & 65.1 & 64.5 & 80.0 & 97.0 & 57.4 \\
\hline 3500 Tajikistan & 8.3 & 8.3 & 12.7 & 8.3 & 9.5 & 7.5 & 6.7 & 10.2 & 18.9 & 9.6 \\
\hline 3900 Turkmenistan & 2.2 & 3.2 & 2.6 & 1.2 & 2.8 & 2.7 & 1.5 & 2.4 & 2.3 & 1.6 \\
\hline 1300 Ukraine & 8.3 & 9.4 & 4.6 & 4.7 & 1.5 & 1.1 & 2.9 & 4.3 & 5.4 & 3.0 \\
\hline 1700 Uzbekistan & 70.0 & 115.8 & 101.5 & 38.5 & 46.6 & 89.4 & 48.0 & 27.8 & 16.3 & 6.6 \\
\hline \multirow[t]{2}{*}{ Total exports } & 408.9 & 505.4 & 603.8 & 513.6 & 453.8 & 504.5 & 476.2 & 485.5 & 581.7 & 338.3 \\
\hline & \multicolumn{10}{|c|}{ Imports } \\
\hline Total non-CIS & 168.8 & 350.8 & 273.5 & 400.8 & 340.5 & 255.6 & 210.3 & 264.2 & 306.5 & 165.1 \\
\hline 9792 Turkey & 38.5 & 47.6 & 43.7 & 37.4 & 23.1 & 26.8 & 15.8 & 17.0 & 26.0 & 11.8 \\
\hline $9192 \mathrm{Cuba}$ & 22.7 & 22.1 & 0.0 & 12.1 & 4.1 & 0.0 & 0.1 & 0.0 & 0.0 & 0.0 \\
\hline 9840 United States & 19.1 & 35.7 & 39.6 & 40.9 & 54.2 & 53.8 & 26.8 & 47.4 & 47.9 & 27.3 \\
\hline 9276 Germany & 18.8 & 31.8 & 38.4 & 53.1 & 47.3 & 25.2 & 24.3 & 31.4 & 38.2 & 22.2 \\
\hline 9392 Japan & 7.2 & 12.5 & 2.7 & 4.3 & 12.0 & 10.3 & 5.8 & 6.4 & 11.8 & 5.5 \\
\hline 9156 China & 6.3 & 7.8 & 32.5 & 44.4 & 36.9 & 36.9 & 48.5 & 59.0 & 77.7 & 37.2 \\
\hline 9124 Canada & 5.9 & 42.5 & 5.2 & 14.4 & 25.4 & 11.3 & 10.9 & 9.0 & 8.3 & 5.1 \\
\hline Other & 50.3 & 150.8 & 111.4 & 194.3 & 137.4 & 91.3 & 78.1 & 93.9 & 96.5 & 56.0 \\
\hline Total CIS & 353.6 & 486.9 & 435.8 & 440.7 & 259.3 & 299.0 & 257.0 & 322.5 & 410.5 & 256.7 \\
\hline 3700 Armenia & 0.6 & 0.0 & 0.3 & 0.4 & 0.0 & 0.0 & 0.0 & 0.4 & 0.0 & 0.0 \\
\hline 2400 Azerbaijan & 3.3 & 1.4 & 2.5 & 7.2 & 3.4 & 2.4 & 0.4 & 2.4 & 0.4 & 0.0 \\
\hline 1500 Belarus & 5.0 & 6.1 & 10.3 & 9.6 & 5.3 & 3.9 & 6.0 & 5.1 & 5.9 & 1.5 \\
\hline 2200 Georgia & 0.3 & 1.6 & 3.1 & 0.3 & 0.2 & 0.1 & 0.0 & 0.1 & 0.5 & 0.2 \\
\hline 1900 Kazakhstan & 112.5 & 139.5 & 69.6 & 75.3 & 72.7 & 57.4 & 81.8 & 123.9 & 170.9 & 97.1 \\
\hline 2800 Moldova & 0.2 & 0.2 & 0.4 & 0.0 & 0.2 & 0.1 & 0.3 & 0.7 & 1.4 & 0.5 \\
\hline 1100 Russia & 114.3 & 174.5 & 190.8 & 204.1 & 109.4 & 132.6 & 85.1 & 116.7 & 176.1 & 121.2 \\
\hline 3500 Tajikistan & 4.8 & 6.3 & 10.0 & 6.4 & 4.0 & 1.9 & 1.5 & 3.5 & 3.1 & 1.0 \\
\hline 3900 Turkmenistan & 18.6 & 13.6 & 15.5 & 8.2 & 7.8 & 18.7 & 9.0 & 1.7 & 0.4 & 0.4 \\
\hline 1300 Ukraine & 4.9 & 12.3 & 4.8 & 6.9 & 6.3 & 7.0 & 6.2 & 7.8 & 12.6 & 9.2 \\
\hline 1700 Uzbekistan & 88.9 & 131.5 & 128.6 & 122.2 & 50.0 & 75.1 & 66.7 & 60.1 & 39.2 & 25.5 \\
\hline Total imports & 522.3 & 837.7 & 709.3 & 841.5 & 599.7 & 554.6 & 467.2 & 586.7 & 717.0 & 421.7 \\
\hline
\end{tabular}

Source: Kyrgyz authorities. 


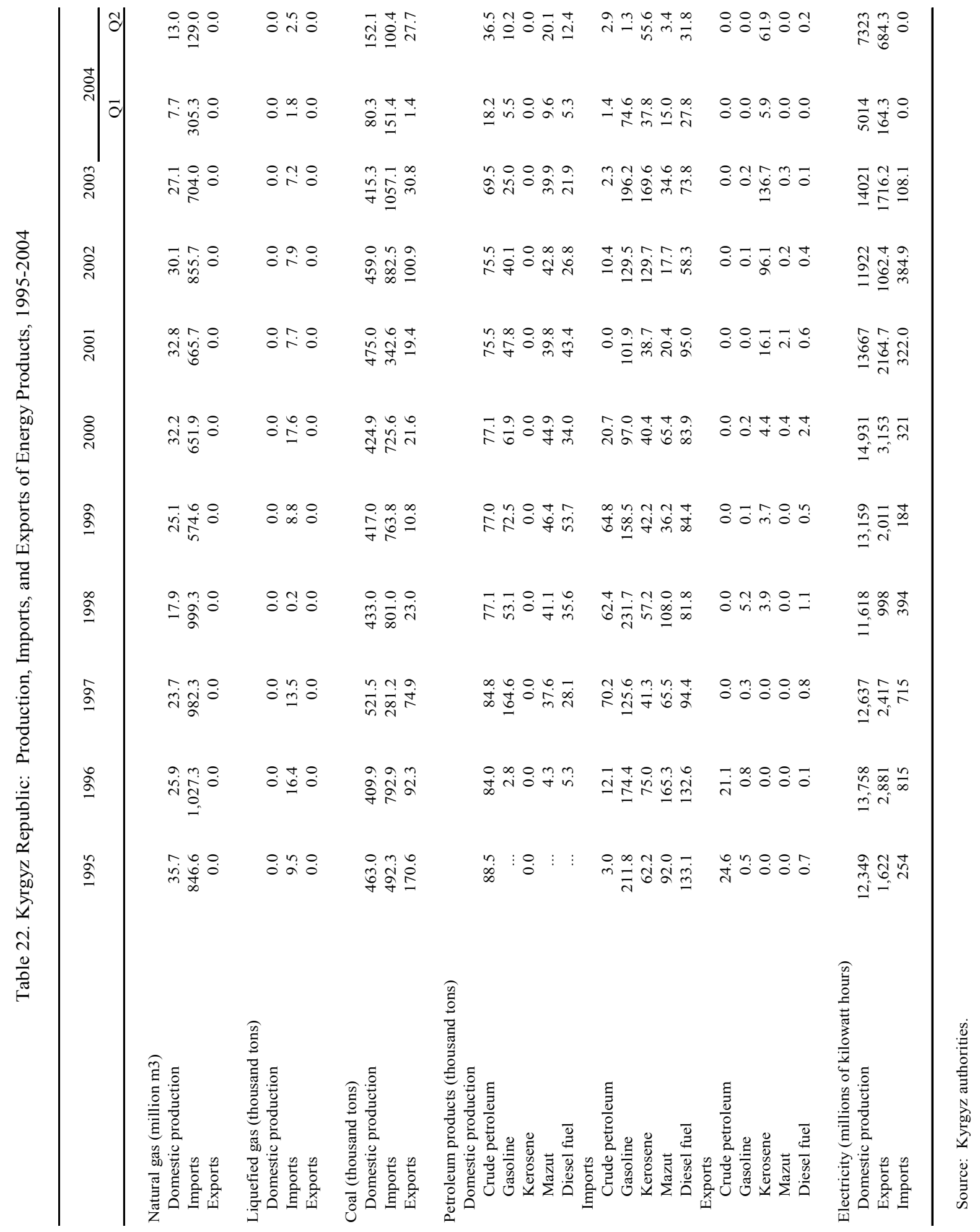


Table 23. Kyrgyz Republic: Foreign Direct Investment by Country of Origin, 1995-2003 1/ (In millions of U.S. dollars)

\begin{tabular}{|c|c|c|c|c|c|c|c|c|c|}
\hline & 1995 & 1996 & 1997 & 1998 & 1999 & 2000 & 2001 & 2002 & 2003 \\
\hline Total direct foreign investment & 96.1 & 46.8 & 83.0 & 109.2 & 44.4 & -2.4 & 5.0 & 4.7 & 45.5 \\
\hline CIS countries & 0.3 & 1.3 & 2.8 & 2.5 & -1.1 & 1.3 & -1.7 & 16.3 & 4.7 \\
\hline Belarus & 0.0 & 0.0 & 0.0 & 0.0 & 0.0 & 0.3 & -0.1 & -0.2 & 0.0 \\
\hline Kazakhstan & 0.0 & 0.3 & 1.4 & 2.4 & -0.7 & -0.1 & -0.2 & 3.4 & 0.2 \\
\hline Russia & 0.2 & 0.8 & 1.2 & -0.6 & -0.7 & 1.0 & 0.5 & 11.8 & 3.4 \\
\hline Ukraine & 0.0 & 0.0 & 0.0 & 0.0 & 0.0 & 0.0 & -1.4 & -0.1 & 0.3 \\
\hline Uzbekistan & 0.0 & 0.2 & 0.2 & 0.6 & -0.2 & -0.2 & -0.8 & 0.0 & 0.1 \\
\hline Others & 0.0 & 0.0 & 0.0 & 0.2 & 0.5 & 0.3 & 0.3 & 1.4 & 0.7 \\
\hline Non-CIS countries & 95.8 & 45.5 & 80.3 & 106.7 & 45.5 & -3.7 & 6.7 & -11.4 & 40.8 \\
\hline United States & -0.1 & 4.4 & 6.0 & 25.5 & 7.0 & 1.5 & -3.4 & 3.9 & -7.2 \\
\hline Canada & 92.5 & 19.2 & 31.3 & 1.7 & 4.2 & -5.9 & -10.1 & -9.6 & 19.4 \\
\hline $\begin{array}{l}\text { European Union 2/ } \\
\text { of which: }\end{array}$ & 0.5 & 3.8 & 18.5 & 30.7 & 26.3 & 4.3 & 18.1 & -10.0 & -3.8 \\
\hline Germany & 0.2 & 0.7 & 4.4 & 0.9 & 12.1 & 8.2 & 18.4 & -3.5 & -5.7 \\
\hline United Kingdom & 0.0 & 0.6 & 10.2 & 20.7 & 11.5 & -3.8 & -1.5 & -1.7 & -2.0 \\
\hline Italy & 0.0 & 0.8 & 3.0 & 4.5 & 2.5 & -7.5 & 0.0 & -6.2 & -0.1 \\
\hline Switzerland & 2.0 & 4.3 & 0.9 & 3.1 & -1.7 & -0.7 & -4.1 & 0.0 & -0.9 \\
\hline Turkey & 0.1 & 10.9 & 16.2 & 10.5 & 12.7 & -3.3 & 0.4 & 4.1 & 17.6 \\
\hline Japan & 0.0 & 0.2 & 0.5 & 10.2 & 0.2 & -0.1 & 0.0 & 0.0 & -0.3 \\
\hline India & 0.0 & 0.0 & 1.1 & 3.2 & -1.5 & -0.8 & 0.6 & 0.3 & 0.1 \\
\hline Pakistan & 0.0 & 0.0 & 0.3 & 0.4 & 0.1 & -0.2 & 0.0 & -2.5 & -0.1 \\
\hline Malaysia & 0.0 & 0.1 & 2.1 & 1.7 & 0.1 & 3.0 & 0.1 & 0.0 & 0.0 \\
\hline Others & 0.7 & 2.4 & 3.5 & 19.9 & -1.9 & -1.4 & 5.1 & 2.4 & 16.0 \\
\hline
\end{tabular}

Source: Kyrgyz authorities.

1/ Flows of FDI from nonresidents to the Kyrgyz Republic (excludes FDI by Kyrgyz residents abroad). 2/ 25 members of EU 
Table 24. Kyrgyz Republic: External Public Debt and Debt Service, 1995-2004 1/

(in millions of US dollars, end-period stock)

\begin{tabular}{|c|c|c|c|c|c|c|c|c|c|c|c|}
\hline & \multirow[t]{2}{*}{1995} & \multirow[t]{2}{*}{1996} & \multirow[t]{2}{*}{1997} & \multirow[t]{2}{*}{1998} & \multirow[t]{2}{*}{1999} & \multirow[t]{2}{*}{2000} & \multirow[t]{2}{*}{2001} & \multirow[t]{2}{*}{2002} & \multirow[t]{2}{*}{2003} & \multicolumn{2}{|c|}{2004} \\
\hline & & & & & & & & & & Q1 & $\mathrm{Q} 2$ \\
\hline \multicolumn{12}{|l|}{ External public debt } \\
\hline Debt outstanding & 594.1 & 742.0 & 904.9 & $1,177.5$ & $1,358.6$ & $1,520.3$ & $1,441.5$ & $1,578.7$ & $1,789.8$ & $1,798.4$ & $1,802.4$ \\
\hline Multilateral & 300.9 & 426.6 & 584.3 & 743.2 & 848.2 & 942.8 & 947.1 & $1,068.4$ & $1,222.4$ & $1,223.6$ & $1,222.1$ \\
\hline Concessional & 234.0 & 343.3 & 487.4 & 654.4 & 748.4 & 848.2 & 873.0 & $1,007.8$ & $1,177.1$ & $1,179.2$ & $1,180.7$ \\
\hline IDA & 139.6 & 193.5 & 251.2 & 328.4 & 342.5 & 406.8 & 388.4 & 453.2 & 529.1 & 524.8 & 530.2 \\
\hline PRGF & 60.4 & 83.4 & 127.2 & 139.1 & 159.7 & 163.6 & 167.5 & 178.6 & 201.9 & 208.2 & 198.7 \\
\hline Others & 34.0 & 66.4 & 109.0 & 186.9 & 246.2 & 277.8 & 317.1 & 376.0 & 446.1 & 446.2 & 451.8 \\
\hline Non-concessional & 66.9 & 83.3 & 96.9 & 88.8 & 99.8 & 94.7 & 74.1 & 60.6 & 45.2 & 44.4 & 41.4 \\
\hline IMF & 66.6 & 57.3 & 49.2 & 37.8 & 30.7 & 24.4 & 11.8 & 5.4 & - & - & - \\
\hline Others & 0.3 & 26.0 & 47.7 & 51.0 & 69.1 & 70.3 & 62.3 & 55.2 & 45.2 & 44.4 & 41.4 \\
\hline Bilateral & 293.2 & 315.4 & 320.7 & 434.3 & 510.4 & 577.5 & 494.4 & 510.3 & 567.5 & 574.8 & 580.3 \\
\hline CIS (non-concessional) & 176.9 & 150.0 & 180.5 & 208.2 & 207.8 & 160.9 & 178.1 & 182.7 & 187.6 & 187.9 & 190.4 \\
\hline Non-CIS & 116.3 & 165.4 & 140.2 & 226.0 & 302.6 & 416.6 & 316.3 & 327.5 & 379.9 & 386.9 & 390.0 \\
\hline Concessional & 78.9 & 113.6 & 96.8 & 133.3 & 211.1 & 247.6 & 255.3 & 262.6 & 312.9 & 316.3 & 317.6 \\
\hline Non-concessional & 37.4 & 51.8 & 43.4 & 92.7 & 91.5 & 169.0 & 61.0 & 65.0 & 67.0 & 70.7 & 72.4 \\
\hline \multicolumn{12}{|l|}{ Disbursement } \\
\hline Disbursements & 207.7 & 180.0 & 175.6 & 170.2 & 232.3 & 148.0 & 125.1 & 124.8 & 143.8 & 27.2 & 34.4 \\
\hline Multilateral & 162.7 & 134.2 & 164.4 & 137.8 & 159.1 & 88.4 & 105.1 & 87.0 & 90.8 & 21.6 & 14.0 \\
\hline Concessional & 162.7 & 117.4 & 162.6 & 130.7 & 136.8 & 80.7 & 104.7 & 86.8 & 90.8 & 21.6 & 14.0 \\
\hline IDA & 82.4 & 57.5 & 65.4 & 68.1 & 21.2 & 34.4 & 26.1 & 33.4 & 29.3 & 1.9 & 7.0 \\
\hline PRGF & 46.3 & 23.5 & 43.8 & 14.2 & 26.7 & 18.7 & 14.9 & 15.4 & 29.5 & 14.0 & - \\
\hline Others & 34.0 & 36.4 & 53.5 & 48.3 & 88.9 & 27.6 & 63.7 & 38.0 & 32.0 & 5.8 & 7.0 \\
\hline Non-concessional & 0.0 & 16.8 & 1.7 & 7.1 & 22.2 & 7.7 & 0.5 & 0.1 & - & - & - \\
\hline IBRD & 0.0 & 0.0 & 0.0 & 0.0 & 0.0 & 0.0 & 0.0 & - & - & - & - \\
\hline IMF & 0.0 & 0.0 & 0.0 & 0.0 & 0.0 & 0.0 & 0.0 & - & - & - & - \\
\hline Others & 0.0 & 16.8 & 1.7 & 7.1 & 22.2 & 7.7 & 0.5 & 0.1 & - & - & - \\
\hline Bilateral & 45.0 & 45.8 & 11.2 & 32.5 & 73.3 & 59.5 & 20.0 & 37.8 & 53.0 & 5.6 & 20.4 \\
\hline CIS (non-concessional) & 0.0 & 0.0 & 6.0 & 6.3 & 2.7 & 21.2 & 0.0 & 15.3 & 25.5 & 4.2 & 8.8 \\
\hline Non-CIS & 45.0 & 45.8 & 5.2 & 26.2 & 70.6 & 38.4 & 20.0 & 22.5 & 27.6 & 1.4 & 11.6 \\
\hline Concessional & 37.9 & 31.2 & 5.2 & 26.2 & 70.6 & 38.4 & 16.6 & 18.6 & 21.7 & 1.4 & 7.6 \\
\hline Non-concessional & 7.1 & 14.6 & 0.0 & 0.0 & 0.0 & 0.0 & 3.3 & 3.9 & 5.9 & - & 4.0 \\
\hline Interest payments & 22.2 & 18.5 & 20.8 & 24.9 & 19.3 & 23.6 & 23.8 & 13.8 & 11.7 & 1.5 & 5.4 \\
\hline Multilateral & 6.4 & 5.2 & 9.8 & 11.7 & 12.8 & 13.3 & 12.4 & 10.1 & 10.4 & 1.5 & 4.5 \\
\hline IDA/IBRD & 0.6 & 1.4 & 1.8 & 1.9 & 2.4 & 2.6 & 2.9 & 3.0 & 3.6 & 0.7 & 1.7 \\
\hline IMF & 3.9 & 3.2 & 3.1 & 2.6 & 2.1 & 2.1 & 1.6 & 1.2 & 1.0 & 0.0 & 0.5 \\
\hline Others & 1.9 & 0.6 & 4.9 & 7.2 & 8.3 & 8.7 & 8.0 & 5.9 & 5.8 & 0.8 & 2.4 \\
\hline Bilateral & 15.8 & 13.3 & 9.4 & 13.9 & 9.2 & 10.3 & 11.3 & 3.7 & 1.3 & 0.0 & 0.8 \\
\hline CIS & 13.9 & 7.5 & 5.3 & 5.8 & 1.8 & 1.2 & 4.4 & 2.3 & 1.0 & 0.0 & 0.6 \\
\hline Non-CIS & 1.9 & 5.8 & 4.1 & 8.1 & 7.4 & 9.1 & 7.0 & 1.3 & 0.3 & 0.0 & 0.2 \\
\hline Amortization & 37.3 & 45.5 & 15.8 & 23.7 & 13.3 & 31.6 & 47.9 & 56.3 & 42.9 & 6.5 & 14.4 \\
\hline Multilateral & 0.0 & 3.9 & 11.7 & 15.2 & 11.8 & 25.1 & 26.0 & 32.7 & 41.8 & 6.5 & 13.5 \\
\hline IDA/IBRD & 0.0 & 0.0 & 0.0 & 0.0 & 0.0 & 0.0 & 0.0 & 0.0 & 0.6 & 0.0 & 0.6 \\
\hline IMF & 0.0 & 3.9 & 9.8 & 11.5 & 7.4 & 11.4 & 17.0 & 23.5 & 30.4 & 5.4 & 9.2 \\
\hline Others & 0.0 & 0.0 & 1.9 & 3.7 & 4.3 & 13.7 & 9.0 & 9.1 & 10.8 & 1.0 & 3.7 \\
\hline Bilateral & 37.3 & 41.6 & 4.1 & 8.5 & 1.5 & 6.5 & 21.9 & 23.7 & 1.1 & 0.0 & 0.9 \\
\hline CIS & 37.3 & 36.6 & 3.0 & 7.3 & 0.1 & 1.4 & 11.6 & 0.0 & 0.0 & 0.0 & 0.0 \\
\hline Non-CIS & 0.0 & 5.0 & 1.1 & 1.2 & 1.4 & 5.1 & 10.3 & 23.7 & 1.1 & 0.0 & 0.9 \\
\hline
\end{tabular}

Sources: Kyrgyz authorities.

1/ Includes only public and publicly guaranteed debt. 
Table 25a. Kyrgyz Republic: State Government Finances, 1995-2003

\begin{tabular}{|c|c|c|c|c|c|c|c|c|c|}
\hline & 1995 & 1996 & 1997 & 1998 & 1999 & 2000 & 2001 & 2002 & 2003 \\
\hline & \multicolumn{9}{|c|}{ (In millions of soms) } \\
\hline Total revenue and grants & 2,703 & 3,728 & 4,973 & 6,149 & 8,608 & 9,896 & 12,544 & 14,402 & 15,666 \\
\hline Total revenue & 2,648 & 3,527 & 4,778 & 5,953 & 7,612 & 9,287 & 11,922 & 13,569 & 15,249 \\
\hline Current revenue & 2,599 & 3,233 & 4,652 & 5,863 & 7,464 & 9,264 & 11,864 & 13,439 & 15,112 \\
\hline Tax revenue & 2,423 & 2,968 & 3,847 & 4,867 & 5,972 & 7,675 & 9,188 & 10,475 & 11,917 \\
\hline Income tax & 713 & 669 & 685 & 892 & 1,151 & 1,379 & 2,008 & 2,199 & 2,381 \\
\hline VAT & 705 & 1,248 & 1,728 & 1,966 & 2,253 & 2,976 & 4,221 & 4,794 & 5,526 \\
\hline Excises & 298 & 246 & 451 & 722 & 1,253 & 1,518 & 1,103 & 1,082 & 1,164 \\
\hline Customs & 134 & 206 & 245 & 381 & 307 & 275 & 301 & 419 & 423 \\
\hline Land tax & 73 & 122 & 278 & 291 & 199 & 179 & 209 & 318 & 312 \\
\hline Road tax and Emergency Fund & 217 & 239 & 319 & 422 & 566 & 1,059 & 990 & 1,030 & 1,173 \\
\hline Retail sales tax & 168 & 114 & 85 & 151 & 187 & 232 & 305 & 410 & 471 \\
\hline Other & 114 & 125 & 56 & 43 & 58 & 57 & 50 & 223 & 467 \\
\hline Nontax revenue & 176 & 265 & 805 & 997 & 1,491 & 1,588 & 2,677 & 2,964 & 3,195 \\
\hline Capital revenue & 49 & 293 & 126 & 90 & 148 & 24 & 57 & 130 & 137 \\
\hline Grants & 55 & 201 & 195 & 197 & 996 & 608 & 622 & 833 & 418 \\
\hline Total expenditure (excluding net lending) & 4,802 & 5,804 & 7,795 & 10,008 & 14,991 & 16,572 & 16,880 & 18,710 & 20,403 \\
\hline Current expenditure & 4,031 & 4,907 & 6,638 & 7,764 & 9,887 & 11,263 & 12,966 & 14,238 & 16,383 \\
\hline Wages and Social Fund contributions & 1,528 & 1,682 & 2,065 & 2,630 & 2,787 & 3,347 & 4,301 & 4,876 & 5,673 \\
\hline Transfers and subsidies & 1,008 & 994 & 952 & 1,036 & 1,049 & 1,628 & 2,221 & 2,558 & 2,689 \\
\hline Transfers to Social Fund & 202 & 397 & 564 & 504 & 489 & 372 & 398 & 840 & 1,122 \\
\hline Social benefits and pension supplements & 111 & 104 & 118 & 160 & 189 & 222 & 398 & 435 & 534 \\
\hline General transfer (subsidy) & 91 & 293 & 446 & 344 & 300 & 150 & 0 & 405 & 588 \\
\hline Interest & 68 & 284 & 520 & 715 & 1,436 & 1,507 & 1,228 & 1,238 & 1,280 \\
\hline Domestic & 9 & 81 & 242 & 292 & 233 & 344 & 264 & 255 & 367 \\
\hline Foreign & 59 & 203 & 278 & 423 & 1,203 & 1,163 & 964 & 984 & 913 \\
\hline Purchases of other goods and services & 1,226 & 1,549 & 2,538 & 2,878 & 4,126 & 4,409 & 4,818 & 4,726 & 5,618 \\
\hline Capital expenditure (including PIP) & 771 & 897 & 1,157 & 2,244 & 5,104 & 5,309 & 3,915 & 4,472 & 4,020 \\
\hline Domestically financed capital expenditure $2 /$ & 161 & 158 & 207 & 292 & 526 & 897 & 676 & 932 & 984 \\
\hline Foreign financed PIP & 610 & 739 & 949 & 1,952 & 4,578 & 4,412 & 3,239 & 3,541 & 3,036 \\
\hline Financial balance & $-2,099$ & $-2,076$ & $-2,822$ & $-3,859$ & $-6,383$ & $-6,676$ & $-4,337$ & $-4,308$ & $-4,736$ \\
\hline Net lending & 556 & 101 & -17 & -165 & -189 & -311 & -509 & -443 & -414 \\
\hline Accrual surplus $(+)$ / deficit (-) & $-2,655$ & $-2,177$ & $-2,805$ & $-3,694$ & $-6,194$ & $-6,366$ & $-3,828$ & $-3,865$ & $-4,323$ \\
\hline Net accumulation of expenditure arrears & -133 & -56 & -7 & 441 & -351 & -175 & 155 & -231 & -53 \\
\hline Rescheduling of external interest arrears 1 / & 0 & 0 & 0 & 11 & 728 & 509 & 0 & 0 & 0 \\
\hline Cash surplus (+) / deficit (-) & $-2,788$ & $-2,232$ & $-2,812$ & $-3,242$ & $-5,817$ & $-6,032$ & $-3,673$ & $-4,096$ & $-4,376$ \\
\hline Total financing & 2,788 & 2,232 & 2,812 & 3,242 & 5,817 & 6,032 & 3,673 & 4,096 & 4,376 \\
\hline External financing & 1,467 & 1,620 & 2,451 & 3,046 & 7,084 & 5,784 & 4,208 & 3,933 & 3,513 \\
\hline Public investment program (PIP) & 610 & 739 & 949 & 1,952 & 4,578 & 4,412 & 3,239 & 3,630 & 2,858 \\
\hline Disbursements (BOP support) & 1,293 & 885 & 1,477 & 1,123 & 2,560 & 1,604 & 2,007 & 356 & 218 \\
\hline Turkish loan & 143 & 102 & 115 & 0 & 0 & 0 & 0 & 0 & 0 \\
\hline Total amortization & -579 & -188 & -169 & -254 & -168 & $-1,621$ & $-1,833$ & -878 & $-1,474$ \\
\hline Arrears and rescheduling & $\ldots$ & 82 & 78 & 225 & 114 & 1,389 & 795 & 825 & 1,911 \\
\hline Domestic financing & 1,321 & 497 & 339 & 113 & $-1,385$ & 78 & -834 & 33 & 556 \\
\hline NBKR & 1,252 & 453 & 117 & 76 & $-1,015$ & -43 & -949 & -10 & 394 \\
\hline Commercial banks & 70 & 22 & 132 & 39 & -247 & 89 & 134 & 52 & 133 \\
\hline Nonbank & 0 & 22 & 91 & -2 & -123 & 33 & -19 & -9 & 29 \\
\hline Exceptional Financing & 0 & 116 & 22 & 84 & 118 & 170 & 299 & 130 & 307 \\
\hline \multicolumn{10}{|l|}{ Memorandum items: } \\
\hline Primary balance & $-2,720$ & $-1,948$ & $-2,292$ & $-2,527$ & $-4,381$ & $-4,525$ & $-2,446$ & $-2,858$ & $-3,095$ \\
\hline Domestic balance 2/ & $-2,120$ & $-1,290$ & $-1,584$ & -868 & -36 & -457 & 529 & 428 & -427 \\
\hline Underlying balance 3 / & $-2,165$ & $-1,410$ & $-1,557$ & -809 & -878 & -951 & -439 & -939 & $-1,403$ \\
\hline Socail Expenditure 4/ & 2,724 & 3,138 & 3,826 & 3,998 & 4,779 & 5,369 & 6,445 & 8,349 & 9,164 \\
\hline Subsidies to public enterprises & 165 & 200 & 153 & 212 & 323 & 444 & 574 & 737 & 635 \\
\hline
\end{tabular}

Sources: Kyrgyz authorities and Fund staff estimates.

1/ In 2000, includes interest rescheduled for bilateral loans.

2/ Primary balance excluding foreign interest payments and foreign loan financed PIP.

3/ Primary balance excluding revenues from gold projects, grant, and foreign loan financed PIP.

4/ Includes health, education, social security, social insurance, and housing. 
Table 25b. Kyrgyz Republic: State Government Finances, 1995-2003

\begin{tabular}{|c|c|c|c|c|c|c|c|c|c|}
\hline & 1995 & 1996 & 1997 & 1998 & 1999 & 2000 & 2001 & 2002 & 2003 \\
\hline & \multicolumn{9}{|c|}{ (In percent of GDP) } \\
\hline Total revenue and grants & 16.7 & 15.9 & 16.2 & 18.0 & 17.7 & 15.1 & 17.0 & 19.1 & 18.8 \\
\hline Total revenue & 16.4 & 15.1 & 15.6 & 17.4 & 15.6 & 14.2 & 16.1 & 18.0 & 18.3 \\
\hline Current revenue & 16.1 & 13.8 & 15.2 & 17.2 & 15.3 & 14.2 & 16.1 & 17.8 & 18.1 \\
\hline Tax revenue & 15.0 & 12.7 & 12.5 & 14.2 & 12.3 & 11.7 & 12.4 & 13.9 & 14.3 \\
\hline Income tax & 4.4 & 2.9 & 2.2 & 2.6 & 2.4 & 2.1 & 2.7 & 2.9 & 2.9 \\
\hline VAT & 4.4 & 5.3 & 5.6 & 5.8 & 4.6 & 4.6 & 5.7 & 6.4 & 6.6 \\
\hline Excises & 1.8 & 1.1 & 1.5 & 2.1 & 2.6 & 2.3 & 1.5 & 1.4 & 1.4 \\
\hline Customs & 0.8 & 0.9 & 0.8 & 1.1 & 0.6 & 0.4 & 0.4 & 0.6 & 0.5 \\
\hline Land tax & 0.5 & 0.5 & 0.9 & 0.9 & 0.4 & 0.3 & 0.3 & 0.4 & 0.4 \\
\hline Road tax and Emergency Fund & 1.3 & 1.0 & 1.0 & 1.2 & 1.2 & 1.6 & 1.3 & 1.4 & 1.4 \\
\hline Retail sales tax & 1.0 & 0.5 & 0.3 & 0.4 & 0.4 & 0.4 & 0.4 & 0.5 & 0.6 \\
\hline Other & 0.7 & 0.5 & 0.2 & 0.1 & 0.1 & 0.1 & 0.1 & 0.3 & 0.6 \\
\hline Nontax revenue & 1.1 & 1.1 & 2.6 & 2.9 & 3.1 & 2.4 & 3.6 & 3.9 & 3.8 \\
\hline Capital revenue & 0.3 & 1.3 & 0.4 & 0.3 & 0.3 & 0.0 & 0.1 & 0.2 & 0.2 \\
\hline Grants & 0.3 & 0.9 & 0.6 & 0.6 & 2.0 & 0.9 & 0.8 & 1.1 & 0.5 \\
\hline Total expenditure (excluding net lending) & 29.7 & 24.8 & 25.4 & 29.3 & 30.8 & 25.4 & 22.8 & 24.8 & 24.5 \\
\hline Current expenditure & 25.0 & 21.0 & 21.6 & 22.7 & 20.3 & 17.2 & 17.5 & 18.9 & 19.6 \\
\hline Wages and Social Fund contributions & 9.5 & 7.2 & 6.7 & 7.7 & 5.7 & 5.1 & 5.8 & 6.5 & 6.8 \\
\hline Transfers and subsidies & 6.2 & 4.2 & 3.1 & 3.0 & 2.2 & 2.5 & 3.0 & 3.4 & 3.2 \\
\hline Transfers to Social Fund & 1.2 & 1.7 & 1.8 & 1.5 & 1.0 & 0.6 & 0.5 & 1.1 & 1.3 \\
\hline Social benefits and pension supplements & 0.7 & 0.4 & 0.4 & 0.5 & 0.4 & 0.3 & 0.5 & 0.6 & 0.6 \\
\hline General transfer (subsidy) & 0.6 & 1.3 & 1.5 & 1.0 & 0.6 & 0.2 & 0.0 & 0.5 & 0.7 \\
\hline Interest & 0.4 & 1.2 & 1.7 & 2.1 & 2.9 & 2.3 & 1.7 & 1.6 & 1.5 \\
\hline Domestic & 0.1 & 0.3 & 0.8 & 0.9 & 0.5 & 0.5 & 0.4 & 0.3 & 0.4 \\
\hline Foreign & 0.4 & 0.9 & 0.9 & 1.2 & 2.5 & 1.8 & 1.3 & 1.3 & 1.1 \\
\hline Purchases of other goods and services & 7.6 & 6.6 & 8.3 & 8.4 & 8.5 & 6.7 & 6.5 & 6.3 & 6.7 \\
\hline Capital expenditure (including PIP) & 4.8 & 3.8 & 3.8 & 6.6 & 10.5 & 8.1 & 5.3 & 5.9 & 4.8 \\
\hline Domestically financed capital expenditure $2 /$ & 1.0 & 0.7 & 0.7 & 0.9 & 1.1 & 1.4 & 0.9 & 1.2 & 1.2 \\
\hline Foreign financed PIP & 3.8 & 3.2 & 3.1 & 5.7 & 9.4 & 6.8 & 4.4 & 4.7 & 3.6 \\
\hline Financial balance & -13.0 & -8.9 & -9.2 & -11.3 & -13.1 & -10.2 & -5.9 & -5.7 & -5.7 \\
\hline Net lending & 3.4 & 0.4 & -0.1 & -0.5 & -0.4 & -0.5 & -0.7 & -0.6 & -0.5 \\
\hline Accrual surplus (+) / deficit (-) & -16.4 & -9.3 & -9.1 & -10.8 & -12.7 & -9.7 & -5.2 & -5.1 & -5.2 \\
\hline Net accumulation of expenditure arrears & -0.8 & -0.2 & 0.0 & 1.3 & -0.7 & -0.3 & 0.2 & -0.3 & -0.1 \\
\hline Rescheduling of external interest arrears 1 / & 0.0 & 0.0 & 0.0 & 0.0 & 1.5 & 0.8 & 0.0 & 0.0 & 0.0 \\
\hline Cash surplus (+) / deficit (-) & -17.3 & -9.5 & -9.2 & -9.5 & -11.9 & -9.2 & -5.0 & -5.4 & -5.2 \\
\hline Total financing & 17.3 & 9.5 & 9.2 & 9.5 & 11.9 & 9.2 & 5.0 & 5.4 & 5.2 \\
\hline External financing & 9.1 & 6.9 & 8.0 & 8.9 & 14.5 & 8.8 & 5.7 & 5.2 & 4.2 \\
\hline Public investment program (PIP) & 3.8 & 3.2 & 3.1 & 5.7 & 9.4 & 6.8 & 4.4 & 4.8 & 3.4 \\
\hline Disbursements (BOP support) & 8.0 & 3.8 & 4.8 & 3.3 & 5.3 & 2.5 & 2.7 & 0.5 & 0.3 \\
\hline Turkish loan & 0.9 & 0.4 & 0.4 & 0.0 & 0.0 & 0.0 & 0.0 & 0.0 & 0.0 \\
\hline Total amortization & -3.6 & -0.8 & -0.5 & -0.7 & -0.3 & -2.5 & -2.5 & -1.2 & -1.8 \\
\hline Arrears and rescheduling & $\ldots$ & 0.3 & 0.3 & 0.7 & 0.2 & 2.1 & 1.1 & 1.1 & 2.3 \\
\hline Domestic financing & 8.2 & 2.1 & 1.1 & 0.3 & -2.8 & 0.1 & -1.1 & 0.0 & 0.7 \\
\hline NBKR & 7.8 & 1.9 & 0.4 & 0.2 & -2.1 & -0.1 & -1.3 & 0.0 & 0.5 \\
\hline Commercial banks & 0.4 & 0.1 & 0.4 & 0.1 & -0.5 & 0.1 & 0.2 & 0.1 & 0.2 \\
\hline Nonbank & 0.0 & 0.1 & 0.3 & 0.0 & -0.3 & 0.0 & 0.0 & 0.0 & 0.0 \\
\hline Exceptional Financing & 0.0 & 0.5 & 0.1 & 0.2 & 0.2 & 0.3 & 0.4 & 0.2 & 0.4 \\
\hline \multicolumn{10}{|l|}{ Memorandum items: } \\
\hline Primary balance & -16.8 & -8.3 & -7.5 & -7.4 & -9.0 & -6.9 & -3.3 & -3.8 & -3.7 \\
\hline Domestic balance 2/ & -13.1 & -5.5 & -5.2 & -2.5 & -0.1 & -0.7 & 0.7 & 0.6 & -0.5 \\
\hline Underlying balance 3 / & -13.4 & -6.0 & -5.1 & -2.4 & -1.8 & -1.5 & -0.6 & -1.2 & -1.7 \\
\hline Socail Expenditure 4/ & 16.9 & 13.4 & 12.5 & 11.7 & 9.8 & 8.2 & 8.7 & 11.1 & 11.0 \\
\hline Subsidies to public enterprises & 1.0 & 0.9 & 0.5 & 0.6 & 0.7 & 0.7 & 0.8 & 1.0 & 0.8 \\
\hline
\end{tabular}

Sources: Kyrgyz authorities and Fund staff estimates.

1/ In 2000, includes interest rescheduled for bilateral loans.

2/ Primary balance excluding foreign interest payments and foreign loan financed PIP.

3/ Primary balance excluding revenues from gold projects, grant, and foreign loan financed PIP.

4/ Includes health, education, social security, social insurance, and housing. 
Table 26. Kyrgyz Republic: State Government Revenues, 1995-2003

\begin{tabular}{|c|c|c|c|c|c|c|c|c|c|}
\hline & 1995 & 1996 & 1997 & 1998 & 1999 & 2000 & 2001 & 2002 & 2003 \\
\hline & \multicolumn{9}{|c|}{ (In millions of soms) } \\
\hline Total revenue and grants & 2,703 & 3,728 & 4,973 & 6,149 & 8,608 & 9,896 & 12,544 & 14,402 & 15,666 \\
\hline Total revenue & 2,648 & 3,527 & 4,778 & 5,953 & 7,612 & 9,287 & 11,922 & 13,569 & 15,248 \\
\hline Current revenues & 2,599 & 3,233 & 4,652 & 5,863 & 7,464 & 9,264 & 11,864 & 13,439 & 15,112 \\
\hline Tax revenue & 2,423 & 2,968 & 3,847 & 4,867 & 5,972 & 7,675 & 9,188 & 10,475 & 11,916 \\
\hline Tax on income & 713 & 669 & 685 & 892 & 1,151 & 1,379 & 2,008 & 2,199 & 2,381 \\
\hline Personal income tax & 284 & 288 & 318 & 405 & 546 & 754 & 961 & 1,083 & 1,208 \\
\hline Corporate profit tax & 415 & 365 & 350 & 452 & 568 & 573 & 994 & 968 & 913 \\
\hline Other income taxes & 15 & 16 & 17 & 34 & 37 & 53 & 54 & 148 & 260 \\
\hline VAT & 705 & 1,248 & 1,728 & 1,966 & 2,253 & 2,976 & 4,221 & 4,794 & 5,526 \\
\hline Domestic & $\ldots$ & 1,107 & 1,145 & 958 & 1,106 & 1,237 & 1,352 & 1,531 & 1,580 \\
\hline Import & $\ldots$ & 141 & 583 & 1,008 & 1,147 & 1,739 & 2,870 & 3,263 & 3,946 \\
\hline Retail sales $\operatorname{tax} 1 /$ & 168 & 114 & 85 & 151 & 187 & 232 & 305 & 410 & 471 \\
\hline Excises & 298 & 246 & 451 & 722 & 1,253 & 1,518 & 1,103 & 1,082 & 1,164 \\
\hline Domestic & 113 & 141 & 280 & 407 & 664 & 1,052 & 614 & 554 & 594 \\
\hline Import & 186 & 102 & 171 & 315 & 588 & 466 & 489 & 528 & 570 \\
\hline Road tax & 76 & 80 & 107 & 133 & 184 & 470 & 410 & 379 & 399 \\
\hline Emergency Fund contributions & 141 & 159 & 212 & 288 & 383 & 589 & 580 & 651 & 729 \\
\hline Mineral development & $\ldots$ & $\ldots$ & $\ldots$ & $\ldots$ & 19 & 24 & 25 & 191 & 427 \\
\hline Land tax & 73 & 122 & 278 & 291 & 199 & 179 & 209 & 318 & 312 \\
\hline Miscellaneous local taxes & 0 & 0 & 0 & 0 & 16 & 18 & 21 & 28 & 38 \\
\hline Tax on property (excluding land tax) & 0 & 0 & 0 & 0 & 0 & 0 & 0 & 0 & 45 \\
\hline Taxes on international trade & 134 & 206 & 245 & 381 & 307 & 275 & 301 & 419 & 423 \\
\hline Other taxes & 114 & 125 & 56 & 43 & 23 & 14 & 4 & 3 & 1 \\
\hline Non-Tax Revenue & 176 & 265 & 805 & 997 & 1,491 & 1,588 & 2,677 & 2,964 & 3,195 \\
\hline Capital revenue & 49 & 293 & 126 & 90 & 148 & 24 & 57 & 130 & 137 \\
\hline \multirow[t]{2}{*}{ Grants } & 55 & 201 & 195 & 197 & 996 & 608 & 622 & 833 & 418 \\
\hline & \multicolumn{9}{|c|}{ (In percent of GDP) } \\
\hline Total revenue and grants & 16.7 & 15.9 & 16.2 & 18.0 & 17.7 & 15.1 & 17.0 & 19.1 & 18.8 \\
\hline Total revenue & 16.4 & 15.1 & 15.6 & 17.4 & 15.6 & 14.2 & 16.1 & 18.0 & 18.3 \\
\hline Current revenues & 16.1 & 13.8 & 15.2 & 17.2 & 15.3 & 14.2 & 16.1 & 17.8 & 18.1 \\
\hline Tax revenue & 15.0 & 12.7 & 12.5 & 14.2 & 12.3 & 11.7 & 12.4 & 13.9 & 14.3 \\
\hline Tax on income & 4.4 & 2.9 & 2.2 & 2.6 & 2.4 & 2.1 & 2.7 & 2.9 & 2.9 \\
\hline VAT & 4.4 & 5.3 & 5.6 & 5.8 & 4.6 & 4.6 & 5.7 & 6.4 & 6.6 \\
\hline Retail sales tax 1/ & 1.0 & 0.5 & 0.3 & 0.4 & 0.4 & 0.4 & 0.4 & 0.5 & 0.6 \\
\hline Excises & 1.8 & 1.1 & 1.5 & 2.1 & 2.6 & 2.3 & 1.5 & 1.4 & 1.4 \\
\hline Road tax & 0.5 & 0.3 & 0.3 & 0.4 & 0.4 & 0.7 & 0.6 & 0.5 & 0.5 \\
\hline Emergency Fund contributions & 0.9 & 0.7 & 0.7 & 0.8 & 0.8 & 0.9 & 0.8 & 0.9 & 0.9 \\
\hline Mineral development & $\ldots$ & $\ldots$ & $\ldots$ & $\ldots$ & 0.0 & 0.0 & 0.0 & 0.3 & 0.5 \\
\hline Land tax & 0.5 & 0.5 & 0.9 & 0.9 & 0.4 & 0.3 & 0.3 & 0.4 & 0.4 \\
\hline Miscellaneous local taxes & 0.0 & 0.0 & 0.0 & 0.0 & 0.0 & 0.0 & 0.0 & 0.0 & 0.0 \\
\hline Tax on property (excluding land tax) & 0.0 & 0.0 & 0.0 & 0.0 & 0.0 & 0.0 & 0.0 & 0.0 & 0.1 \\
\hline Taxes on international trade & 0.8 & 0.9 & 0.8 & 1.1 & 0.6 & 0.4 & 0.4 & 0.6 & 0.5 \\
\hline Other taxes & 0.7 & 0.5 & 0.2 & 0.1 & 0.0 & 0.0 & 0.0 & 0.0 & 0.0 \\
\hline Non-Tax Revenue & 1.1 & 1.1 & 2.6 & 2.9 & 3.1 & 2.4 & 3.6 & 3.9 & 3.8 \\
\hline Capital revenue & 0.3 & 1.3 & 0.4 & 0.3 & 0.3 & 0.0 & 0.1 & 0.2 & 0.2 \\
\hline \multirow{2}{*}{ Grants } & 0.3 & 0.9 & 0.6 & 0.6 & 2.0 & 0.9 & 0.8 & 1.1 & 0.5 \\
\hline & \multicolumn{9}{|c|}{ (In percent of total) } \\
\hline Total revenue and grants & 100.0 & 100.0 & 100.0 & 100.0 & 100.0 & 100.0 & 100.0 & 100.0 & 100.0 \\
\hline Total revenue & 98.0 & 94.6 & 96.1 & 96.8 & 88.4 & 93.9 & 95.0 & 94.2 & 97.3 \\
\hline Current revenues & 96.2 & 86.7 & 93.5 & 95.3 & 86.7 & 93.6 & 94.6 & 93.3 & 96.5 \\
\hline Tax revenue & 89.7 & 79.6 & 77.4 & 79.1 & 69.4 & 77.6 & 73.2 & 72.7 & 76.1 \\
\hline Tax on income & 26.4 & 17.9 & 13.8 & 14.5 & 13.4 & 13.9 & 16.0 & 15.3 & 15.2 \\
\hline VAT & 26.1 & 33.5 & 34.8 & 32.0 & 26.2 & 30.1 & 33.7 & 33.3 & 35.3 \\
\hline Retail sales tax 1/ & 6.2 & 3.1 & 1.7 & 2.5 & 2.2 & 2.3 & 2.4 & 2.8 & 3.0 \\
\hline Excises & 11.0 & 6.6 & 9.1 & 11.7 & 14.6 & 15.3 & 8.8 & 7.5 & 7.4 \\
\hline Road tax & 2.8 & 2.1 & 2.1 & 2.2 & 2.1 & 4.7 & 3.3 & 2.6 & 2.5 \\
\hline Emergency Fund contributions & 5.2 & 4.3 & 4.3 & 4.7 & 4.4 & 6.0 & 4.6 & 4.5 & 4.7 \\
\hline Mineral development & $\ldots$ & $\ldots$ & $\ldots$ & $\ldots$ & 0.2 & 0.2 & 0.2 & 1.3 & 2.7 \\
\hline Land tax & 2.7 & 3.3 & 5.6 & 4.7 & 2.3 & 1.8 & 1.7 & 2.2 & 2.0 \\
\hline Miscellaneous local taxes & 0.0 & 0.0 & 0.0 & 0.0 & 0.2 & 0.2 & 0.2 & 0.2 & 0.2 \\
\hline Tax on property (excluding land tax) & 0.0 & 0.0 & 0.0 & 0.0 & 0.0 & 0.0 & 0.0 & 0.0 & 0.3 \\
\hline Taxes on international trade & 5.0 & 5.5 & 4.9 & 6.2 & 3.6 & 2.8 & 2.4 & 2.9 & 2.7 \\
\hline Other taxes & 4.2 & 3.3 & 1.1 & 0.7 & 0.3 & 0.1 & 0.0 & 0.0 & 0.0 \\
\hline Non-Tax Revenue & 6.5 & 7.1 & 16.2 & 16.2 & 17.3 & 16.1 & 21.3 & 20.6 & 20.4 \\
\hline Capital revenue & 1.8 & 7.9 & 2.5 & 1.5 & 1.7 & 0.2 & 0.5 & 0.9 & 0.9 \\
\hline Grants & 2.0 & 5.4 & 3.9 & 3.2 & 11.6 & 6.1 & 5.0 & 5.8 & 2.7 \\
\hline
\end{tabular}

Sources: Kyrgyz authorities and Fund staff estimates.

1/ Local tax since 1997 
Table 27. Kyrgyz Republic: State Government Expenditure by Functional Classification, 1995-2003

\begin{tabular}{|c|c|c|c|c|c|c|c|c|c|}
\hline & 1995 & 1996 & 1997 & 1998 & 1999 & 2000 & 2001 & 2002 & 2003 \\
\hline & \multicolumn{9}{|c|}{ (In millions of soms) } \\
\hline Total expenditure including net lending & 5,358 & 5,905 & 7,778 & 9,843 & 14,802 & 16,261 & 16,371 & 18,267 & 19,989 \\
\hline Total cash expenditure (excl. net lending and PIP) & 4,325 & 5,121 & 6,852 & 7,616 & 10,763 & 12,335 & 13,487 & 15,400 & 17,420 \\
\hline General public services & 362 & 580 & 850 & 1,015 & 1,375 & 1,756 & 2,105 & 2,255 & 2,644 \\
\hline Defense & 237 & 314 & 482 & 491 & 844 & 1,137 & 981 & 1,098 & 1,287 \\
\hline Public order and safety affairs & 334 & 385 & 473 & 421 & 465 & 744 & 752 & 957 & 1,121 \\
\hline Education & 1,051 & 1,223 & 1,514 & 1,682 & 1,991 & 2,293 & 2,848 & 3,350 & 3,753 \\
\hline Health & 590 & 733 & 977 & 962 & 1,125 & 1,296 & 1,379 & 1,527 & 1,630 \\
\hline Social security and welfare affairs & 899 & 885 & 1,055 & 981 & 1,198 & 1,114 & 1,417 & 2,341 & 2,588 \\
\hline Social insurance & 796 & 793 & 878 & 758 & 495 & 372 & 398 & 840 & 1,144 \\
\hline Social security & 104 & 92 & 177 & 224 & 704 & 742 & 1,019 & 1,501 & 1,444 \\
\hline Housing and community services & 183 & 298 & 280 & 374 & 465 & 667 & 801 & 1,131 & 1,193 \\
\hline Recreational, cultural and religious activities & 127 & 125 & 148 & 187 & 308 & 337 & 295 & 393 & 484 \\
\hline Energy complex (electricity production) & 1 & 0 & 8 & 146 & 77 & 23 & 244 & 221 & 163 \\
\hline Agriculture, water resources, forestry & 171 & 181 & 238 & 350 & 389 & 476 & 607 & 744 & 784 \\
\hline Mining and mineral resources & 61 & 44 & 67 & 68 & 81 & 330 & 103 & 221 & 283 \\
\hline Transportation and communication & 70 & 99 & 170 & 228 & 523 & 465 & 420 & 668 & 565 \\
\hline Other economic affairs and services & 50 & 100 & 203 & 53 & 39 & 48 & 49 & 41 & 46 \\
\hline Interest $1 /$ & 68 & 284 & 520 & 715 & 1,436 & 1,507 & 1,228 & 1,238 & 1,280 \\
\hline Unclassified expenditures 2/ & 120 & -130 & -133 & -58 & 447 & 141 & 259 & -786 & -401 \\
\hline Net lending & 556 & 101 & -17 & -165 & -189 & -311 & -509 & -443 & -414 \\
\hline Net accumulation of arrears & -133 & -56 & -7 & 441 & -351 & -175 & 155 & -231 & -53 \\
\hline Foreign financed PIP & 610 & 739 & 949 & 1,952 & 4,578 & 4,412 & 3,239 & 3,541 & 3,036 \\
\hline \multirow[t]{2}{*}{ Memorandum item: interest paid } & $\ldots$ & $\ldots$ & $\ldots$ & $\ldots$ & 672 & 914 & 1,045 & 674 & 678 \\
\hline & \multicolumn{9}{|c|}{ (In percent of GDP) } \\
\hline Total cash expenditure (excl. net lending and PIP) & 26.8 & 21.9 & 22.3 & 22.3 & 22.1 & 18.9 & 18.3 & 20.4 & 20.9 \\
\hline General public services & 2.2 & 2.5 & 2.8 & 3.0 & 2.8 & 2.7 & 2.8 & 3.0 & 3.2 \\
\hline Defense & 1.5 & 1.3 & 1.6 & 1.4 & 1.7 & 1.7 & 1.3 & 1.5 & 1.5 \\
\hline Public order and safety affairs & 2.1 & 1.6 & 1.5 & 1.2 & 1.0 & 1.1 & 1.0 & 1.3 & 1.3 \\
\hline Education & 6.5 & 5.2 & 4.9 & 4.9 & 4.1 & 3.5 & 3.9 & 4.4 & 4.5 \\
\hline Health & 3.7 & 3.1 & 3.2 & 2.8 & 2.3 & 2.0 & 1.9 & 2.0 & 2.0 \\
\hline Social security and welfare affairs & 5.6 & 3.8 & 3.4 & 2.9 & 2.5 & 1.7 & 1.9 & 3.1 & 3.1 \\
\hline Social insurance & 4.9 & 3.4 & 2.9 & 2.2 & 1.0 & 0.6 & 0.5 & 1.1 & 1.4 \\
\hline Social security & 0.6 & 0.4 & 0.6 & 0.7 & 1.4 & 1.1 & 1.4 & 2.0 & 1.7 \\
\hline Housing and community services & 1.1 & 1.3 & 0.9 & 1.1 & 1.0 & 1.0 & 1.1 & 1.5 & 1.4 \\
\hline Recreational, cultural and religious activities & 0.8 & 0.5 & 0.5 & 0.5 & 0.6 & 0.5 & 0.4 & 0.5 & 0.6 \\
\hline Energy complex (electricity production) & 0.0 & 0.0 & 0.0 & 0.4 & 0.2 & 0.0 & 0.3 & 0.3 & 0.2 \\
\hline Agriculture, water resources, forestry & 1.1 & 0.8 & 0.8 & 1.0 & 0.8 & 0.7 & 0.8 & 1.0 & 0.9 \\
\hline Mining and mineral resources & 0.4 & 0.2 & 0.2 & 0.2 & 0.2 & 0.5 & 0.1 & 0.3 & 0.3 \\
\hline Transportation and communication & 0.4 & 0.4 & 0.6 & 0.7 & 1.1 & 0.7 & 0.6 & 0.9 & 0.7 \\
\hline Other economic affairs and services & 0.3 & 0.4 & 0.7 & 0.2 & 0.1 & 0.1 & 0.1 & 0.1 & 0.1 \\
\hline Interest $1 /$ & 0.4 & 1.2 & 1.7 & 2.1 & 2.9 & 2.3 & 1.7 & 1.6 & 1.5 \\
\hline \multirow[t]{2}{*}{ Unclassified expenditures 2/ } & 0.7 & -0.6 & -0.4 & -0.2 & 0.9 & 0.2 & 0.4 & -1.0 & -0.5 \\
\hline & \multicolumn{9}{|c|}{ (In percent of total) } \\
\hline Total cash expenditure (excl. net lending and PIP) & 100.0 & 100.0 & 100.0 & 100.0 & 100.0 & 100.0 & 100.0 & 100.0 & 100.0 \\
\hline General public services & 8.4 & 11.3 & 12.4 & 13.3 & 12.8 & 14.2 & 15.6 & 14.6 & 15.2 \\
\hline Defense & 5.5 & 6.1 & 7.0 & 6.4 & 7.8 & 9.2 & 7.3 & 7.1 & 7.4 \\
\hline Public order and safety affairs & 7.7 & 7.5 & 6.9 & 5.5 & 4.3 & 6.0 & 5.6 & 6.2 & 6.4 \\
\hline Education & 24.3 & 23.9 & 22.1 & 22.1 & 18.5 & 18.6 & 21.1 & 21.8 & 21.5 \\
\hline Health & 13.7 & 14.3 & 14.3 & 12.6 & 10.5 & 10.5 & 10.2 & 9.9 & 9.4 \\
\hline Social security and welfare affairs & 20.8 & 17.3 & 15.4 & 12.9 & 11.1 & 9.0 & 10.5 & 15.2 & 14.9 \\
\hline Social insurance & 18.4 & 15.5 & 12.8 & 9.9 & 4.6 & 3.0 & 3.0 & 5.5 & 6.6 \\
\hline Social security & 2.4 & 1.8 & 2.6 & 2.9 & 6.5 & 6.0 & 7.6 & 9.7 & 8.3 \\
\hline Housing and community services & 4.2 & 5.8 & 4.1 & 4.9 & 4.3 & 5.4 & 5.9 & 7.3 & 6.9 \\
\hline Recreational, cultural and religious activities & 2.9 & 2.4 & 2.2 & 2.5 & 2.9 & 2.7 & 2.2 & 2.6 & 2.8 \\
\hline Energy complex (electricity production) & 0.0 & 0.0 & 0.1 & 1.9 & 0.7 & 0.2 & 1.8 & 1.4 & 0.9 \\
\hline Agriculture, water resources, forestry & 4.0 & 3.5 & 3.5 & 4.6 & 3.6 & 3.9 & 4.5 & 4.8 & 4.5 \\
\hline Mining and mineral resources & 1.4 & 0.9 & 1.0 & 0.9 & 0.8 & 2.7 & 0.8 & 1.4 & 1.6 \\
\hline Transportation and communication & 1.6 & 1.9 & 2.5 & 3.0 & 4.9 & 3.8 & 3.1 & 4.3 & 3.2 \\
\hline Other economic affairs and services & 1.1 & 2.0 & 3.0 & 0.7 & 0.4 & 0.4 & 0.4 & 0.3 & 0.3 \\
\hline Interest $1 /$ & 1.6 & 5.6 & 7.6 & 9.4 & 13.3 & 12.2 & 9.1 & 8.0 & 7.3 \\
\hline Unclassified expenditures $2 /$ & 2.8 & -2.5 & -1.9 & -0.8 & 4.2 & 1.1 & 1.9 & -5.1 & -2.3 \\
\hline
\end{tabular}

Sources: Kyrgyz authorities and Fund staff estimates.

1/ Accrual basis.

2/ Mainly net carry-forward expenditures, or "final settlements". 
Table 28. Kyrgyz Republic: Social Fund Financial Accounts, 1995-2003

\begin{tabular}{|c|c|c|c|c|c|c|c|c|c|}
\hline & 1995 & 1996 & 1997 & 1998 & 1999 & 2000 & 2001 & 2002 & 2003 \\
\hline & \multicolumn{9}{|c|}{ (In millions of soms) } \\
\hline Total revenues & 1,112 & 1,472 & 1,770 & 1,981 & 2,446 & 2,909 & 3,306 & 3,573 & 3,897 \\
\hline Total contribution (payroll tax) collection & 1,112 & 1,472 & 1,770 & 1,981 & 2,440 & 2,877 & 3,218 & 3,516 & 3,840 \\
\hline Pension Fund & 815 & 1,016 & 1,312 & 1,299 & 1,480 & 1,967 & 2,351 & 2,493 & 2,994 \\
\hline Social Insurance Fund & 155 & 202 & 170 & 159 & 186 & 159 & 155 & 154 & 102 \\
\hline Employment Fund & 142 & 66 & 86 & 101 & 133 & 147 & 132 & 109 & 102 \\
\hline Medical Insurance Fund & \multirow[t]{2}{*}{$\cdots$} & $\ldots$ & 41 & 115 & 201 & 138 & 166 & 185 & 199 \\
\hline Pension Fund arrear collections & & 187 & 161 & 307 & 440 & 465 & 414 & 575 & 444 \\
\hline Other revenues and bank credits & $\ldots$ & $\ldots$ & $\ldots$ & 0 & 6 & 32 & 88 & 57 & 57 \\
\hline Expenditure & 1,360 & 1,959 & 2,321 & 2,822 & 2,958 & 3,410 & 3,945 & 4,510 & 4,867 \\
\hline Pension Fund 1/ & 1,196 & 1,714 & 2,092 & 2,409 & 2,632 & 2,978 & 3,504 & 4,070 & 4,457 \\
\hline Social Insurance Fund 1/ & 105 & 106 & 138 & 129 & 123 & 128 & 143 & 150 & 147 \\
\hline Employment Fund 1/ & 35 & 59 & 74 & 88 & 73 & 122 & 97 & 106 & 107 \\
\hline Medical Fund 1/ & 0 & 0 & 9 & 31 & 73 & 89 & 81 & 142 & 280 \\
\hline Net accumulation of arrears ('-' repayment) & 24 & 79 & 8 & 165 & 57 & 93 & 120 & 43 & -123 \\
\hline Financial balance & -248 & -487 & -551 & -841 & -512 & -501 & -640 & -937 & -971 \\
\hline Financing & 248 & 487 & 551 & 841 & 512 & 501 & 640 & 937 & 971 \\
\hline Transfers to Social Fund & 202 & 397 & 564 & 504 & 489 & 372 & 398 & 840 & 1,122 \\
\hline Social benefits and pension supplements & 111 & 104 & 118 & 160 & 189 & 222 & 398 & 435 & 534 \\
\hline General transfer (subsidy) & 91 & 293 & 446 & 344 & 300 & 150 & 0 & 405 & 588 \\
\hline Net accumulation of arrears & 24 & 79 & 8 & 165 & 57 & 93 & 120 & 43 & -123 \\
\hline \multirow[t]{2}{*}{ Change in cash balance ('-' accumulation) } & 23 & 10 & -21 & 172 & -34 & 36 & 121 & 54 & -28 \\
\hline & \multicolumn{9}{|c|}{ (In percent of GDP) } \\
\hline Total revenues & 6.9 & 6.3 & 5.8 & 5.8 & 5.0 & 4.5 & 4.5 & 4.7 & 4.7 \\
\hline Total contribution (payroll tax) collection & 6.9 & 6.3 & 5.8 & 5.8 & 5.0 & 4.4 & 4.4 & 4.7 & 4.6 \\
\hline Pension Fund & 5.0 & 4.3 & 4.3 & 3.8 & 3.0 & 3.0 & 3.2 & 3.3 & 3.6 \\
\hline Social Insurance Fund & 1.0 & 0.9 & 0.6 & 0.5 & 0.4 & 0.2 & 0.2 & 0.2 & 0.1 \\
\hline Employment Fund & 0.9 & 0.3 & 0.3 & 0.3 & 0.3 & 0.2 & 0.2 & 0.1 & 0.1 \\
\hline Medical Insurance Fund & $\ldots$ & $\ldots$ & 0.1 & 0.3 & 0.4 & 0.2 & 0.2 & 0.2 & 0.2 \\
\hline Pension Fund arrear collections & $\ldots$ & 0.8 & 0.5 & 0.9 & 0.9 & 0.7 & 0.6 & 0.8 & 0.5 \\
\hline Other revenues and bank credits & $\ldots$ & $\ldots$ & $\ldots$ & 0.0 & 0.0 & 0.0 & 0.1 & 0.1 & 0.1 \\
\hline Expenditure 1/ & 8.4 & 8.4 & 7.6 & 8.3 & 6.1 & 5.2 & 5.3 & 6.0 & 5.8 \\
\hline Pension Fund & 7.4 & 7.3 & 6.8 & 7.0 & 5.4 & 4.6 & 4.7 & 5.4 & 5.3 \\
\hline Social Insurance Fund & 0.7 & 0.5 & 0.4 & 0.4 & 0.3 & 0.2 & 0.2 & 0.2 & 0.2 \\
\hline Employment Fund & 0.2 & 0.3 & 0.2 & 0.3 & 0.2 & 0.2 & 0.1 & 0.1 & 0.1 \\
\hline Medical Fund & 0.0 & 0.0 & 0.0 & 0.1 & 0.1 & 0.1 & 0.1 & 0.2 & 0.3 \\
\hline Financial balance & -1.5 & -2.1 & -1.8 & -2.5 & -1.1 & -0.8 & -0.9 & -1.2 & -1.2 \\
\hline Financing & 1.5 & 2.1 & 1.8 & 2.5 & 1.1 & 0.8 & 0.9 & 1.2 & 1.2 \\
\hline Transfers to Social Fund & 1.2 & 1.7 & 1.8 & 1.5 & 1.0 & 0.6 & 0.5 & 1.1 & 1.3 \\
\hline Social benefits and pension supplements & 0.7 & 0.4 & 0.4 & 0.5 & 0.4 & 0.3 & 0.5 & 0.6 & 0.6 \\
\hline General transfer (subsidy) & 0.6 & 1.3 & 1.5 & 1.0 & 0.6 & 0.2 & 0.0 & 0.5 & 0.7 \\
\hline Net accumulation of arrears & 0.1 & 0.3 & 0.0 & 0.5 & 0.1 & 0.1 & 0.2 & 0.1 & -0.1 \\
\hline Change in cash balance ('-' accumulation) & 0.1 & 0.0 & -0.1 & 0.5 & -0.1 & 0.1 & 0.2 & 0.1 & 0.0 \\
\hline
\end{tabular}

Source: Kyrgyz authorities and Fund staff estimates.

1/ On a cash basis. 


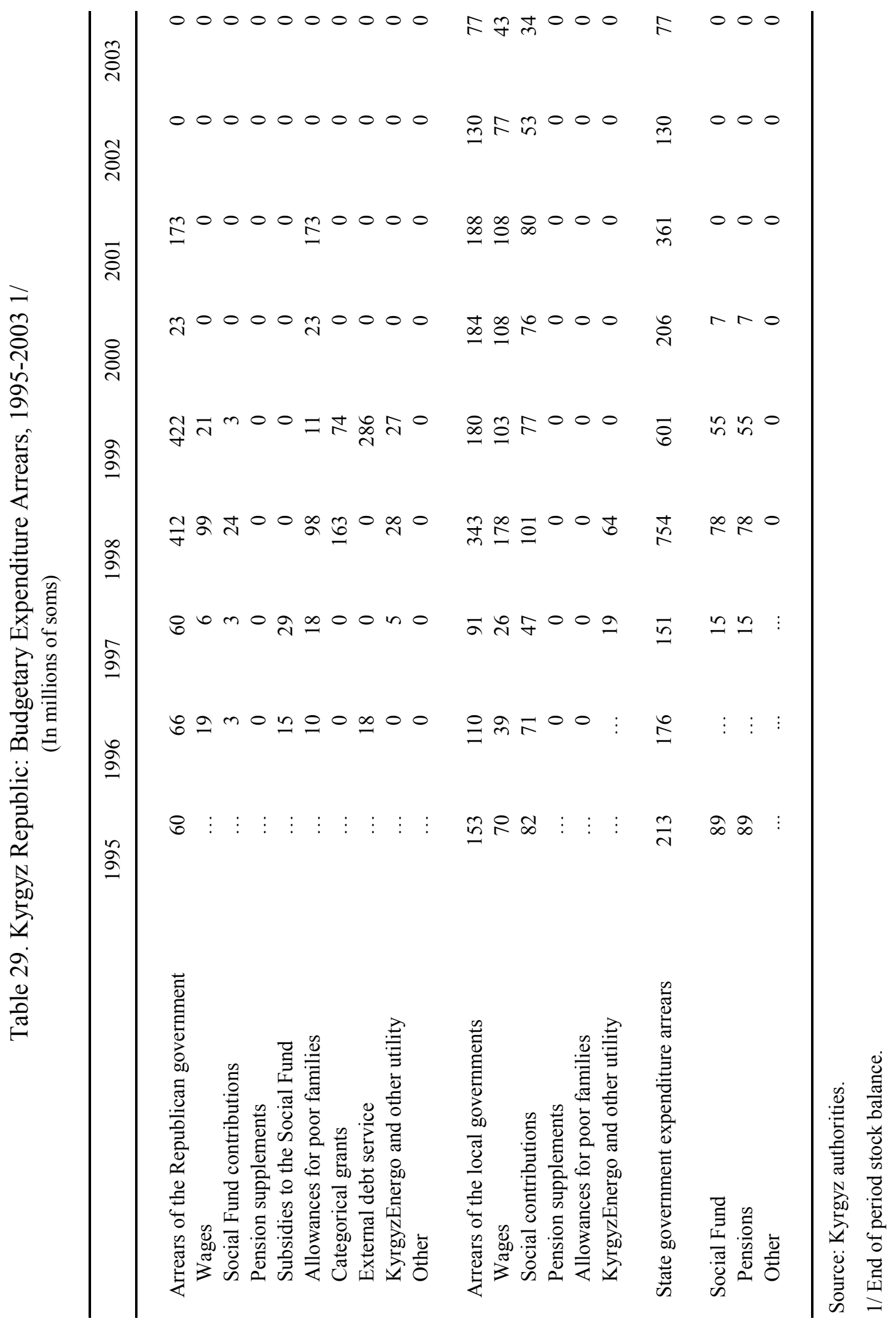


Table 30. Kyrgyz Republic: State Government Wage Bill by Sector, 1999-2003

\begin{tabular}{|c|c|c|c|c|c|c|c|c|c|c|c|}
\hline & & 1999 & 2000 & 2001 & 2002 & 2003 & 1999 & 2000 & 2001 & 2002 & 2003 \\
\hline & & \multicolumn{5}{|c|}{ (In millions of soms) } & \multicolumn{5}{|c|}{ (In percent of total) } \\
\hline \multicolumn{2}{|c|}{ Total wage and social fund contribution } & 2,986 & 3,501 & 4,296 & 4,934 & 5,705 & 100.0 & 100.0 & 100.0 & 100.0 & 100.0 \\
\hline I & General public services & 576 & 702 & 1,006 & 1,108 & 1,239 & 19.3 & 20.0 & 23.4 & 22.5 & 21.7 \\
\hline II & Defense & 122 & 341 & 390 & 449 & 512 & 4.1 & 9.8 & 9.1 & 9.1 & 9.0 \\
\hline III & Public order & 189 & 261 & 324 & 412 & 566 & 6.3 & 7.5 & 7.6 & 8.3 & 9.9 \\
\hline IV & Education & 1,216 & 1,280 & 1,497 & 1,689 & 1,968 & 40.7 & 36.6 & 34.8 & 34.2 & 34.5 \\
\hline $\mathrm{V}$ & Health & 582 & 585 & 654 & 719 & 813 & 19.5 & 16.7 & 15.2 & 14.6 & 14.3 \\
\hline VI & Social security and welfare affairs & 40 & 50 & 74 & 90 & 98 & 1.3 & 1.4 & 1.7 & 1.8 & 1.7 \\
\hline VII & Housing/comm. service & 4 & 4 & 6 & 7 & 11 & 0.1 & 0.1 & 0.1 & 0.1 & 0.2 \\
\hline VIII & Recreation/culture & 84 & 85 & 104 & 125 & 150 & 2.8 & 2.4 & 2.4 & 2.5 & 2.6 \\
\hline IX & Fuel and energy budget & 1 & 1 & 1 & 1 & 2 & 0.0 & 0.0 & 0.0 & 0.0 & 0.0 \\
\hline $\mathrm{X}$ & Agriculture & 123 & 136 & 172 & 206 & 229 & 4.1 & 3.9 & 4.0 & 4.2 & 4.0 \\
\hline $\mathrm{XI}$ & Mining & 43 & 47 & 51 & 52 & 35 & 1.4 & 1.3 & 1.2 & 1.0 & 0.6 \\
\hline XII & Transport and communications & 1 & 1 & 7 & 61 & 65 & 0.0 & 0.0 & 0.2 & 1.2 & 1.1 \\
\hline XIII & Other economic affairs and services & 6 & 6 & 8 & 15 & 16 & 0.2 & 0.2 & 0.2 & 0.3 & 0.3 \\
\hline XIV & Other & 0 & 0 & 0 & 0 & 0 & 0.0 & 0.0 & 0.0 & 0.0 & 0.0 \\
\hline \multicolumn{2}{|c|}{ Central government (Republican budget) } & 1,284 & 1,658 & 2,181 & 2,519 & 2,925 & 43.0 & 47.4 & 50.8 & 51.1 & 51.3 \\
\hline I & General public services & 462 & 540 & 800 & 850 & 940 & 15.5 & 15.4 & 18.6 & 17.2 & 16.5 \\
\hline II & Defense & 120 & 339 & 387 & 445 & 508 & 4.0 & 9.7 & 9.0 & 9.0 & 8.9 \\
\hline III & Public order & 179 & 246 & 308 & 395 & 544 & 6.0 & 7.0 & 7.2 & 8.0 & 9.5 \\
\hline IV & Education & 220 & 213 & 262 & 282 & 334 & 7.4 & 6.1 & 6.1 & 5.7 & 5.9 \\
\hline $\mathrm{V}$ & Health & 101 & 105 & 125 & 132 & 153 & 3.4 & 3.0 & 2.9 & 2.7 & 2.7 \\
\hline VI & Social security and welfare affairs & 16 & 18 & 29 & 38 & 42 & 0.5 & 0.5 & 0.7 & 0.8 & 0.7 \\
\hline VII & Housing/comm. service & 1 & 1 & 1 & 1 & 1 & 0.0 & 0.0 & 0.0 & 0.0 & 0.0 \\
\hline VIII & Recreation/culture & 39 & 36 & 50 & 58 & 73 & 1.3 & 1.0 & 1.2 & 1.2 & 1.3 \\
\hline IX & Fuel and energy budget & 1 & 1 & 1 & 1 & 2 & 0.0 & 0.0 & 0.0 & 0.0 & 0.0 \\
\hline $\mathrm{X}$ & Agriculture & 96 & 105 & 153 & 191 & 212 & 3.2 & 3.0 & 3.6 & 3.9 & 3.7 \\
\hline XI & Mining & 43 & 46 & 50 & 51 & 34 & 1.4 & 1.3 & 1.2 & 1.0 & 0.6 \\
\hline XII & Transport and communications & 1 & 1 & 7 & 61 & 65 & 0.0 & 0.0 & 0.2 & 1.2 & 1.1 \\
\hline XIII & Other economic affairs and services & 6 & 6 & 8 & 15 & 16 & 0.2 & 0.2 & 0.2 & 0.3 & 0.3 \\
\hline XIV & Other & 0 & 0 & 0 & 0 & 0 & 0.0 & 0.0 & 0.0 & 0.0 & 0.0 \\
\hline \multicolumn{2}{|c|}{ Local governments (local budgets) } & 1,702 & 1,843 & 2,115 & 2,415 & 2,780 & 57.0 & 52.6 & 49.2 & 48.9 & 48.7 \\
\hline $\mathrm{I}$ & General public services & 114 & 161 & 205 & 258 & 298 & 3.8 & 4.6 & 4.8 & 5.2 & 5.2 \\
\hline II & Defense & 2 & 2 & 3 & 4 & 4 & 0.1 & 0.1 & 0.1 & 0.1 & 0.1 \\
\hline III & Public order & 10 & 15 & 17 & 17 & 23 & 0.3 & 0.4 & 0.4 & 0.3 & 0.4 \\
\hline IV & Education & 997 & 1,068 & 1,235 & 1,406 & 1,634 & 33.4 & 30.5 & 28.7 & 28.5 & 28.6 \\
\hline $\mathrm{V}$ & Health & 481 & 480 & 530 & 586 & 660 & 16.1 & 13.7 & 12.3 & 2.7 & 11.6 \\
\hline VI & Social security and welfare affairs & 23 & 32 & 45 & 52 & 56 & 0.8 & 0.9 & 1.0 & 1.1 & 1.0 \\
\hline VII & Housing/comm. service & 3 & 4 & 6 & 6 & 10 & 0.1 & 0.1 & 0.1 & 0.1 & 0.2 \\
\hline VIII & Recreation/culture & 44 & 49 & 55 & 67 & 78 & 1.5 & 1.4 & 1.3 & 1.4 & 1.4 \\
\hline IX & Fuel and energy budget & 0 & 0 & 0 & 0 & 0 & 0.0 & 0.0 & 0.0 & 0.0 & 0.0 \\
\hline$X$ & Agriculture & 27 & 31 & 19 & 15 & 17 & 0.9 & 0.9 & 0.4 & 0.3 & 0.3 \\
\hline $\mathrm{XI}$ & Mining & 0 & 1 & 1 & 1 & 1 & 0.0 & 0.0 & 0.0 & 0.0 & 0.0 \\
\hline XII & Transport and communications & 0 & 0 & 0 & 0 & 0 & 0.0 & 0.0 & 0.0 & 0.0 & 0.0 \\
\hline XIII & Other economic affairs and services & 0 & 0 & 0 & 0 & 0 & 0.0 & 0.0 & 0.0 & 0.0 & 0.0 \\
\hline XIV & Other & 0 & 0 & 0 & 0 & 0 & 0.0 & 0.0 & 0.0 & 0.0 & 0.0 \\
\hline
\end{tabular}

Sources: Kyrgyz authorities. 


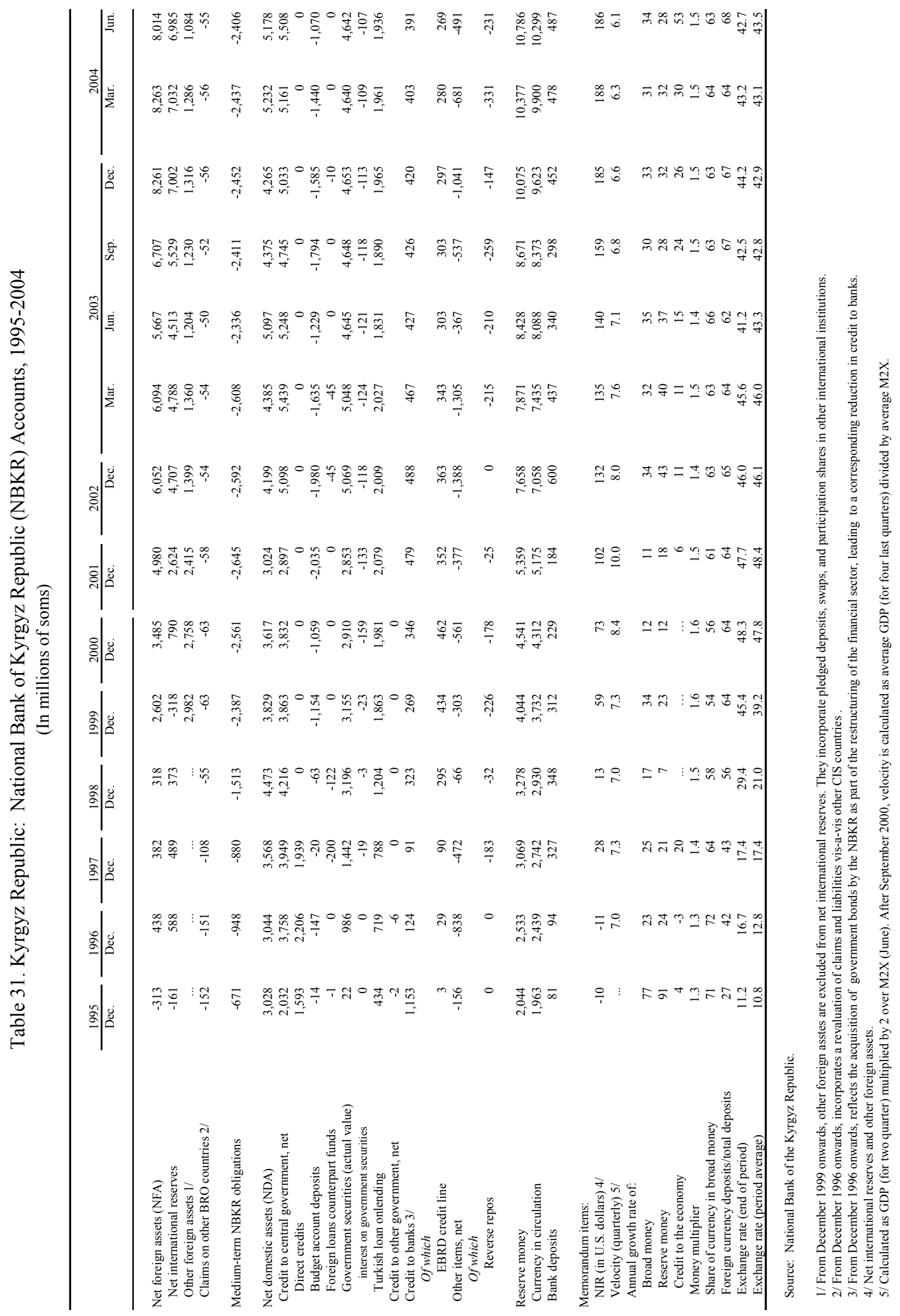




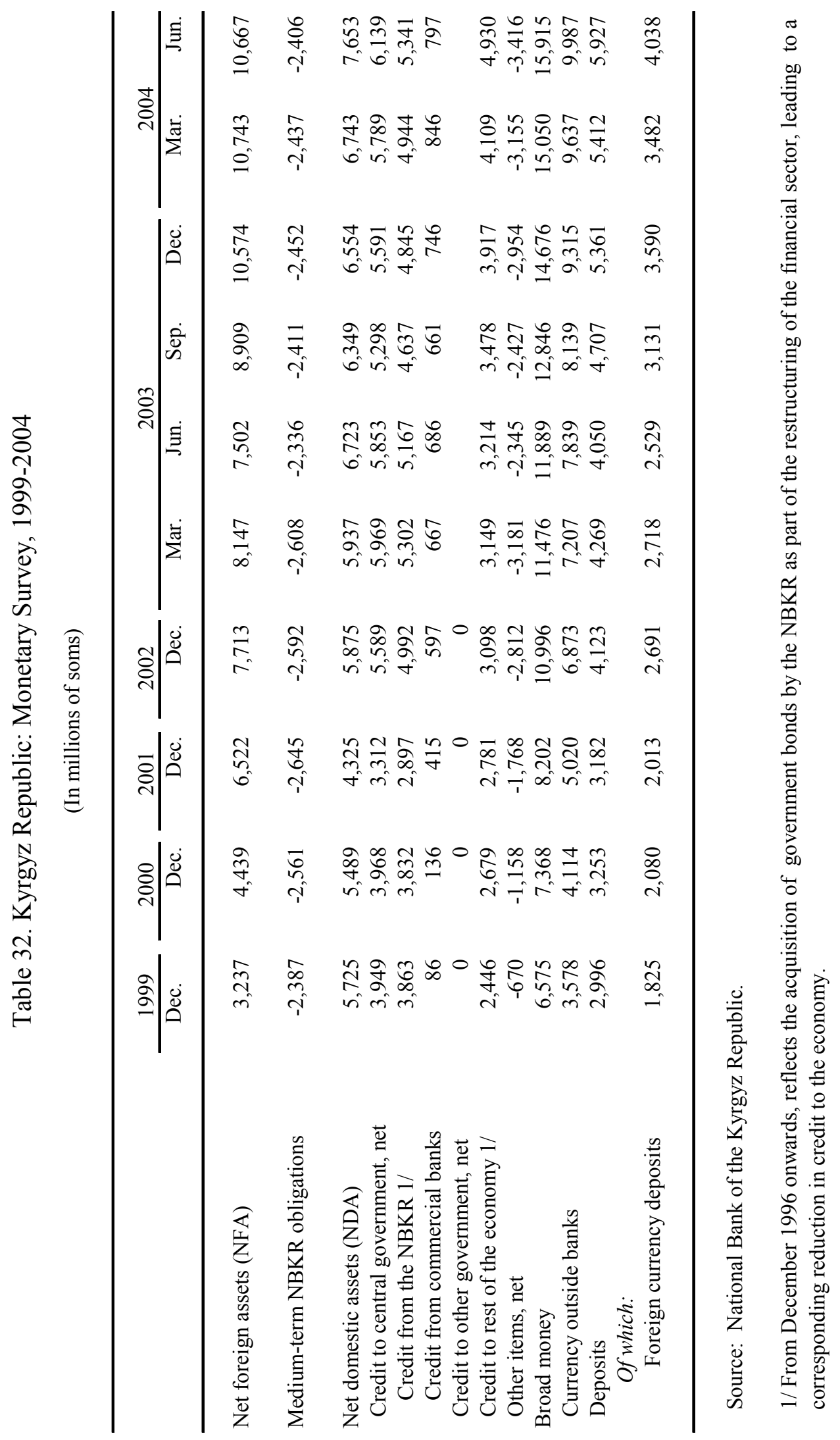


Table 33. Kyrgyz Republic: Key Central Bank Interest Rates, 1998-2004

(end of period; in percent)

\begin{tabular}{|c|c|c|c|c|c|c|c|}
\hline & Prime 1/ & $\begin{array}{l}\text { 3-month } \\
\text { Treasury Bill } \\
\text { 2/ }\end{array}$ & Lombard 3/ & Reverse repo 4/ & Repo 5/ & $\begin{array}{l}\text { Reserve } \\
\text { requirement } \\
\text { (RR) }\end{array}$ & $\begin{array}{l}\text { Penalty rate for } \\
\text { RR non- } \\
\text { compliance 6/ }\end{array}$ \\
\hline \multicolumn{8}{|l|}{1998} \\
\hline March & 24.5 & 24.5 & 29.4 & 21.5 & n.a. & 20.0 & 34.4 \\
\hline June & 50.0 & 41.5 & 60.0 & 50.0 & 60.0 & 20.0 & 100.0 \\
\hline September & 50.0 & 58.9 & 70.0 & 65.0 & n.a. & 20.0 & 140.0 \\
\hline December & 32.9 & 32.9 & 90.9 & 65.0 & n.a. & 20.0 & 138.1 \\
\hline \multicolumn{8}{|l|}{1999} \\
\hline March & 32.0 & 32.0 & 32.0 & 23.0 & n.a. & 20.0 & 90.6 \\
\hline June & 45.8 & 45.8 & 62.8 & 49.0 & n.a. & 20.0 & 199.7 \\
\hline September & 54.2 & 54.2 & 54.2 & 38.9 & 54.5 & 20.0 & 166.0 \\
\hline December & 55.1 & 55.1 & 62.4 & 56.0 & n.a. & 20.0 & 172.2 \\
\hline \multicolumn{8}{|l|}{2000} \\
\hline March & 42.1 & 42.1 & 42.1 & 39.0 & n.a. & 20.0 & 127.1 \\
\hline June & 14.0 & 15.6 & 14.0 & n.a. & n.a. & 20.0 & 58.0 \\
\hline September & 40.8 & 31.9 & 40.8 & 39.8 & n.a. & 20.0 & 117.4 \\
\hline December & 38.3 & 44.8 & 38.3 & 40.2 & n.a. & 20.0 & 120.0 \\
\hline \multicolumn{8}{|l|}{2001} \\
\hline March & 9.6 & 21.4 & 20.0 & 13.0 & n.a. & 20.0 & 23.6 \\
\hline June & 9.3 & 19.3 & 20.0 & n.a. & n.a. & 15.0 & 25.4 \\
\hline September & 9.1 & 18.6 & 15.0 & n.a. & n.a. & 10.0 & 31.7 \\
\hline December & 8.0 & 12.4 & 15.0 & n.a. & 15.0 & 10.0 & 20.5 \\
\hline \multicolumn{8}{|l|}{2002} \\
\hline March & 5.0 & 7.9 & 6.0 & 8.5 & n.a. & 10.0 & 15.4 \\
\hline June & 5.9 & 9.9 & 7.1 & 5.8 & 9.9 & 10.0 & 17.7 \\
\hline September & 6.8 & 11.7 & 8.2 & 7.0 & 7.8 & 10.0 & 20.4 \\
\hline December & 4.4 & 7.0 & 5.3 & n.a. & n.a. & 10.0 & 13.5 \\
\hline \multicolumn{8}{|l|}{2003} \\
\hline March & 4.0 & 6.0 & 4.8 & 4.5 & n.a. & 10.0 & 12.0 \\
\hline June & 4.0 & 9.5 & 4.8 & 4.5 & n.a. & 10.0 & 12.0 \\
\hline September & 4.0 & 10.0 & 4.8 & 4.3 & n.a. & 10.0 & 12.0 \\
\hline December & 4.0 & 3.8 & 4.8 & 6.1 & n.a. & 10.0 & 11.9 \\
\hline \multicolumn{8}{|l|}{2004} \\
\hline January & 4.1 & 4.1 & 4.9 & 7.2 & n.a. & 10.0 & 12.3 \\
\hline February & 4.1 & 4.6 & 4.9 & 4.8 & n.a. & 10.0 & 12.8 \\
\hline March & 5.1 & 4.9 & 6.1 & 4.5 & n.a. & 10.0 & 15.1 \\
\hline April & 5.1 & 4.9 & 6.1 & n.a. & 6.1 & 10.0 & 15.2 \\
\hline May & 4.9 & 4.7 & 5.8 & n.a. & n.a. & 10.0 & 14.6 \\
\hline June & 4.9 & 5.4 & 5.8 & 5.8 & 5.8 & 10.0 & 14.6 \\
\hline July & 4.8 & 5.9 & 5.8 & n.a. & n.a. & 10.0 & 14.4 \\
\hline August & 4.8 & 5.6 & 5.8 & n.a. & 5.8 & 10.0 & 14.5 \\
\hline
\end{tabular}

Source: National Bank of the Kyrgyz Republic.

1/ Until December 1996, NBKR credit auction rate. Since January 1997, after the abolition of credit auctions, 3-month Treasury Bill rate in the primary market. Since June 1998, set independently by NBKR Board of Directors. Since December 1998, again the 3-month Treasury Bill rate. Since June 2000, the 28-days Note of NBKR rate.

2/ Weighted average annual rate on 3-month Treasury Bills in the primary market.

3/ Rate at which the NBKR extends collateralized short-term liquidity loans to banks. Since December 1998, Lombard facility is an Overnight facility. Since March 2002, Overnight rate equal to 1.2 times the prime interest rate.

4/ Rate at which the NBKR sells government securities to commercial banks.

5/ Rate at which the NBKR purchases government securities from commercial banks.

6/ Until March 1997, penalty rate for non-compliance was 1.2 times the 3-month Treasury Bill rate in the primary market at the end of the reporting period. Between April 1997 and July 1998, penalty rate was raised to 1.4 times the Treasury Bill rate. In August 1998, penalty rate was raised to 2 times the Lombard rate, and beginning November 17, 1998, to three times the Lombard rate. As of December 1, 1998, the penalty rate was set at three times the prime interest rate at the end of the reporting period. 
Table 34. Kyrgyz Republic: Interest Rates Developments, 1998-2004 1/

(In percent)

\begin{tabular}{|c|c|c|c|c|c|c|c|c|c|c|}
\hline & & \multicolumn{2}{|c|}{ Credits 2/ } & \multicolumn{2}{|c|}{$\begin{array}{c}\text { New deposits of legal } \\
\text { entities } 3 /\end{array}$} & \multicolumn{2}{|c|}{$\begin{array}{c}\text { New deposits of } \\
\text { individual clients } 3 \text { / }\end{array}$} & \multicolumn{2}{|c|}{ Retail deposits 4/ } & \multirow{2}{*}{$\begin{array}{c}\text { 3-month } \\
\text { Treasury } \\
\text { Bills 5/ }\end{array}$} \\
\hline & & U.S. dollars & Soms & U.S. dollars & $\overline{\text { Soms }}$ & U.S. dollars & $\overline{\text { Soms }}$ & U.S. dollars & $\overline{\text { Soms }}$ & \\
\hline \multirow[t]{4}{*}{1998} & March & 37.70 & 52.08 & - & 24.06 & 15.39 & 30.17 & 5.05 & 34.20 & 24.54 \\
\hline & June & 39.46 & 55.65 & - & 32.11 & 14.09 & 27.33 & 14.89 & 27.98 & 41.53 \\
\hline & September & 46.35 & 67.77 & 17.37 & 26.66 & 14.69 & 36.61 & 12.61 & 37.55 & 58.85 \\
\hline & December & 43.61 & 73.44 & 20.98 & 35.76 & 21.50 & 66.64 & 11.52 & 64.10 & 32.54 \\
\hline \multirow[t]{4}{*}{1999} & March & 35.76 & 56.00 & 19.14 & 26.88 & 16.20 & 35.13 & 5.76 & 29.55 & 32.03 \\
\hline & June & 19.12 & 51.77 & 17.71 & 23.50 & 14.95 & 34.34 & 5.23 & 25.42 & 45.77 \\
\hline & September & 35.84 & 66.73 & 16.98 & 22.48 & 11.14 & 32.32 & 2.67 & 12.48 & 54.20 \\
\hline & December & 37.01 & 60.86 & 9.68 & 29.67 & 10.75 & 37.42 & 2.29 & 20.07 & 55.07 \\
\hline \multirow[t]{4}{*}{2000} & March & 33.98 & 56.17 & 8.89 & 27.84 & 7.78 & 33.70 & 1.70 & 16.54 & 42.11 \\
\hline & June & 37.51 & 71.21 & 8.40 & 24.81 & 7.23 & 31.53 & 0.88 & 12.62 & 15.58 \\
\hline & September & 28.33 & 52.71 & 8.88 & 19.53 & 6.64 & 15.89 & 0.75 & 4.64 & 31.89 \\
\hline & December & 27.99 & 51.90 & 4.95 & 22.98 & 6.63 & 17.16 & 1.19 & 9.72 & 44.79 \\
\hline \multirow[t]{4}{*}{2001} & March & 26.31 & 44.99 & 10.23 & 14.29 & 6.43 & 16.08 & 0.82 & 10.29 & 21.39 \\
\hline & June & 28.67 & 40.60 & 7.05 & 15.43 & 5.05 & 12.95 & 0.74 & 5.99 & 19.25 \\
\hline & September & 26.25 & 35.63 & 7.86 & 12.56 & 6.05 & 12.10 & 0.83 & 7.86 & 18.55 \\
\hline & December & 25.70 & 37.33 & 0.02 & 13.33 & 5.08 & 11.30 & 1.21 & 6.15 & 12.36 \\
\hline \multirow[t]{4}{*}{2002} & March & 24.80 & 39.92 & 4.41 & 7.90 & 4.50 & 9.65 & 0.37 & 5.92 & 7.87 \\
\hline & June & 23.82 & 36.52 & 2.04 & 2.50 & 5.13 & 8.21 & 0.45 & 3.57 & 9.89 \\
\hline & September & 23.28 & 34.76 & 0.00 & 8.10 & 4.15 & 7.61 & 0.32 & 2.80 & 11.65 \\
\hline & December & 20.15 & 24.81 & 6.17 & 5.27 & 4.73 & 7.09 & 0.27 & 2.34 & 7.01 \\
\hline \multirow[t]{4}{*}{2003} & March & 22.70 & 34.11 & 1.15 & 4.99 & 3.85 & 5.95 & 0.25 & 1.72 & 5.98 \\
\hline & June & 20.68 & 17.58 & 2.06 & 4.74 & 3.36 & 6.01 & 0.34 & 2.63 & 9.52 \\
\hline & September & 19.67 & 20.00 & 3.00 & 5.64 & 3.66 & 4.94 & 0.68 & 1.88 & 10.02 \\
\hline & December & 21.74 & 19.13 & 1.65 & 3.14 & 3.77 & 6.63 & 0.19 & 1.62 & 3.79 \\
\hline \multirow[t]{7}{*}{2004} & January & 25.67 & 26.54 & 4.49 & 4.45 & 3.20 & 5.54 & 0.24 & 1.25 & 4.12 \\
\hline & February & 17.13 & 18.14 & 3.00 & 4.69 & 4.61 & 5.20 & 0.21 & 2.10 & 4.56 \\
\hline & March & 17.70 & 31.35 & 5.97 & 4.31 & 3.61 & 5.33 & 0.35 & 2.00 & 4.80 \\
\hline & April & 21.26 & 23.01 & 3.00 & 3.82 & 4.26 & 5.08 & 0.30 & 2.28 & 4.89 \\
\hline & May & 20.88 & 16.27 & 3.00 & 6.04 & 3.88 & 5.06 & 0.16 & 1.35 & 4.68 \\
\hline & June & 21.81 & 16.73 & 3.00 & 4.89 & 3.87 & 5.77 & 0.17 & 1.66 & 5.41 \\
\hline & July & 18.99 & 20.89 & 3.00 & 4.73 & 3.64 & 6.94 & 0.14 & 1.68 & 5.94 \\
\hline
\end{tabular}

Source: National Bank of the Kyrgyz Republic.

$1 /$ Rates refer to new credits and deposits extended during the month.

2/ Weighted average annual rate on new loans granted for a period of 1-3 months to all sectors.

3/ Weighted average annual rate on new deposits for a period of 1-3 months.

4/ Weighted average annual rate on new deposits from individual clients for all periods.

5/ Weighted average annual rate on 3-month Treasury Bills in the primary market, at the end of period, for date of auction. 
Table 35. Kyrgyz Republic: Foreign Exchange Auctions and Interbank Market, 1999-2004 (In millions of U.S. dollars unless stated otherwise)

\begin{tabular}{|c|c|c|c|c|c|c|c|c|}
\hline & \multirow{2}{*}{$\begin{array}{c}\text { Volume of } \\
\text { NBKR sales to } \\
\text { Central } \\
\text { Treasury } \\
\text { (1) } \\
\end{array}$} & \multirow{2}{*}{$\begin{array}{l}\text { Volume of } \\
\text { NBKR sales to } \\
\text { private sector } \\
(2)=(3)+(4) \\
\end{array}$} & \multicolumn{2}{|c|}{ Of which: } & \multirow{2}{*}{$\begin{array}{l}\begin{array}{l}\text { Total volume } \\
\text { of NBKR sales }\end{array} \\
(5)=(1)+(2)\end{array}$} & \multirow{2}{*}{$\begin{array}{c}\text { NBKR } \\
\text { purchases in } \\
\text { interbank } \\
\text { market } \\
(6) \\
\end{array}$} & \multicolumn{2}{|c|}{ Som per U.S. dollar 1/ } \\
\hline & & & $\begin{array}{l}\text { Volume of } \\
\text { NBKR sales in } \\
\text { auctions } \\
\text { (3) }\end{array}$ & $\begin{array}{c}\text { NBKR sales in } \\
\text { interbank } \\
\text { market } \\
(4) \\
\end{array}$ & & & Average & End-of-period \\
\hline 1999 & 18.8 & 33.6 & n.a. & 33.6 & 52.4 & 10.8 & 39.2 & 45.3 \\
\hline January & 1.2 & 6.7 & n.a. & 6.7 & 7.9 & 0.0 & 30.1 & 30.4 \\
\hline February & 0.6 & 0.0 & n.a. & 0.0 & 0.6 & 4.3 & 30.7 & 33.8 \\
\hline March & 0.8 & 1.2 & n.a. & 1.2 & 2.0 & 1.2 & 32.2 & 30.4 \\
\hline April & 2.3 & 3.9 & n.a. & 3.9 & 6.2 & 0.0 & 37.0 & 38.5 \\
\hline May & 0.4 & 3.7 & n.a. & 3.7 & 4.2 & 0.0 & 41.2 & 43.2 \\
\hline June & 2.1 & 1.0 & n.a. & 1.0 & 3.1 & 0.0 & 42.2 & 41.2 \\
\hline July $2 /$ & 1.3 & 0.0 & n.a. & 0.0 & 1.3 & 0.3 & 39.8 & 40.5 \\
\hline August & 0.7 & 7.4 & n.a. & 7.4 & 8.1 & 0.0 & 41.7 & 42.5 \\
\hline September & 1.6 & 1.3 & n.a. & 1.3 & 3.0 & 0.5 & 42.8 & 42.8 \\
\hline October & 1.4 & 0.4 & n.a. & 0.4 & 1.8 & 2.6 & 42.8 & 43.0 \\
\hline November & 1.6 & 6.2 & n.a. & 6.2 & 7.8 & 0.1 & 44.4 & 45.9 \\
\hline December & 4.8 & 1.7 & n.a. & 1.7 & 6.4 & 1.9 & 45.8 & 45.3 \\
\hline 2000 & 32.4 & 34.9 & n.a. & 34.9 & 67.3 & 14.1 & 47.8 & 48.3 \\
\hline January & 0.5 & 5.3 & n.a. & 5.3 & 5.8 & 0.5 & 46.6 & 46.7 \\
\hline February & 1.4 & 2.7 & n.a. & 2.7 & 4.2 & 0.8 & 47.0 & 47.4 \\
\hline March & 1.8 & 2.2 & n.a. & 2.2 & 3.9 & 0.8 & 47.6 & 47.7 \\
\hline April & 3.9 & 1.8 & n.a. & 1.8 & 5.6 & 0.0 & 47.9 & 48.0 \\
\hline May & 2.4 & 2.3 & n.a. & 2.3 & 4.7 & 0.3 & 48.1 & 48.3 \\
\hline June & 2.7 & 0.1 & n.a. & 0.1 & 2.8 & 2.4 & 47.6 & 47.0 \\
\hline July & 1.6 & 0.9 & n.a. & 0.9 & 2.5 & 0.1 & 47.0 & 47.1 \\
\hline August & 8.7 & 4.0 & n.a. & 4.0 & 12.7 & 0.0 & 47.4 & 48.0 \\
\hline September & 0.8 & 4.1 & n.a. & 4.1 & 5.0 & 3.9 & 48.5 & 48.0 \\
\hline October & 1.3 & 3.9 & n.a. & 3.9 & 5.2 & 3.2 & 48.1 & 48.3 \\
\hline November & 4.6 & 7.0 & n.a. & 7.0 & 11.6 & 0.0 & 48.9 & 49.4 \\
\hline December & 2.6 & 0.7 & n.a. & 0.7 & 3.3 & 2.3 & 48.9 & 48.3 \\
\hline 2001 & 41.3 & 9.6 & n.a. & 9.6 & 50.9 & 18.5 & 48.4 & 47.7 \\
\hline January & 0.9 & 4.8 & n.a. & 4.8 & 5.6 & 0.0 & 48.8 & 49.1 \\
\hline February & 0.6 & 0.5 & n.a. & 0.5 & 1.1 & 0.0 & 49.1 & 49.2 \\
\hline March & 4.4 & 1.5 & n.a. & 1.5 & 5.9 & 0.0 & 49.4 & 49.5 \\
\hline April & 7.5 & 0.0 & n.a. & 0.0 & 7.5 & 0.0 & 49.6 & 49.4 \\
\hline May & 2.5 & 0.0 & n.a. & 0.0 & 2.5 & 0.2 & 49.2 & 49.2 \\
\hline June & 3.2 & 0.0 & n.a. & 0.0 & 3.2 & 1.0 & 48.3 & 48.0 \\
\hline July & 12.1 & 0.0 & n.a. & 0.0 & 12.1 & 2.5 & 47.7 & 47.7 \\
\hline August & 2.6 & 1.5 & n.a. & 1.5 & 4.1 & 0.9 & 47.9 & 47.7 \\
\hline September & 1.3 & 0.0 & n.a. & 0.0 & 1.3 & 8.5 & 47.7 & 47.7 \\
\hline October & 3.4 & 0.2 & n.a. & 0.2 & 3.7 & 3.9 & 47.8 & 47.7 \\
\hline November & 2.0 & 0.6 & n.a. & 0.6 & 2.5 & 0.0 & 47.9 & 48.0 \\
\hline December & 0.8 & 0.5 & n.a. & 0.5 & 1.3 & 1.6 & 47.9 & 47.7 \\
\hline 2002 & 6.2 & 9.2 & n.a. & 9.2 & 15.3 & 33.6 & 46.9 & 46.0 \\
\hline January & 0.1 & 2.0 & n.a. & 2.0 & 2.0 & 0.2 & 48.0 & 47.7 \\
\hline February & 0.3 & 0.0 & n.a. & 0.0 & 0.3 & 1.9 & 47.7 & 47.7 \\
\hline March & 0.2 & 1.2 & n.a. & 1.2 & 1.4 & 0.0 & 48.0 & 47.8 \\
\hline April & 0.5 & 0.6 & n.a. & 0.6 & 1.1 & 0.3 & 48.0 & 48.0 \\
\hline May & 0.7 & 0.0 & n.a. & 0.0 & 0.7 & 0.6 & 47.9 & 47.7 \\
\hline June & 0.7 & 0.0 & n.a. & 0.0 & 0.7 & 4.6 & 47.0 & 46.0 \\
\hline July & 0.3 & 1.7 & n.a. & 1.7 & 2.0 & 0.5 & 46.0 & 46.1 \\
\hline August & 0.3 & 1.5 & n.a. & 1.5 & 1.8 & 2.2 & 46.1 & 46.0 \\
\hline September & 1.0 & 0.0 & n.a. & 0.0 & 1.0 & 9.6 & 46.1 & 46.0 \\
\hline October & 0.6 & 0.0 & n.a. & 0.0 & 0.6 & 9.7 & 46.0 & 46.0 \\
\hline November & 0.8 & 0.0 & n.a. & 0.0 & 0.8 & 2.9 & 46.0 & 46.2 \\
\hline December & 0.7 & 2.2 & n.a. & 2.2 & 2.9 & 1.1 & 46.2 & 46.0 \\
\hline 2003 & 16.1 & 8.0 & n.a. & 8.0 & 24.1 & 53.0 & 43.7 & 43.6 \\
\hline January & 2.0 & 3.9 & n.a. & 3.9 & 5.9 & 0.7 & 46.5 & 46.0 \\
\hline February & 0.8 & 0.0 & n.a. & 0.0 & 0.8 & 0.0 & 46.2 & 46.2 \\
\hline March & 0.7 & 0.0 & n.a. & 0.0 & 0.7 & 2.3 & 45.6 & 45.3 \\
\hline April & 0.8 & 0.0 & n.a. & 0.0 & 0.8 & 1.6 & 44.4 & 44.0 \\
\hline May & 1.6 & 0.0 & n.a. & 0.0 & 1.6 & 2.9 & 42.9 & 42.0 \\
\hline June & 1.6 & 1.3 & n.a. & 1.3 & 2.9 & 6.5 & 41.3 & 40.8 \\
\hline July & 0.9 & 2.8 & n.a. & 2.8 & 3.7 & 0.0 & 43.0 & 43.8 \\
\hline August & 1.2 & 0.0 & n.a. & 0.0 & 1.2 & 3.4 & 42.9 & 42.5 \\
\hline September & 0.9 & 0.0 & n.a. & 0.0 & 0.9 & 8.5 & 42.9 & 42.5 \\
\hline October & 1.6 & 0.0 & n.a. & 0.0 & 1.6 & 11.6 & 41.6 & 41.4 \\
\hline November & 1.6 & 0.0 & n.a. & 0.0 & 1.6 & 8.8 & 43.1 & 43.9 \\
\hline December & 2.4 & 0.0 & n.a. & 0.0 & 2.4 & 6.7 & 44.2 & 43.6 \\
\hline 2004 & 9.1 & 1.2 & n.a. & 1.2 & 10.3 & 6.5 & 43.2 & 42.5 \\
\hline January & 1.7 & 0.0 & n.a. & 0.0 & 1.7 & 1.4 & 43.0 & 41.6 \\
\hline February & 0.4 & 0.6 & n.a. & 0.6 & 1.0 & 0.0 & 42.8 & 42.8 \\
\hline March & 0.4 & 0.0 & n.a. & 0.0 & 0.4 & 0.0 & 43.2 & 43.1 \\
\hline April & 2.3 & 0.6 & n.a. & 0.6 & 2.9 & 0.0 & 43.3 & 43.5 \\
\hline May & 2.0 & 0.0 & n.a. & 0.0 & 2.0 & 0.0 & 43.9 & 44.2 \\
\hline June & 2.3 & 0.0 & n.a. & 0.0 & 2.3 & 5.1 & 43.2 & 42.5 \\
\hline July & 1.0 & 0.0 & n.a. & 0.0 & 1.0 & 0.8 & 42.6 & 42.4 \\
\hline August & 1.1 & 0.0 & n.a. & 0.0 & 1.1 & 3.3 & 42.1 & 42.2 \\
\hline
\end{tabular}

Source: National Bank of the Kyrgyz Republic. 


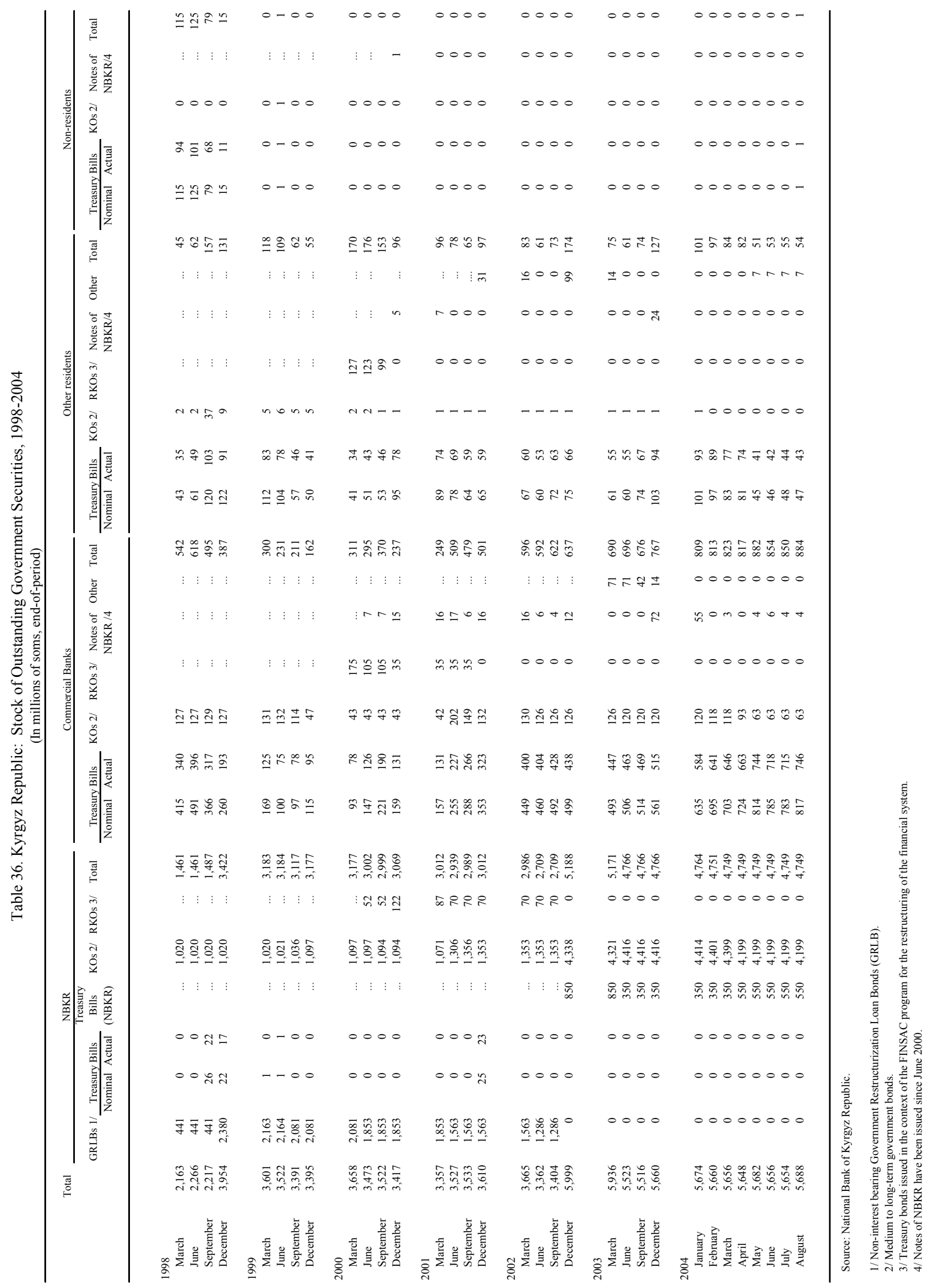




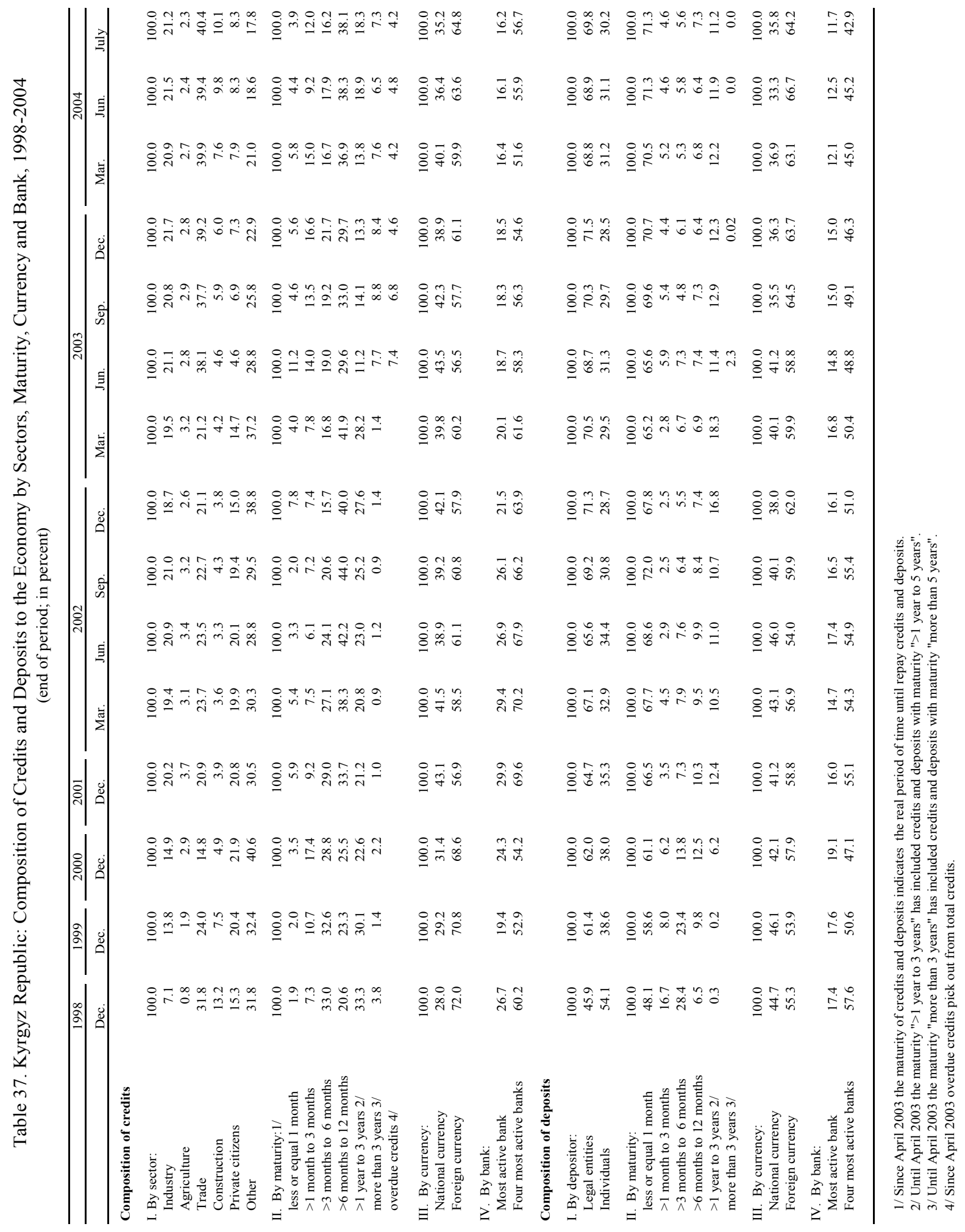




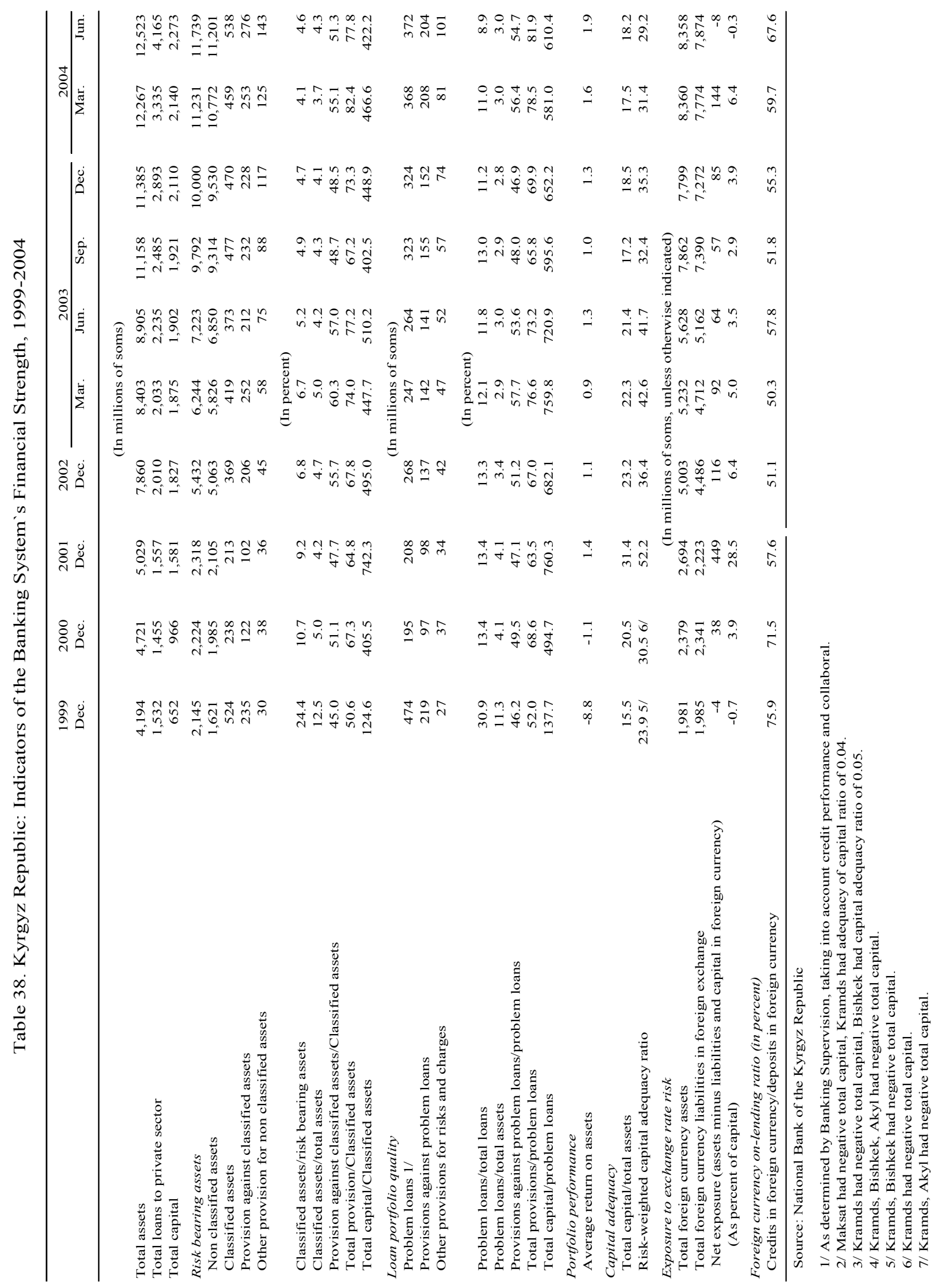


Table 39. Kyrgyz Republic: Banks with Licenses Revoked during 1996-2004

\begin{tabular}{rlr}
\hline No. & Bank & \\
& & \\
& & \\
1 & Kyrgyzelbank & $2 / 14 / 1996$ \\
2 & Agroprombank & $5 / 3 / 1996$ \\
3 & Hen-Fen Hong-Kong International Commercial Bank & $5 / 21 / 1996$ \\
4 & Orient German-Kyrgyz Bank & $5 / 21 / 1996$ \\
5 & Kyrgyz Jer Joint Bank & $12 / 17 / 1996$ \\
6 & Kyrgyzvnesh Bank & $7 / 18 / 1997$ \\
7 & Mercury Bank & $3 / 31 / 1999$ \\
8 & Maksat Bank & $4 / 9 / 1999$ \\
9 & Bishkek Bank & $2 / 24 / 2000$ \\
10 & Akyllnvest Bank & $4 / 21 / 2001$ \\
11 & Insan Bank & $5 / 17 / 2001$ \\
12 & Kyrgyz Kramds Bank 1/ & $6 / 14 / 2001$ \\
13 & Kurulush Bank & $6 / 21 / 2001$ \\
14 & Issyk-kul 2/ & $4 / 30 / 2002$ \\
15 & Kyrgyz Kramds Bank & $4 / 30 / 2002$ \\
& & \\
\hline
\end{tabular}

1/ On January 18, 2002 the bank license was returned to KyrgyzKramdsbank in accordance with the decision of the Supreme Arbitrage Court of the Kyrgyz Republic.

2/ Returned on September 25, 2002 according to court resolution 